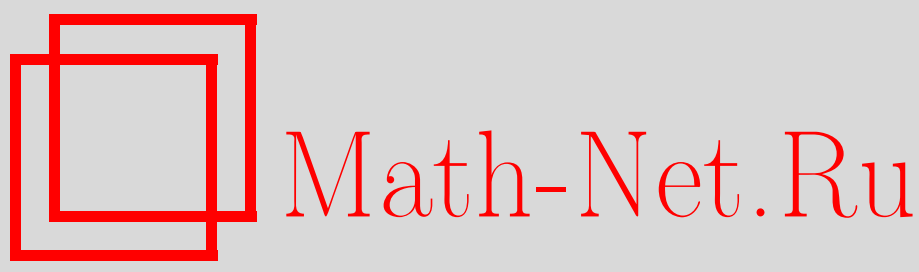

А. А. Злотник, Б. Дюкоме, Скорость стабилизации и устойчивость вязких сжимаемых баротропных симметричных течений со свободной границей для общей массовой силы, Матем. сб., 2005, том 196, номер 12, 33-84

DOI: https://doi.org/10.4213/sm1442

Использование Общероссийского математического портала Math-Net.Ru подразумевает, что вы прочитали и согласны с пользовательским соглашением

http://www . mathnet.ru/rus/agreement

Параметры загрузки:

IP: 52.87 .193 .239

26 апреля 2023 г., 13:59:09 


\section{Скорость стабилизации и устойчивость вязких сжимаемых баротропных симметричных течений со свободной границей для общей массовой силы}

Рассматриваются симметричные течения вязкой сжимаемой баротропной жидкости со свободной границей, приводимые в движение общей массовой силой $f_{S}$ (зависящей как от эйлеровой, так и от лагранжевой координат) и внешним давлением $p_{\Gamma, S}$, при общей монотонной функции состояния $p$. Охватывается случай самогравитации, возникающий в астрофизике. Сначала изучаются существование, единственность и статическая устойчивость положительных стационарных решений, дается вариационное исследование этих решений и их статической устойчивости в терминах потенциальной энергии. В астрофизическом контексте доказывается, что стационарное решение единственно и статически устойчиво при условии, что первый адиабатический показатель больше или равен $4 / 3$.

Затем в случае, когда $\omega$-предельное множество для нестационарных плотности и свободной границы содержит статически устойчивое положительное стационарное решение, выводится равномерная стабилизация к последнему и, главное, устанавливаются оценки скорости стабилизации экспоненциального типа в $L^{2}$ и $H^{1}$ для плотности и скорости при $t \rightarrow \infty$ с помощью построения новых нетривиальных функционалов Ляпунова рассматриваемой задачи. Более того, доказьвается, что статически устойчивые стационарные решения экспоненциально асимптотически устойчивы и эта нелинейная динамическая устойчивость дополнительно устойчива по отношению к малым нестационарньм возмущениям $f_{S}$ и $p_{\Gamma, S}$. Дополнительно вводится вариационное условие на стационарное решение, обеспечивающее глобальную по отношению к данньм динамическую устойчивость. Исследование вьполнено как в эйлеровых, так и в лагранжевых массовых координатах.

Библиографйл: 35 названий.

\section{§1. Введение}

Мы продолжаем наши исследования системы квазилинейных дифференциальных уравнений, описывающих симметричные течения вязкой сжимаемой баротропной жидкости, состоящей из уравнения неразрывности

$$
\rho_{t}+\frac{1}{\varkappa}(\varkappa \rho v)_{r}=0
$$

и уравнения импульса

$$
\rho\left(v_{t}+v v_{r}\right)=\left[\frac{\mu(\rho)}{\varkappa}(\varkappa v)_{r}-p(\rho)\right]_{r}+\rho f[m],
$$

Работа первого автора поддержана Российским фондом фундаменталњных исследований (гранты № № 04-01-00539, 03-01-00606 и 04-01-00619). 
в области $Q:=\left\{(r, t): r_{0}<r<R(t), t>0\right\}$, где $r_{0}>0$ - радиус (инертного) ядра, а $R(t)$ - радиус внешней свободной границы, удовлетворяюшей уравнению

$$
R^{\prime}(t)=\left.v\right|_{r=R(t)} \quad \text { при } t>0 .
$$

Здесь и ниже $\varkappa(r):=r^{k}$ с $k=0,1$ или 2 соответственно в случаях плоской, цилиндрической или сферической симметрии,

$$
m(r, t):=\int_{r_{0}}^{r} \rho(q, t) \varkappa(q) d q
$$

- лагранжева массовая координата и $f[m](r, t)=f(r, m(r, t), t)$.

Система дополняется краевыми условиями на фиксированной левой и свободной правой гранищах

$$
\left.v\right|_{r=r_{0}}=0,\left.\quad\left[\frac{\mu(\rho)}{\varkappa}(\varkappa v)_{r}-p(\rho)-2 k \mu_{1}(\rho) \frac{v}{r}\right]\right|_{r=R(t)}=-p_{\Gamma}(t) \text { при } t>0
$$

вместе с начальньми условиями

$$
\left.\rho\right|_{t=0}=\rho^{0}(r),\left.\quad v\right|_{t=0}=v^{0}(r) \text { при } r \in \Omega_{0}:=\left(r_{0}, R^{0}\right),\left.\quad R\right|_{t=0}=R^{0}>r_{0} .
$$

Искомые функции $\rho>0$ и $v$ - это плотность и скорость жидкости, $p(\rho)-$ давление $\left(s \mapsto p(s)\right.$ - соответствуюшая функция состояния), $\mu(\rho)$ и $\mu_{1}(\rho)$ - два коэффициента вязкости, $f$-массовая сила и $p_{\Gamma}-$ внешнее давление. Функции $f$ и $p_{\Gamma}$ имеют вид:

$$
f(r, \chi, t)=f_{S}(r, \chi)+\Delta f(r, \chi, t), \quad p_{\Gamma}(t)=p_{\Gamma, S}+\Delta p_{\Gamma}(t),
$$

где $f_{S}$ и $p_{\Gamma, S}$ - стационарные составляющие, а $\Delta f$ и $\Delta p_{\Gamma}$ - возмушения, стремящиеся к нулю при $t \rightarrow \infty$ в некотором слабом смысле.

Соответствующая стационарная задача состоит в нахождении пары $\left\{\rho_{S}, R_{S}\right\}$, где $\rho_{S} \geqslant 0$ - стационарная плотность и $R_{S}, r_{0}<R_{S}<\infty,-$ свободный радиус, для которых выполняется нелинейное интегро-дифференциальное уравнение

$$
p\left(\rho_{S}\right)_{r}=\rho_{S} f_{S}\left[m_{S}\right] \quad \text { с } m_{S}(r):=\int_{r_{0}}^{r} \rho_{S}(q) \varkappa(q) d q \text { в } \quad \Omega_{S}:=\left(r_{0}, R_{S}\right)
$$

при условии свободной границы и ограничении на массу

$$
\begin{gathered}
\left.p\left(\rho_{S}\right)\right|_{r=R_{S}}=p_{\Gamma, S}, \\
\int_{\Omega_{S}} \rho_{S}(q) \varkappa(q) d q=M:=\int_{\Omega_{0}} \rho^{0}(q) \varkappa(q) d q .
\end{gathered}
$$

Здесь $m_{S}$ - стационарная лагранжева массовая координата и

$$
f_{S}\left[m_{S}\right](r)=f_{S}\left(r, m_{S}(r)\right) .
$$

Прокомментируем наши основные предположения. Рассматривается массовая сила $f_{S}$, зависяшая как от эйлеровой, так и от лагранжевой координат, для того чтобы охватить разнообразные приложения, включив не только случай $f_{S}=$ $f_{S}(r)$ (или $f_{S}=f_{S}(\chi)$ ), наиболее часто изучавшийся в литературе, но и $f_{S}=$ $f_{G}(r, \chi):=-G\left(M_{0}+\chi\right) / r^{2}$ с $G>0$ и $M_{0} \geqslant 0$, здесь $f_{S}$ представляет собой 
точное выражение для гравитационной силы в астрофизическом случае (со сферической симметрией), см. [1]-[4] и более конкретно [5]-[8]. Вообще говоря, на поле сил $f_{S}$ не налагается условий малости, знакоопределенности или монотонности, хотя демонстрируется роль, которую они играют. Рассматривается также общее уравнение состояния $p=p(\rho)$ с $p^{\prime}>0$, включая известную политропную модель $p(\rho)=p_{0} \rho^{\gamma}$ с любыми $\gamma>0$ и $p_{0}>0$ (см. обсуждение соответствия этой модели астрофизике в [9]). Берутся два зависящих от плотности коэффициента вязкости $\mu=\mu(\rho)$ и $\mu=\mu_{1}(\rho)$, для того чтобы охватить более реалистичные физические ситуации. С другой стороны, мы ограничиваемся случаем $r_{0}>0$ и $p_{\Gamma, S}>0$.

В первой части данного исследования (см. [10], а также краткую версию [11]) были получены равномерные по времени оценки решения и изучены свойства его стабилизации (включая $\omega$-предельные множества для $\{\rho, R\}$ ) при $t \rightarrow \infty$ без анализа скорости стабилизации и асимптотической устойчивости.

В настоящей второй части (которая замкнута в себе) мы обращаемся к этим двум важным вопросам вместе с анализом имеюших к ним отношение свойств стационарной задачи. Опишем полученные результаты. Исследование начинается с анализа эйлеровой стационарной задачи со свободной границей (7)-(9) в 2 . Сначала представлен общий результат о сушествовании решений и его следствие, относящееся к положительным решениям, т.е. с $\rho_{S}>0$, и затем изучаются в основном такие решения. Даны также явные достаточные условия единственности. В частности, в астрофизическом контексте второе из них справедливо при достаточно больших $r_{0}$ или $p_{\Gamma, S}$ или при достаточно малых $M$. Затем определяется $c m a-$ тическая устойчивость как положительность собственных значений ассоциированной линейной второго порядка обыкновенной дифференциальной задачи на собственные значения, возникающей в результате применения метода малых возмущений. Проверяется непрерывность минимального собственного значения подобных задач по отношению к вариации области (что используется в дальнейшем) и даются достаточные условия его положительности. Выводятся также $C^{1}-$ и $H^{2}$-оценки для $\rho_{S}$ и доказывается, что статически устойчивые решения изолированы (другими словами, локально единственны) в некотором сильном смысле.

Затрагивается также вариационный аспект задачи. Статическая потенциальная энергия переписывается в виде нелинейного функционала $\mathscr{R}$ одномерного вариационного исчисления со свободным правым концом относительно функции $m_{S}$ с ограничением заданной полной массы. Проверяется, что стационарные решения, и только они, являются стационарньми точками $\mathscr{R}$. Более того, условие статической устойчивости означает положительность второй вариации $\mathscr{R}$. Конечно, результаты такого типа являются классическими в механике; в астрофизическом контексте см., например, [2]-[4].

Результаты, относящиеся к эйлеровой нестационарной задаче со свободной гранищей (1)-(6), собраны в $\S 3$. В теореме 1 кратко представлены основные используемые результаты из [10] относительно равномерных по времени оценок решения и их стабилизации. Фактически результаты дополняются: в случае, когда $\omega$-предельное множество для $\{\rho, R\}$ (которое целиком состоит из стационарных решений) содержит статически устойчивое положительное решение $\left\{\rho_{S}, R_{S}\right\}$, выводится просто равномерная стабилизация к этому решению.

Нашей основной целью является существенно более глубокое изучение этой стабилизации посредством вывода оценок скорости стабилизации в нормах $L^{2}$ и $H^{1}$. 
Это реализуется в теореме 2 , где устанавливаются оценки скорости стабилизации в $L^{2}\left(\Omega_{t}\right)$ как для $\rho-\rho_{S}$, так и для $v$, или в $H^{1}\left(\Omega_{t}\right)$ для $\rho-\rho_{S}$ и в $L^{2}\left(\Omega_{t}\right)$ для $v$ и, наконец, в $H^{1}\left(\Omega_{t}\right)$ как для $\rho-\rho_{S}$, так и для $v$. Здесь $\Omega_{t}=\left(r_{0}, R(t)\right)$. Как следствие справедлива и оценка для $R-R_{S}$. Эти оценки скорости стабилизации являются оценками экспоненциального типа. Фактическая скорость стабилизации зависит от того, с какой скоростью стремятся к нулю возмушения $\Delta f$ и $\Delta p_{\Gamma}$. Убывание решений является на самом деле экспоненциальным, если таково же убывание возмущений (в частности, если они нулевые). Чтобы вьвести эти оценки, строятся и изучаются подходящие нетривиальные функционалы Ляпунова. Следствие теоремы 2 дает $H^{2,1}(Q)$-оценку для $v$.

Более того, показьвается, что эти оценки скорости стабилизации верны для любых значений времени $t \geqslant 0$ равномерно относительно начальных данных, достаточно близких в $L^{2}$-норме к любому выбранному статически устойчивому стационарному решению $\left\{\rho_{S}, R_{S}\right\}$ при условии, что возмущения $\Delta f$ и $\Delta p_{\Gamma}$ также достаточно малы (скажем, в $L^{2}$-норме). Тем самым обосновывается, что статическая устойчивость положительных стационарных решений обеспечивает их нелинейную динамическую устойчивость (более точно, их экспоненциальную асимптотическую устойчивость), которая дополнительно устойчива по отношению к малым нестационарным возмущениям $f_{S}$ и $p_{\Gamma, S}$. Легко видеть, что фактически этот результат верен также при немонотонной $p$ при условии, что значения $\rho_{S}$ принадлежат интервалу устойчивости $p$, где $p^{\prime}>0$.

Остальная часть статьи посвящена соответствуюшему изучению лагранжевых постановок стационарной и нестационарной задач в фиксированной области, что существенно обогащает исследование в целом. В $\S 4$ рассматривается лагранжева стационарная задача в нескольких эквивалентных формах. Сначала дается иное доказательство сушествования положительных решений, установленного в $\S 2$, с помощью теоремы Шаудера о неподвижной точке и выводятся другие условия единственности. Дается также другое определение статической устойчивости по аналогии с $\S 2$. Интересно, что ассоциированные задачи на собственные значения в эйлеровых и лагранжевых координатах выглядят различными, поскольку содержат производные $f_{S}$ по разным переменным (соответственно $\chi$ и $r$ ), а краевые условия на правом конце в них также различны (соответственно третьего и второго родов). Тем не менее доказывается эквивалентность этих двух определений статической устойчивости. После записи статической потенциальной энергии как нелинейного функционала $\mathscr{P}$ относительно стационарного объема, теперь с фиксированными концами и без ограничений, сначала проверяется, что именно лагранжевы стационарные решения соответствуют стационарным точкам $\mathscr{P}$, а затем, что второе определение статической устойчивости эквивалентно положительности второй вариации $\mathscr{P}$. Отметим, что в обшем случае ни $\mathscr{R}$, ни $\mathscr{P}$ не являются выпуклыми. Далее показывается, что статически устойчивые лагранжевы решения также изолированы в некотором сильном смысле, и они идентифицируются как точки локального квадратичного минимума $\mathscr{P}$.

В заключение параграфа с использованием достоинств лагранжевой формулировки приводятся новые важные достаточные условия статической устойчивости и условия единственности стационарных решений. Для вьвода последних используется метод стрельбы. В астрофизическом контексте оба условия сводятся к следующему условию на первый адиабатический показатель: 


$$
\Gamma_{1}(s):=\frac{s p^{\prime}(s)}{p(s)} \geqslant \frac{4}{3}
$$

отметим, что показатель $4 / 3$ хорошо известен в литературе как критический. В случае $p(\rho)=p_{0} \rho^{\gamma}$, когда $\Gamma_{1} \equiv \gamma$, см. связанные (но иные) результаты в [1]-[4], [6] и $[12]-[14]$.

В $\S 5$ представлена нестационарная задача в лагранжевых массовых координатах и даны аналоги теорем 1 и 2 . В качестве основного результата в теореме 3 устанавливаются оценки скорости стабилизации в $L^{2}(0, M)$ и $H^{1}(0, M)$ (экспоненциального типа), а также результат о нелинейной динамической устойчивости. Хотя эти оценки и их доказательства сравнительно близки к соответствующим им в эйлеровых координатах, они не идентичны, и представляется важньм развивать оба подхода, поскольку фактически они дополняют друг друга. Кроме того, в последнем пункте теоремы 3 показывается, что динамическая устойчивость является глобальной по отношению к данньм при некотором более ограничительном вариационном условии на стационарное решение. В заключение рассматривается связь между оценками скорости стабилизации в эйлеровых и в лагранжевых координатах.

Краткая версия этих результатов представлена в [15], [16].

Чтобы завершить введение, дадим краткие литературные комментарии. Поскольку общий обзор был дан в [10], здесь мы процитируем только немногие существующие статьи, содержащие оценки скорости стабилизации при ненулевой $f_{S}$. Задача со свободной границей исследовалась в основном в простейшем случае плоской симметрии $(k=0)$. Случай $f_{S}=f_{S}(r),\left(f_{S}\right)_{r} \leqslant 0$, был изучен в [17] в лагранжевых массовых координатах (на основе подхода, впервые развитого для ситуации с фиксированной границей в [18]). Случай $f_{S}=f_{S}(r), f_{S} \leqslant 0$ (и $p(\rho)=$ $p_{0} \rho^{\gamma}$ ), был рассмотрен в [19] (на основе модифицированного подхода из [18], данного в [20]). В эйлеровых координатах случай $f_{S}=f_{S}(r)$ с $f_{S}(r) \leqslant 0$ в окрестности точки $r=R_{S}$ был разобран в [21]. Случай $f_{S}=f_{S}(\chi)$ при вырождении $\rho_{S}$ был рассмотрен в лагранжевых массовых координатах в [22] (отметим, что скорость стабилизации оказалась там степенного типа в отличие от экспоненциальной в предыдуших статьях), см. также более раннюю статью [23].

В общем случае $(k=0,1,2)$, включающем намного более громоздкий сферически симметричный случай $(k=2)$, результаты [17] были обобщены в [24] при $\left(f_{S} / \varkappa\right)_{r} \leqslant 0$ и $\mu_{1}=0$ (на основе ранее написанной, но позже опубликованной статьи [25] для ситуации с фиксированной границей). Разностный аналог некоторых результатов из [24] содержится в [26]. Отметим также статьи [27], [28], где при $k=2$ случай $p=p_{0} \rho^{\gamma}$ с $\gamma=1$ и $\gamma$ близким к 1 был рассмотрен в ситуации с фиксированной границей. В астрофизическом контексте задача изучалась в [29] при $p_{\Gamma}=0$ и в теплопроводном случае в [30], где в результатах о стабилизации требуются условия малости $p$ или $1 / r_{0}$; см. также [14] о некоторых локальных результатах в трехмерном случае без предположения о симметрии.

\section{§2. Эйлерова стационарная задача}

Во всей статье будем предполагать, что

$$
\begin{gathered}
p \in C\left(\overline{\mathbb{R}}^{+}\right), \quad p \text { возрастает на } \overline{\mathbb{R}}^{+}, \quad p\left(\overline{\mathbb{R}}^{+}\right)=\overline{\mathbb{R}}^{+}, \quad p^{\prime} \in C\left(\mathbb{R}^{+}\right), \\
f_{S} \in C\left(\left[r_{0}, \infty\right) \times\left[0, M_{1}\right]\right) \quad \text { при некотором } M_{1}>M .
\end{gathered}
$$


Обозначим через $p^{-1}$ функцию, обратную к $p$, и введем первообразные функции

$$
F(r, \chi):=\int_{r_{0}}^{r} f_{S}(q, \chi) d q, \quad \pi(s):=\int_{1}^{s} \frac{p^{\prime}(\zeta)}{\zeta} d \zeta, \quad P_{0}(s):=\int_{1}^{s} \frac{p(\zeta)}{\zeta^{2}} d \zeta .
$$

Положим также $P_{1}(s):=s P_{0}(s)$ при $s>0$ и $P_{1}(0):=\lim _{s \rightarrow 0^{+}} P_{1}(s)=0$ (см. [10]). $\mathrm{B}$ соответствии с [24], [10] наложим следующее общее условие на поведение $f_{S}$ при $r=\infty$ :

$$
\limsup _{r \rightarrow \infty} \frac{k+1}{r^{k+1}} \cdot \max _{0 \leqslant \chi \leqslant M} F(r, \chi) \leqslant f_{0} \quad \text { при некотором } f_{0} \geqslant 0,
$$

вместе с условием, связывающим $f_{0}$ и $p_{\Gamma, S}$, с параметром $N>1$ :

$$
N^{-1}+M f_{0} \leqslant p_{\Gamma, S} \leqslant N
$$

Для удобства читателя перечислим здесь другие основные условия на $f_{S}$ и $p$, которые будут использоваться ниже в некоторых из наших утверждений:

$$
\begin{aligned}
& \left\|\left(f_{S}\right)_{r}\right\|_{L^{1}\left(\left(r_{0}, r\right) ; C[0, M]\right)}:=\|\|\left(f_{S}\right)_{r}\left\|_{C[0, M]}\right\|_{L^{1}\left(r_{0}, r\right)} \leqslant C(r) \quad \text { при любых } r>r_{0}, \\
& \left\|\left(f_{S}\right)_{\chi}\right\|_{L^{1}\left(\left(r_{0}, \widetilde{R}_{S}\right) ; C\left[0, M_{1}\right]\right)} \leqslant N \quad \text { с } \quad \widetilde{R}_{S}:=R_{S}+\varepsilon_{S} \text { при некотором } \varepsilon_{S}>0 \text {, } \\
& \|\theta(r)[\delta]\|_{L^{1}\left(r_{0}, \widetilde{R}_{S}\right)} \rightarrow 0 \text { при } \delta \rightarrow 0^{+},
\end{aligned}
$$

где

$$
\theta(r)[\delta]:=\sup _{0<\Delta \leqslant \delta}\left\|\left(f_{S}\right)_{\chi}(r, \chi+\Delta)-\left(f_{S}\right)_{\chi}(r, \chi)\right\|_{C\left[0, M_{1}-\Delta\right]}
$$

и

$$
p^{\prime \prime} \in L_{\mathrm{loc}}^{\infty}\left(\mathbb{R}^{+}\right), \quad\left\|\left(f_{S}\right)_{r}\right\|_{L^{2}\left(\left(r_{0}, \widetilde{R}_{S}\right) ; C\left[0, M_{1}\right]\right)}+\left\|\left(f_{S}\right)_{\chi}\right\|_{L^{2}\left(\left(r_{0}, \widetilde{R}_{S}\right) ; C\left[0, M_{1}\right]\right)} \leqslant N .
$$

Будем рассматривать решения $\left\{\rho_{S}, R_{S}\right\}$ стационарной задачи $(7)-(9)$ такие, что $\rho_{S} \in C\left(\bar{\Omega}_{S}\right), \rho_{S} \geqslant 0, p_{S}:=p\left(\rho_{S}\right) \in C^{1}\left(\bar{\Omega}_{S}\right)$. Стационарное решение $\left\{\rho_{S}, R_{S}\right\}$ называется положительным, если $\left(\rho_{S}\right)_{\min }:=\min \bar{\Omega}_{S} \rho_{S}>0$. Ясно, что первое соотношение (7) вместе с (8) эквивалентны следуюшему:

$$
p\left(\rho_{S}(r)\right)=p_{\Gamma, S}-\int_{r}^{R_{S}} \rho_{S} f_{S}\left[m_{S}\right] d q \quad \text { на } \bar{\Omega}_{S} .
$$

Сформулируем сначала обший результат о сушествовании решения стационарной задачи. Пусть $K=K(N), K_{i}=K_{i}(N), i=0,1, \ldots,-$ положительные неубываюшие функции $N$, которые могут зависеть также от $p, f_{S}, M$ и т.д.

УТВЕРЖДЕНИЕ 1. Предположим, что справедливы условия (10)-(13) (последнее условие (10) не требуется). Тогда существует стационарное решение $\left\{\rho_{S}, R_{S}\right\}$ такое, что $0 \leqslant \rho_{S} \leqslant K$ на $\bar{\Omega}_{S}$ и $r_{0}+K_{0}^{-1} \leqslant R_{S} \leqslant K_{0}$. Кроме того, $\rho_{S}$ удовлетворяет уравнению (7) и оченкам $0 \leqslant \rho_{S} \leqslant K_{1}$ на $\left[r_{0}, R_{S}+\varepsilon_{S}\right]$ при некотором $\varepsilon_{S}>0$. 
ДоКАЗАТЕЛЬСТвО. При выполнении дополнительных условий

$$
p^{\prime} \in C\left(\mathbb{R}^{+}\right), \quad s p^{\prime}(s)=O(1) \text { при } s \rightarrow 0^{+}
$$

и (14) результат следует из [10; теорема 3$]$ (принимая во внимание оценки для $\rho$ и $R$ в [10; теорема 1 и замечание 1$]) ;$ см. также теорему 1 в $\S 3$ ниже. Более того, $K$ и $K_{0}$ в оценках для $\rho_{S}$ и $R_{S}$ не зависят от $p^{\prime}$ и $C(r)$.

Для того чтобы снять эти условия, сначала введем усредненные функции

$$
\begin{gathered}
p_{\delta}(s):=\frac{1}{\delta} \int_{0}^{\delta}[p(s+\alpha)-p(\alpha)] d \alpha, \quad f_{S, \delta}(r, \chi):=\frac{1}{\delta} \int_{0}^{\delta} f_{S}(r+\alpha, \chi) d \alpha \\
F_{\delta}(r, \chi):=\int_{r_{0}}^{r} f_{S, \delta}(q, \chi) d q=\frac{1}{\delta} \int_{0}^{\delta}\left[F(r+\alpha, \chi)-F\left(r_{0}+\alpha, \chi\right)\right] d \alpha
\end{gathered}
$$

при $s \geqslant 0, r \geqslant r_{0}, 0 \leqslant \chi \leqslant M$ и $0<\delta<\delta_{0}$ с достаточно мальм $\delta_{0}$. Отметим, что условие (12) влечет неравенства

$$
\limsup _{r \rightarrow \infty} \frac{k+1}{r^{k+1}} \cdot \max _{0 \leqslant \chi \leqslant M} F_{\delta}(r, \chi) \leqslant \limsup _{r \rightarrow \infty} \frac{k+1}{r^{k+1}} \cdot \frac{1}{\delta} \int_{0}^{\delta} \max _{0 \leqslant \chi \leqslant M} F(r+\alpha, \chi) d \alpha \leqslant f_{0} .
$$

Поскольку также $p_{\delta}^{\prime}(s)=[p(s+\delta)-p(s)] / \delta>0$ при $s \geqslant 0$, то все условия (10)-(12), $(13),(19)$ и (14) справедливы для $p_{\delta}, f_{S, \delta}, F_{\delta}$ в роли $p, f_{S}, F$ (последнее условие с некоторым $\left.C=C_{\delta}(r)\right)$. Следовательно, сушествуют решения $\left\{\rho_{S, \delta}, R_{S, \delta}\right\}$ стационарной задачи с $p_{\delta}, f_{S, \delta}$ в роли $p, f_{S}$ и справедливы оценки

$$
0 \leqslant \rho_{S, \delta} \leqslant K, \quad r_{0}+K_{0}^{-1} \leqslant R_{S, \delta} \leqslant K_{0} .
$$

Более того, анализируя доказательство теоремы 1 , пп. 1,2 в [10] (только в упрощенном случае $\rho^{0} \equiv$ const, $v^{0}=0, \Delta f=0$ и $\Delta p_{\Gamma}=0$ ) и используя неравенства $p(s)-p(\delta) \leqslant p_{\delta}(s) \leqslant p(s+\delta)$ при $s \geqslant 0$, находим, что $K$ и $K_{0}$ не зависят от $\delta$.

Дополнительно неравенства для $p_{\delta}$ влекут соответствующие им для обратной функции к $p_{\delta}: p^{-1}(s)-\delta \leqslant p_{\delta}^{-1}(s) \leqslant p^{-1}(s+p(\delta))$ при $s \geqslant 0$. Следовательно,

$$
\begin{gathered}
\left\|p^{-1}-p_{\delta}^{-1}\right\|_{C\left[0, s_{0}\right]} \rightarrow 0, \quad\left\|f_{S}-f_{S, \delta}\right\|_{C\left(\left[r_{0}, r\right] \times\left[0, M_{1}\right]\right)} \rightarrow 0 \\
\text { при } \delta \rightarrow 0 \quad \text { для любых } s_{0}>0, r>r_{0} .
\end{gathered}
$$

При заданном $R_{S}$ можно переписать уравнения (7)-(9) как задачу Коши для системы ОДУ (обыкновенных дифференциальных уравнений)

$$
\begin{gathered}
\frac{d p_{S}}{d r}=p^{-1}\left(p_{S}\right) f_{S}\left[m_{S}\right], \quad \frac{d m_{S}}{d r}=p^{-1}\left(p_{S}\right) \varkappa \text { на } \Omega_{S}=\left(r_{0}, R_{S}\right), \\
p_{S}\left(R_{S}\right)=p_{\Gamma, S}, \quad m_{S}\left(R_{S}\right)=M
\end{gathered}
$$

для пары функций $\left(p_{S}, m_{S}\right)$; кроме того, $m_{S}\left(r_{0}\right)=0$. Предполагается, что $p_{S}, m_{S} \in C^{1}\left(\bar{\Omega}_{S}\right), p_{S} \geqslant 0$ и $0 \leqslant m_{S} \leqslant M$ на $\left[r_{0}, R_{S}\right]$. Рассматривая эту задачу с $p_{\delta}^{-1}, f_{S, \delta}, R_{S, \delta}$ в роли $p^{-1}, f_{S}, R_{S}$, в силу указанного выше результата о сушествовании вместе с теоремой сушествования Пеано при $r>R_{S, \delta}$ получаем, что решения

$$
\left(p_{S, \delta}, m_{S, \delta}\right)=\left(p_{\delta}\left(\rho_{S, \delta}\right), \int_{r_{0}}^{r} \rho_{S, \delta} \varkappa d q\right)
$$


существуют и удовлетворяют оценкам $0 \leqslant p_{S, \delta} \leqslant K_{2}$ и $0 \leqslant m_{S, \delta} \leqslant M_{1}$ на $\left[r_{0}, R_{S, \delta}+2 \varepsilon_{S}\right]$ с $K_{2}$ и $\varepsilon_{S}>0$, не зависящими от $\delta$.

Пусть $R_{S, \delta_{n}}-$ произвольная сходящаяся последовательность с некоторым $\delta_{n} \rightarrow 0$, a $R_{S}:=\lim _{n \rightarrow \infty} R_{S, \delta_{n}}$. В силу хорошо известных свойств последовательностей решений задачи Коши (см., например, [31; теорема I.2.4]) сушествует подпоследовательность (за которой сохраняем прежнее обозначение) такая, что $\left(p_{S, \delta_{n}}, m_{S, \delta_{n}}\right)$ сходится к $\left(p_{S}, m_{S}\right)$ в $C\left[r_{0}, R_{S}+\varepsilon_{S}\right]$, где $\left(p_{S}, m_{S}\right)$ служит решением задачи Коши $(20),(21)$. Ясно, что $m_{S}\left(r_{0}\right)=0$ и $\left\{\rho_{S}, R_{S}\right\}$ с $\rho_{S}=p^{-1}\left(p_{S}\right)$ является стационарньм решением с предписанньми свойствами.

ЗАмЕчАнИЕ 1. Если выполнено условие

$$
\frac{f_{S}(r, \chi)}{\varkappa(r)} \leqslant f_{0} \text { при всех }(r, \chi) \in \bar{\Pi}_{\infty}:=\left[r_{0}, \infty\right) \times[0, M] \text { с некоторым } f_{0} \geqslant 0
$$

(более жесткое, чем (12)) вместе с (13), то любое стационарное решение $\left\{\rho_{S}, R_{S}\right\}$ положительно и, более того, $\left(\rho_{S}\right)_{\min } \geqslant p^{-1}\left(N^{-1}\right)$ (поскольку $p\left(\rho_{S}\right) \geqslant p_{\Gamma, S}-M f_{0} \geqslant$ $N^{-1}$ в силу уравнения (18) и ограничения на массу (9)). В соответствии с утверждением 1 такое решение сушествует и удовлетворяет оценке $\rho_{S} \leqslant K$ на $\bar{\Omega}_{S}$.

Замечание 1 обобшает результат о существовании из [17], [24], где $f_{S}=f_{S}(r)$. Иное его доказательство (использующее лагранжеву формулировку задачи) будет дано в $\S 4$.

ЗАмечание 2. Для положительных решений $\left\{\rho_{S}, R_{S}\right\}$ ограничение на массу (9) влечет оценку сверху

$$
R_{S} \leqslant \bar{R}_{S}\left(\left(\rho_{S}\right)_{\min }\right) \text { с } \bar{R}_{S}(\alpha):=\left(r_{0}^{k+1}+(k+1) \frac{M}{\alpha}\right)^{1 /(k+1)} .
$$

Кроме того, аналогично [10; замечание 10$] \rho_{S} \in C^{1}\left(\bar{\Omega}_{S}\right)$ при условии, что $p^{\prime}>0$, поскольку $\rho_{S}=p^{-1}\left(p_{S}\right)$ и

$$
\left(\rho_{S}\right)_{r}=\frac{1}{p^{\prime}\left(\rho_{S}\right)} \rho_{S} f_{S}\left[m_{S}\right]
$$

Дадим теперь некоторые достаточные условия единственности стационарного решения. Положим $\rho_{\Gamma, S}:=p^{-1}\left(p_{\Gamma, S}\right)$.

УТВЕРЖДЕНИЕ 2. 1) Если $f_{S} \leqslant 0$ u $f_{S}(r, \chi)$ не убъвает по $\chi \in[0, M]$ при любих $r \in\left[r_{0}, \bar{R}_{S}\left(\rho_{\Gamma, S}\right)\right]$, то стационарная задача имеет единственное решение.

2) Пусть $f_{S}$ удовлетворяет условиям $f_{S} \leqslant 0 u$

$$
\begin{gathered}
f_{S}(r, \widehat{\chi})-f_{S}(r, \chi) \geqslant-\psi(r)(\widehat{\chi}-\chi) \\
\text { при всех } 0 \leqslant \chi<\widehat{\chi} \leqslant M, \quad r_{0} \leqslant r \leqslant \bar{R}_{S}\left(\rho_{\Gamma, S}\right)
\end{gathered}
$$

c некоторой функиией $\psi \in L^{1}\left(r_{0}, r\right)$ при всех $r>r_{0}, \psi \geqslant 0$, такой, что

$$
\frac{M}{C^{(1)}\left(\rho_{\Gamma, S}\right)} \int_{r_{0}}^{\bar{R}_{S}\left(\rho_{\Gamma, S}\right)} \psi d r \leqslant 1,
$$

где $C^{(1)}\left(\rho_{\Gamma, S}\right):=\inf _{s \geqslant \rho_{\Gamma, S}} p^{\prime}(s)>0$. Тогда стационарная задача имеет единственное решение. 
ДокАЗАТЕЛЬСТво. При $\left(\rho_{S}\right)_{\min }>0$ стационарное уравнение (7) можно переписать в хорошо известной форме

$$
\left[\pi\left(\rho_{S}\right)\right]_{r}=f_{S}\left[m_{S}\right] \text { в } \Omega_{S}=\left(r_{0}, R_{S}\right) .
$$

При $f_{S} \leqslant 0$ функции $p\left(\rho_{S}\right)$ и $\rho_{S}$ не возрастают на $\left[r_{0}, R_{S}\right]$, поэтому $\left(\rho_{S}\right)_{\min }=$ $\rho_{\Gamma, S}>0$ и $R_{S} \leqslant \bar{R}_{S}\left(\rho_{\Gamma, S}\right)$.

Предположим, что сушествуют два различных решения $\left\{\rho_{S}, R_{S}\right\}$ и $\left\{\widehat{\rho}_{S}, \widehat{R}_{S}\right\}$, скажем, с $R_{S} \leqslant \widehat{R}_{S}$ и $\rho_{S} \not \equiv \widehat{\rho}_{S}$ на $\bar{\Omega}_{S}$ (приняв во внимание (9)). Ясно, что

$$
\left(\pi\left(\rho_{S}\right)-\pi\left(\widehat{\rho}_{S}\right)\right)_{r}=f_{S}\left[m_{S}\right]-f_{S}\left[\widehat{m}_{S}\right] \text { в } \Omega_{S}
$$

с $\widehat{m}_{S}:=\int_{r_{0}}^{r} \widehat{\rho}_{S} \varkappa d q$. Умножив это равенство на $-\left(m_{S}-\widehat{m}_{S}\right)$, проинтегрировав по $\Omega_{S}$ и воспользовавшись (8) и $(9)$, получим равенство

$$
\mathscr{B}_{R_{S}}\left[\rho_{S}, \widehat{\rho}_{S}\right]=0
$$

с формой

$$
\begin{aligned}
\mathscr{B}_{R_{S}}\left[\rho_{S}, \widehat{\rho}_{S}\right]:= & \int_{\Omega_{S}}\left(\pi\left(\rho_{S}\right)-\pi\left(\widehat{\rho}_{S}\right)\right)\left(\rho_{S}-\widehat{\rho}_{S}\right) \varkappa d r \\
& -\left[\pi\left(\rho_{\Gamma, S}\right)-\pi\left(\widehat{\rho}_{S}\left(R_{S}\right)\right)\right]\left(M-\widehat{m}_{S}\left(R_{S}\right)\right) \\
& +\int_{\Omega_{S}}\left(f_{S}\left[m_{S}\right]-f_{S}\left[\widehat{m}_{S}\right]\right)\left(m_{S}-\widehat{m}_{S}\right) d r=: S_{1}+S_{2}+S_{3} .
\end{aligned}
$$

В силу соотношений $(26),(8)$ и $(9)$, примененных к $\left\{\widehat{\rho}_{S}, \widehat{R}_{S}\right\}$, и условия $f_{S} \leqslant 0$ имеем

$$
S_{2}=-\int_{R_{S}}^{\widehat{R}_{S}} f_{S}\left[\widehat{m}_{S}\right] d r \int_{R_{S}}^{\widehat{R}_{S}} \widehat{\rho}_{S} \varkappa d r \geqslant 0
$$

В случае $f_{S}(r, \chi)$, не убываюшей по $\chi$, имеем $S_{3} \geqslant 0$ и поэтому $S_{1} \leqslant 0$ в силу (27). Поскольку $\pi$ возрастает на $\mathbb{R}^{+}$, то это означает, что $\rho_{S}=\widehat{\rho}_{S}$ на $\bar{\Omega}_{S}$ и приводит к противоречию.

В случае, когда вьполнены условия п. 2), запишем соотношения

$$
\begin{gathered}
S_{1}=\int_{\Omega_{S}} \int_{0}^{1} \frac{p^{\prime}\left(s_{\alpha}\right)}{s_{\alpha}} d \alpha\left(\rho_{S}-\widehat{\rho}_{S}\right)^{2} \varkappa d r \geqslant C^{(1)}\left(\rho_{\Gamma, S}\right) \bar{S}_{1} \\
\text { с } \bar{S}_{1}:=\int_{\Omega_{S}} \int_{0}^{1} \frac{d \alpha}{s_{\alpha}}\left(\rho_{S}-\widehat{\rho}_{S}\right)^{2} \varkappa d r,
\end{gathered}
$$

где $s_{\alpha}:=(1-\alpha) \widehat{\rho}_{S}+\alpha \rho_{S}$. Условие (24) влечет оценку снизу

$$
S_{3} \geqslant-\int_{\Omega_{S}} \psi\left(m_{S}-\widehat{m}_{S}\right)^{2} d r \geqslant-\int_{\Omega_{S}} \psi d r\left\|m_{S}-\widehat{m}_{S}\right\|_{C\left(\bar{\Omega}_{S}\right)}^{2}
$$

Далее, имеем

$$
\left\|m_{S}-\widehat{m}_{S}\right\|_{C\left(\bar{\Omega}_{S}\right)}^{2} \leqslant\left(\int_{\Omega_{S}}\left|\rho_{S}-\widehat{\rho}_{S}\right| \varkappa d r\right)^{2} \leqslant \int_{\Omega_{S}}\left(\int_{0}^{1} \frac{d \alpha}{s_{\alpha}}\right)^{-1} \varkappa d r \cdot \bar{S}_{1} .
$$


В силу неравенства Коши-Шварца

$$
1 \leqslant \int_{0}^{1} s_{\alpha}(r) d \alpha \int_{0}^{1} \frac{d \alpha}{s_{\alpha}(r)} \text { на } \bar{\Omega}_{S},
$$

более того, указанное неравенство является строгим в любой точке $r \in \bar{\Omega}_{S}$, где $\rho_{S}(r) \neq \widehat{\rho}_{S}(r)$. Поэтому в силу предположения $\rho_{S}(r) \not \equiv \hat{\rho}_{S}(r)$ на $\bar{\Omega}_{S}$ находим

$$
\begin{aligned}
\int_{\Omega_{S}}\left(\int_{0}^{1} \frac{d \alpha}{s_{\alpha}}\right)^{-1} \varkappa d r & <\int_{\Omega_{S}} \int_{0}^{1} s_{\alpha} d \alpha \varkappa d r \\
& =\int_{0}^{1}\left[(1-\alpha) \int_{\Omega_{S}} \widehat{\rho}_{S} \varkappa d r+\alpha \int_{\Omega_{S}} \rho_{S} \varkappa d r\right] d \alpha \leqslant M .
\end{aligned}
$$

В итоге соотношения (27)-(32) приводят к неравенству

$$
\bar{S}_{1}\left(C^{(1)}\left(\rho_{\Gamma, S}\right)-M \int_{r_{0}}^{R_{S}} \psi d r\right)<0
$$

с $\bar{S}_{1}>0$, которое противоречит условию (25).

Пункт 1) утверждения 2 обобщает результат о единственности из [32], данный в случае $f_{S}=f_{S}(r)$ и $k=0$. Фактически этот пункт справедлив без сушествования $p^{\prime}$ (если в доказательстве переопределить $\pi$ в соответствии с [18]).

$\mathrm{B}$ п. 2) если $p^{\prime}$ является не убываюшей на $\mathbb{R}^{+}$(например, если $p(s)=p_{0} s^{\gamma}$ с $\gamma \geqslant 1)$, то $C^{(1)}\left(\rho_{\Gamma, S}\right)=p^{\prime}\left(\rho_{\Gamma, S}\right)$. Условие $(25)$ справедливо при достаточно малом $M \leqslant \bar{M}\left(r_{0}, \rho_{\Gamma, S}\right)$ или при достаточно большом $\rho_{\Gamma, S} \geqslant \underline{\rho}_{\Gamma, S}\left(r_{0}, M\right)$. Кроме того, если дополнительно $\psi \in L^{1}(1, \infty)$, то это условие выполнено также при достаточно большом $r_{0} \geqslant \underline{r}_{0}\left(M, \rho_{\Gamma, S}\right)$.

В случае $f_{S}=f_{G}, k=2$ условие (25) принимает вид

$$
\frac{G M}{r_{0} C^{(1)}\left(\rho_{\Gamma, S}\right)}\left(1-\frac{r_{0}}{\bar{R}_{S}\left(\rho_{\Gamma, S}\right)}\right) \leqslant 1
$$

и следует из

$$
\frac{G M}{r_{0} C^{(1)}\left(\rho_{\Gamma, S}\right)} \leqslant 1
$$

(cp. c [30]).

Отметим, что даже в весьма специальном изотермическом случае $p(s)=p_{0} s$ и при $f_{S}=f_{S}(r)$ стационарная задача может иметь конечное, счетное или континуальное множество положительных стационарных решений [33].

Обратимся к проблеме статической устойчивости. Следуя методу малых возмущений, мы можем заменить положительное решение $\left\{\rho_{S}, R_{S}\right\}$ на $\{\rho, R\}=\left\{\rho_{S}+\Delta \rho\right.$, $\left.R_{S}+\Delta R\right\}$ с малым $\{\Delta \rho, \Delta R\}$ и линеаризовать оператор задачи $(26),(8),(9)$ с точностью до членов второго порядка малости по отношению к $\{\Delta \rho, \Delta R\}$ :

$$
\begin{aligned}
{[\pi(\rho)]_{r}-f_{S}[m] } & =\left[\frac{p^{\prime}\left(\rho_{S}\right)}{\rho_{S}} \Delta \rho\right]_{r}-\left(f_{S}\right)_{\chi}\left[m_{S}\right] \Delta m+\cdots \quad \text { в } \Omega_{S}, \\
p(\rho(R))-p_{\Gamma, S} & =p^{\prime}\left(\rho_{S}\left(R_{S}\right)\right)\left(\left(\rho_{S}\right)_{r}\left(R_{S}\right) \Delta R+\Delta \rho\left(R_{S}\right)\right)+\cdots, \\
m(R)-M & =\left(\rho_{S} \varkappa\right)\left(R_{S}\right) \Delta R+\Delta m\left(R_{S}\right)+\cdots,
\end{aligned}
$$


где $\Delta m(r):=\int_{r_{0}}^{r}(\Delta \rho) \varkappa d q$. В силу уравнения $(7)$ при $r=R_{S}$ имеем

$$
p^{\prime}\left(\rho_{S}\left(R_{S}\right)\right)\left(\rho_{S}\right)_{r}\left(R_{S}\right)=\rho_{S}\left(R_{S}\right) f_{S}\left[m_{S}\right]\left(R_{S}\right) .
$$

Поскольку $\Delta \rho=\varkappa^{-1}(\Delta m)_{r}$ и $\Delta R=-\left(\Delta m /\left(\rho_{S} \varkappa\right)\right)\left(R_{S}\right)$, то можно исключить $\Delta \rho$ и $\Delta R$ и затем рассмотреть ассоциированную задачу на собственные значения для линеаризованного оператора

$$
\begin{gathered}
-\left[\frac{p^{\prime}\left(\rho_{S}\right)}{\varkappa \rho_{S}}(\Delta m)_{r}\right]_{r}+\left(f_{S}\right)_{\chi}\left[m_{S}\right] \Delta m=\lambda a_{0} \Delta m \quad \text { в } \Omega_{S}, \\
\Delta m\left(r_{0}\right)=0,\left.\quad\left\{\frac{p^{\prime}\left(\rho_{S}\right)}{\varkappa \rho_{S}}(\Delta m)_{r}-\frac{f_{S}\left[m_{S}\right]}{\varkappa \rho_{S}} \Delta m\right\}\right|_{r=R_{S}}=0 .
\end{gathered}
$$

Здесь предполагается, что выполнено условие $(15)$ и $a_{0} \in C\left(\bar{\Omega}_{S}\right)$ - некоторая функ-

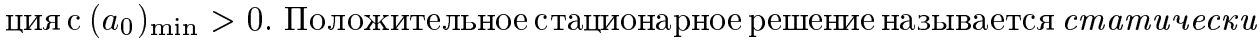
устойчивым, если

$$
\lambda_{\min }\left\{\rho_{S}, R_{S}\right\}>0
$$

где $\lambda_{\min }\left\{\rho_{S}, R_{S}\right\}$ - минимальное собственное значение этой самосопряженной задачи на собственные значения для ОДУ второго порядка. Это определение не зависит от выбора $a_{0}$.

ЗАмЕчАНИЕ 3 . В частном случае, когда $f_{S}=f_{S}(r)$, вопрос о статической устойчивости решается просто. Выбрав $a_{0}:=\rho_{S} \varkappa / p^{\prime}\left(\rho_{S}\right)$ и перейдя к новой переменной $z(r):=\int_{r_{0}}^{r} a_{0} d q$, легко преобразовать задачу на собственные значения (33), (34) к аналогичной простейшей с постоянными коэффициентами

$$
-y_{z z}=\lambda y \quad \text { на }\left(0, z_{S}\right), \quad y(0)=0, \quad y_{z}\left(z_{S}\right)-\frac{1}{\rho_{\Gamma, S}} \frac{f_{S}}{\varkappa}\left(R_{S}\right) y\left(z_{S}\right)=0,
$$

где $z_{S}=z\left(R_{S}\right)$. Последняя задача решается явно и удовлетворяет условию (35) тогда и только тогда, когда

$$
\frac{1}{\rho_{\Gamma, S}} \frac{f_{S}}{\varkappa}\left(R_{S}\right) z_{S}=\frac{f_{S}\left(R_{S}\right)}{\rho_{\Gamma, S} \varkappa\left(R_{S}\right)} \int_{\Omega_{S}} \frac{\rho_{S}}{p^{\prime}\left(\rho_{S}\right)} \varkappa d r<1
$$

Это условие очевидным образом выполнено при $f_{S}(r) \leqslant 0[19]$ или $f_{S}\left(R_{S}\right) \leqslant 0[21]$.

В дальнейшем мы будет опираться на один вспомогательный результат. Рассмотрим самосопряженную задачу на собственные значения для ОДУ второго порядка

$$
\begin{aligned}
& -\left(a w_{r}\right)_{r}+b w=\lambda w \quad \text { на }\left(r_{0}, R\right), \\
& w\left(r_{0}\right)=0, \quad\left(a w_{r}+d w\right)(R)=0,
\end{aligned}
$$

с параметром $R \in\left(r_{0}, \bar{R}\right]$ и коэффициентами $a \in L^{\infty}\left(r_{0}, \bar{R}\right)$ с $0<\delta_{0} \leqslant a$ на $\left(r_{0}, \bar{R}\right)$, $b \in L^{1}\left(r_{0}, \bar{R}\right)$ и $d \in C\left(\left(r_{0}, \bar{R}\right]\right)$. Пусть $\lambda_{\min }(R)$ - минимальное собственное значение этой задачи (которая, конечно, должна пониматься в обобщенном смысле).

УТВЕРЖДЕНИЕ 3. Имеет место свойство $\lambda_{\min }(R) \in C\left(\left(r_{0}, \bar{R}\right]\right)$. 
ДокАЗАТЕЛЬСТво. Будем краткими, поскольку результат представляет собой упражнение по спектральной теории самосопряженных эллиптических операторов. Для $w \in \widetilde{H}^{1}\left(r_{0}, R\right):=\left\{\varphi \in H^{1}\left(r_{0}, R\right): \varphi\left(r_{0}\right)=0\right\}$ введем ассоциированные с введенной задачей энергетический функционал и отношение Рэлея:

$$
\begin{aligned}
& \mathscr{J}_{R}[w]:=\int_{r_{0}}^{R}\left(a w_{r}^{2}+b w^{2}\right) d r+d(R) w^{2}(R), \\
& \left.\mathscr{R}_{R}[w]:=\mathscr{J}_{R}[w]\left(\int_{r_{0}}^{R} w^{2} d r\right)^{-1} \text { (при } w \neq \equiv 0\right) .
\end{aligned}
$$

Пусть $r_{\varepsilon_{0}}:=r_{0}+\varepsilon_{0} \leqslant R \leqslant \bar{R}$ при любом фиксированном $\varepsilon_{0} \in\left(0, \bar{R}-r_{0}\right)$. Заметим, что

$$
\begin{array}{r}
\left|\int_{r_{0}}^{R} b w^{2} d r+d(R) w^{2}(R)\right| \leqslant\left(\|b\|_{L^{1}\left(r_{0}, \bar{R}\right)}+\|d\|_{C\left[r_{\varepsilon_{0}}, \bar{R}\right]}\right)\|w\|_{C\left[r_{0}, R\right]}^{2} \\
\leqslant \varepsilon\left\|w_{r}\right\|_{\left(r_{0}, R\right)}^{2}+\varepsilon^{-1} C_{0 \varepsilon_{0}}\|w\|_{\left(r_{0}, R\right)}^{2} \quad \text { при всех } w \in \widetilde{H}^{1}\left(r_{0}, R\right)
\end{array}
$$

с любым $\varepsilon>0$ (здесь и далее в доказательствах примем сокращение $\|\cdot\|_{G}=$ $\left.\|\cdot\|_{L^{2}(G)}\right)$. Воспользовавшись этой оценкой в сочетании с формулой Рэлея

$$
\lambda_{\min }(R)=\min _{w \in \widetilde{H}^{1}\left(r_{0}, R\right)} \mathscr{R}_{R}[w]
$$

сначала получим, что

$$
\sup _{\left[r_{\varepsilon_{0}}, \bar{R}\right]}\left|\lambda_{\min }(R)\right| \leqslant C_{1 \varepsilon_{0}} .
$$

Пусть $w^{(R)} \in \widetilde{H}^{1}\left(r_{0}, R\right)$ с $\left\|w^{(R)}\right\|_{\left(r_{0}, R\right)}=1$ - собственная функция, отвечающая $\lambda_{\min }(R)$. Тогда $\mathscr{J}_{R}\left[w^{(R)}\right]=\lambda_{\min }(R)$. Эта формула вместе с (38) и (39) влечет оценку

$$
\sup _{\left[r_{\varepsilon_{0}}, \bar{R}\right]}\left\|w^{(R)}\right\|_{H^{1}\left(r_{0}, R\right)} \leqslant C_{2 \varepsilon_{0}} .
$$

Пусть теперь $r_{\varepsilon_{0}} \leqslant R_{1}<R_{2} \leqslant \bar{R}$. Применив формулу Рэлея в сочетании с условиями на коэффициенты $a, b, d$ и оценкой (40), получим

$$
\lambda_{\min }\left(R_{2}\right) \leqslant \mathscr{R}_{R_{2}}\left[\widetilde{w}^{\left(R_{1}\right)}\right]=\mathscr{R}_{R_{1}}\left[\widetilde{w}^{\left(R_{1}\right)}\right]+\alpha\left(R_{2}-R_{1}\right)=\lambda_{\min }\left(R_{1}\right)+\alpha\left(R_{2}-R_{1}\right),
$$
где $\widetilde{w}^{\left(R_{1}\right)}$ - продолжение $w^{\left(R_{1}\right)}$ постоянной $w^{\left(R_{1}\right)}\left(R_{1}\right)$ на $r>R_{1}$, а $\alpha$ - некоторая функция такая, что $\alpha(z) \rightarrow 0$ при $z \rightarrow 0^{+}$. Аналогично

$$
\lambda_{\min }\left(R_{1}\right) \leqslant \mathscr{R}_{R_{1}}\left[w^{\left(R_{2}\right)}\right]=\mathscr{R}_{R_{2}}\left[w^{\left(R_{2}\right)}\right]+\alpha\left(R_{2}-R_{1}\right)=\lambda_{\min }\left(R_{2}\right)+\alpha\left(R_{2}-R_{1}\right) .
$$

Поэтому $\left|\lambda_{\min }\left(R_{2}\right)-\lambda_{\min }\left(R_{1}\right)\right| \leqslant \alpha\left(R_{2}-R_{1}\right)$.

ЗАмечАние 4 . Напомним, что в случае $\lambda_{\min }(R)>0$ справедливо следующее неравенство:

$$
\begin{gathered}
\mathscr{J}_{R}[w] \geqslant C_{1}\left\|w_{r}\right\|_{L^{2}\left(r_{0}, R\right)}^{2} \quad \text { при всех } w \in \widetilde{H}^{1}\left(r_{0}, R\right) \\
\text { с } C_{1}:=\frac{\delta_{0}}{2}\left(1+\frac{2}{\delta_{0}} \frac{C_{0} \varepsilon_{0}}{\lambda_{\min }(R)}\right)^{-1}
\end{gathered}
$$

(которое следует из $\mathscr{J}_{R}[w] \geqslant \lambda_{\min }(R)\|w\|_{L^{2}\left(r_{0}, R\right)}^{2}$ и (38)).

Напомним также, что $w^{(R)} \in C^{1}\left(\bar{\Omega}_{S}\right)$ при условии, что $a \in C\left(\bar{\Omega}_{S}\right)$; более того, $w^{(R)} \in C^{2}\left(\bar{\Omega}_{S}\right)$ при условии, что $a \in C^{1}\left(\bar{\Omega}_{S}\right)$ и $b \in C\left(\bar{\Omega}_{S}\right)$. 
ЗАмечание 5 . Ясно, что для всех $w \in \widetilde{H}^{1}\left(r_{0}, R\right)$

$$
\|w\|_{C\left[r_{0}, R\right]}^{2} \leqslant\left(\int_{r_{0}}^{R}\left|w_{r}\right| d r\right)^{2} \leqslant\left\|\frac{1}{a}\right\|_{L^{1}\left(r_{0}, R\right)}\left\|a w_{r}^{2}\right\|_{L^{1}\left(r_{0}, R\right)} .
$$

Поэтому $\lambda_{\min }(R)>0\left(\right.$ так как $\left.\mathscr{J}_{R}[w] \geqslant\left(1-\alpha_{0}\right)\left\|a w_{r}^{2}\right\|_{L^{1}\left(r_{0}, R\right)}\right)$ при условии, что

$$
\alpha_{0}:=\left(\int_{r_{0}}^{R} b_{-}(r) d r+d_{-}(R)\right) \int_{r_{0}}^{R} \frac{d r}{a(r)}<1 ;
$$

здесь и ниже $\varphi_{-}=\max \{-\varphi, 0\}$ и $\varphi_{+}=\max \{\varphi, 0\}$.

Более того, заметим, что оба неравенства в (41) фактически являются равенствами, если и только если

$$
\frac{w(r)}{w_{0}(r)} \equiv \text { const } \quad \text { на }\left(r_{0}, R\right) \text { с } w_{0}(r)=\int_{r_{0}}^{r} \frac{d q}{a(q)} .
$$

Далее, $w_{0}$ является собственной функцией, отвечающей $\lambda=0$ (как следствие $\left.\mathscr{J}_{R}\left[w_{0}\right]=0\right)$, если и только если

$$
b=0 \text { на }\left(r_{0}, R\right), \quad d(R) \int_{r_{0}}^{R} \frac{d r}{a(r)}=-1 .
$$

Таким образом, в случае, когда $\alpha_{0}=1$ и не выполнено по крайней мере одно из условий $(42)$, опять-таки $\lambda_{\min }(R)>0$. С другой стороны, $\lambda_{\min }(R) \leqslant 0$ при условии, что

$$
w_{0}(R) \mathscr{J}_{R}\left[\bar{w}_{0}\right]=1+\left(\int_{r_{0}}^{R} b \bar{w}_{0}^{2} d r+d(R)\right) \int_{r_{0}}^{R} \frac{d r}{a(r)} \leqslant 0 \quad \text { c } \quad \bar{w}_{0}:=\frac{w_{0}}{w_{0}(R)} .
$$

В связи с нашей исходной задачей рассмотрим случай

$$
a=\frac{p^{\prime}\left(\rho_{S}\right)}{\varkappa \rho_{S}}, \quad b=\left(f_{S}\right)_{\chi}\left[m_{S}\right], \quad d=-\frac{f_{S}}{\varkappa \rho_{S}}\left(R_{S}, M\right)
$$

и $a_{0} \equiv 1$. Как следствие

$$
\lambda_{\min }(R)=\lambda_{\min }\left\{\rho_{S}, R_{S}\right\}(R), \quad \lambda_{\min }\left\{\rho_{S}, R_{S}\right\}=\lambda_{\min }\left\{\rho_{S}, R_{S}\right\}\left(R_{S}\right)
$$

И

$$
\begin{aligned}
\mathscr{J}_{R}[w]:= & \mathscr{J}_{R}\left\{\rho_{S}, R_{S}\right\}[w]=\int_{r_{0}}^{R}\left(\frac{p^{\prime}\left(\rho_{S}\right)}{\varkappa \rho_{S}} w_{r}^{2}+\left(f_{S}\right)_{\chi}\left[m_{S}\right] w^{2}\right) d r \\
& -\frac{f_{S}}{\varkappa \rho_{S}}\left(R_{S}, M\right) w^{2}(R) .
\end{aligned}
$$

Теперь представим оценки для $\rho_{S}$ и докажем, что статически устойчивые решения являются изолированными в некотором сильном смысле, что будет сушественно в следуюшем параграфее. 
УТВЕРЖДЕНИЕ 4 . Пусть $p^{\prime}>0$ на $\mathbb{R}^{+} u\left\{\rho_{S}, R_{S}\right\}$ - любое стационарное решение такое, что $\left(\rho_{S}\right)_{\min } \geqslant \delta_{S}>0$ при некотором $\delta_{S}>0$. Тогда справедливы следующие утверждения.

1) Имеет место свойство $\rho_{S} \in C^{1}\left(\bar{\Omega}_{S}\right)$ и справедлива оченка

$$
\left\|\rho_{S}\right\|_{C^{1}\left(\bar{\Omega}_{S}\right)} \leqslant K
$$

Более того, $\rho_{S}$ может бъть продолжена на $\left[r_{0}, \widetilde{R}_{S}\right], \widetilde{R}_{S}:=R_{S}+\varepsilon_{S}$ при некотором $\varepsilon_{S}>0$, так, что будут выполнятвся оченки (43), (7) и $\rho_{S} \geqslant \delta_{S} / 2$.

Величины $K$ и $\varepsilon_{S}$ в этом пункте и $\left.n .3\right)$ ниже зависят от $\delta_{S}$.

2) При выполнении условия (15) не существует решения $\left\{\widehat{\rho}_{S}, \widehat{R}_{S}\right\}$ такого, что $\widehat{R}_{S}=R_{S}$ и $\widehat{\rho}_{S} \not \equiv \rho_{S}$. Более того, $\rho_{S}$ однозначно определена на $\left[R_{S}, \widetilde{R}_{S}\right]$.

3) При выполнении условий (17) справедлива оценка $\left\|\left(\rho_{S}\right)_{r r}\right\|_{L^{2}\left(r_{0}, \widetilde{R}_{S}\right)} \leqslant K$.

4) При выполнении условий (15), (16) вместе с (35) (т.е. когда $\left\{\rho_{S}, R_{S}\right\}$ статически устойчиво) при достаточно малом $\varepsilon_{0}>0$ не существует другого решения $\left\{\widehat{\rho}_{S}, \widehat{R}_{S}\right\}$ такого, что $\left|R_{S}-\widehat{R}_{S}\right|<\varepsilon_{0}$. Это другое решение не предполагается положительньм.

ДоказАТЕЛьСтво. 1) В соответствии с замечанием $2 \rho_{S} \in C^{1}\left(\bar{\Omega}_{S}\right)$. Чтобы доказать (43), в силу неравенства (18) сначала получим

$$
p\left(\rho_{S}\right) \leqslant p_{\Gamma, S}+M r_{0}^{-k}\left\|f_{S}\right\|_{C\left(\bar{\Pi}_{S}\right)}, \quad \text { где } \Pi_{S}:=\left(r_{0}, \bar{R}_{S}\left(\delta_{S}\right)\right) \times J
$$

и поэтому $\rho_{S} \leqslant K_{0}($ так как $p(+\infty)=+\infty)$. Затем в силу $(23)$

$$
\left\|\left(\rho_{S}\right)_{r}\right\|_{C\left(\bar{\Omega}_{S}\right)} \leqslant \frac{1}{\min _{\left[\delta_{S}, K_{0}\right]} p^{\prime}(s)} K_{0}\left\|f_{S}\right\|_{C\left(\bar{\Pi}_{S}\right)} \leqslant K
$$

2 ) Вернемся к задаче Коши (20), (21). Если она имеет решение такое, что $p_{S}>0$ на $\bar{\Omega}_{S}$, то в силу условий (10) и (15) любое другое решение $\left(\widehat{p}_{S}, \widehat{m}_{S}\right)$ совпадает с $\left(p_{S}, m_{S}\right)$ на всяком сегменте $\left[R_{S}-\delta, R_{S}\right] \subset \bar{\Omega}_{S}$, где $\widehat{p}_{S}>0$, а поэтому и на всем $\bar{\Omega}_{S}$. Это обеспечивает отсутствие другого решения $\left\{\widehat{\rho}_{S}, \widehat{R}_{S}\right\}$ такого, что $\widehat{R}_{S}=R_{S}$.

Рассмотрев снова ту же задачу Коши, но теперь при $r>R_{S}$, можно продолжить $\rho_{S}$ и $m_{S}$ как функции из $C^{1}\left[r_{0}, \widetilde{R}_{S}\right]$ для $\widetilde{R}_{S}=R_{S}+\varepsilon_{S} \mathrm{c} \mathrm{min}{ }_{\left[r_{0}, \widetilde{R}_{S}\right]} \rho_{S} \geqslant \delta_{S} / 2$ и $\max _{\left[r_{0}, \widetilde{R}_{S}\right]} m_{S} \leqslant M_{1}-\varepsilon$ при достаточно малых $\varepsilon_{S}>0$ и $\varepsilon>0$; более того, это продолжение единственно при условии (15). Как следствие соотношения (7) удовлетворяются на $\left[r_{0}, \widetilde{R}_{S}\right]$. Рассуждения п. 1$)$ доказательства показьвают, что оценка $\left\|\rho_{S}\right\|_{C^{1}\left[r_{0}, \widetilde{R}_{S}\right]} \leqslant K_{1}$ также справедлива.

3) При выполнении условий (17) можно продифференцировать $(23)$ на $\left(r_{0}, \widetilde{R}_{S}\right)$ :

$$
\begin{aligned}
\left(\rho_{S}\right)_{r r}= & -\frac{p^{\prime \prime}\left(\rho_{S}\right)}{\left(p^{\prime}\left(\rho_{S}\right)\right)^{2}}\left(\rho_{S}\right)_{r} \rho_{S} f_{S}\left[m_{S}\right]+\frac{1}{p^{\prime}\left(\rho_{S}\right)}\left(\left(\rho_{S}\right)_{r} f_{S}\left[m_{S}\right]\right. \\
& \left.+\rho_{S}\left(f_{S}\right)_{r}\left[m_{S}\right]+\rho_{S}^{2} \varkappa\left(f_{S}\right)_{\chi}\left[m_{S}\right]\right),
\end{aligned}
$$

что и влечет п. 3). 
4) Чтобы доказать п. 4), будем рассуждать от противного. Предположим, что сушествует последовательность решений $\left\{\rho_{S, n}, R_{S, n}\right\}, n \geqslant 1$, такая, что $R_{S, n} \neq R_{S}$ (см. п. 2)) и $R_{S, n} \rightarrow R_{S}$ при $n \rightarrow \infty$. Тем самым получим последовательность

$$
\left(p_{S, n}, m_{S, n}\right)=\left(p\left(\rho_{S, n}\right), \int_{r_{0}}^{r}\left(\rho_{S, n} \varkappa\right)(q) d q\right)
$$

решений задачи Коши $(20),(21)$ с $R_{S, n}$ в роли $R_{S}$. Эти решения можно продолжить с $\Omega_{S, n}:=\left(r_{0}, R_{S, n}\right)$, удовлетворив (20) на большем интервале $\left(r_{0}, R_{S, n}+\varepsilon\right)$, с $\varepsilon \in\left(0, \varepsilon_{S}\right)$, не зависящим от $n$. В силу свойств последовательностей решений задачи Коши (см. опять [31; теорема I.2.4]) и единственности решений $\left(p_{S}, m_{S}\right)$ на $\left[r_{0}, R_{S}+\varepsilon_{S}\right]$ получим, что $\left\|p_{S, n}-p_{S}\right\|_{C\left[r_{0}, R_{S}+\varepsilon / 2\right]} \rightarrow 0$. Следовательно,

$$
\left\|\rho_{S, n}-\rho_{S}\right\|_{C\left[r_{0}, R_{S}+\varepsilon / 2\right]} \rightarrow 0
$$

и $\min _{\left[r_{0}, R_{S}+\varepsilon / 2\right]} \rho_{S, n} \geqslant \delta_{S} / 4$ при достаточно больших $n$.

По аналогии с (27) справедливо равенство

$$
\mathscr{B}_{R_{S, n}}\left[\rho_{S, n}, \rho_{S}\right]=0
$$

для формы

$$
\begin{aligned}
\mathscr{B}_{R_{S, n}}\left[\rho_{S, n}, \rho_{S}\right]= & \int_{\Omega_{S, n}}\left(\pi\left(\rho_{S, n}\right)-\pi\left(\rho_{S}\right)\right)\left(\rho_{S, n}-\rho_{S}\right) \varkappa d r \\
& -\left[\pi\left(\rho_{\Gamma, S}\right)-\pi\left(\rho_{S}\left(R_{S, n}\right)\right)\right]\left(m_{S, n}-m_{S}\right)\left(R_{S, n}\right) \\
& +\int_{\Omega_{S, n}}\left(f_{S}\left[m_{S, n}\right]-f_{S}\left[m_{S}\right]\right)\left(m_{S, n}-m_{S}\right) d r \\
=: & S_{1, n}+S_{2, n}+S_{3, n} .
\end{aligned}
$$

Проанализируем слагаемые $S_{1, n}, S_{2, n}$ и $S_{3, n}$. Во-первых, в силу равномерных двусторонних оценок для $\rho_{S, n}$ и $\rho_{S}$ на $\bar{\Omega}_{S, n}$ вместе с (10) и (44) имеем

$$
\begin{aligned}
S_{1, n} & =\int_{\Omega_{S, n}}\left\{\frac{p^{\prime}\left(\rho_{S}\right)}{\rho_{S}}+\alpha\left[\left\|\rho_{S, n}-\rho_{S}\right\|_{C\left(\bar{\Omega}_{S, n}\right)}\right]\right\}\left(\rho_{S, n}-\rho_{S}\right)^{2} \varkappa d r \\
& =\int_{\Omega_{S, n}} \frac{p^{\prime}\left(\rho_{S}\right)}{\rho_{S}}\left(\rho_{S, n}-\rho_{S}\right)^{2} \varkappa d r+o\left(\left\|\rho_{S, n}-\rho_{S}\right\|_{\Omega_{S, n}}^{2}\right) .
\end{aligned}
$$

Здесь и ниже $\alpha[z]$ - различные функции в интегралах по некоторым интервалам $E$, зависяшие от параметра $z$ и обладаюшие свойством $\|\alpha[z]\|_{C(\bar{E})} \rightarrow 0$ при $z \rightarrow 0^{+}$.

Во-вторых, в силу уравнения (26) на $\Omega_{S, n}$ и непрерывности $f_{S}$ и $\rho_{S}$ получим

$$
\begin{aligned}
\pi\left(\rho_{\Gamma, S}\right) & -\pi\left(\rho_{S}\left(R_{S, n}\right)\right)=\int_{R_{S, n}}^{R_{S}} f_{S}\left[m_{S}\right] d r \\
= & \int_{R_{S, n}}^{R_{S}} \rho_{S} \varkappa\left(\frac{f_{S}}{\rho_{S} \varkappa}\left(R_{S}, M\right)+\alpha\left[\left|R_{S, n}-R_{S}\right|\right]\right) d r
\end{aligned}
$$


поскольку $\left|M-m_{S}(r)\right| \leqslant\left|\int_{R_{S, n}}^{R_{S}} \rho_{S} \varkappa d r\right| \leqslant K\left|R_{S, n}-R_{S}\right|$ при $r$ между $R_{S, n}$ и $R_{S}$.

Поскольку также

$$
\int_{R_{S, n}}^{R_{S}} \rho_{S} \varkappa d r=\left(m_{S, n}-m_{S}\right)\left(R_{S, n}\right)
$$

то получим (ср. с (29))

$$
S_{2, n}=-\frac{f_{S}}{\varkappa \rho_{S}}\left(R_{S}, M\right)\left(m_{S, n}-m_{S}\right)^{2}\left(R_{S, n}\right)+o\left(\left|R_{S, n}-R_{S}\right|^{2}\right) .
$$

В-третьих, аналогично (47) выведем

$$
\begin{aligned}
S_{3, n}= & \int_{\Omega_{S, n}}\left\{\left(f_{S}\right)_{\chi}\left[m_{S}\right]+O\left(\theta\left[\left\|m_{S, n}-m_{S}\right\|_{C\left(\bar{\Omega}_{S, n}\right)}\right]\right)\right\}\left(m_{S, n}-m_{S}\right)^{2} d r \\
= & \int_{\Omega_{S, n}}\left(f_{S}\right)_{\chi}\left[m_{S}\right]\left(m_{S, n}-m_{S}\right)^{2} d r \\
& \quad+O\left(\left\|\theta\left[\left\|m_{S, n}-m_{S}\right\|_{C\left(\bar{\Omega}_{S, n}\right)}\right)\right\|_{L^{1}\left(\Omega_{S, n}\right)}\left\|m_{S, n}-m_{S}\right\|_{C\left(\bar{\Omega}_{S, n}\right)}^{2}\right) .
\end{aligned}
$$

Формула (48) влечет неравенства

$$
\begin{aligned}
\frac{\delta_{S}}{2} r_{0}^{k}\left|R_{S, n}-R_{S}\right| & \leqslant\left\|m_{S, n}-m_{S}\right\|_{C\left(\bar{\Omega}_{S, n}\right)} \\
& \leqslant\left\|\left(\rho_{S, n}-\rho_{S}\right) \varkappa\right\|_{L^{1}\left(\Omega_{S, n}\right)} \leqslant K\left\|\rho_{S, n}-\rho_{S}\right\|_{\Omega_{S, n}} .
\end{aligned}
$$

Поэтому, подставляя $(47),(49)$ и (50) в $(46)$, а также используя $(16)$, находим

$$
\mathscr{B}_{R_{S, n}}\left[\rho_{S, n}, \rho_{S}\right]=\mathscr{J}_{R_{S, n}}\left[m_{S, n}-m_{S}\right]+o\left(\left\|\rho_{S, n}-\rho_{S}\right\|_{\Omega_{S, n}}^{2}\right) .
$$

В силу утверждения 3 и условия (35) $\lambda_{\min }\left\{\rho_{S}, R_{S}\right\}\left(R_{S, n}\right) \geqslant \lambda_{\min }\left\{\rho_{S}, R_{S}\right\} / 2>0$ при достаточно больших $n \geqslant N$. Поэтому для таких $n$ согласно замечанию 4

$$
\mathscr{J}_{R_{S, n}}\left[m_{S, n}-m_{S}\right] \geqslant K^{-1}\left\|\rho_{S, n}-\rho_{S}\right\|_{\Omega_{S, n}}^{2}
$$

(где $K$ не зависит от $n$ ). Теперь (45), (52) и (53) влекут равенство $\rho_{S, n}=\rho_{S}$ на $\bar{\Omega}_{S, n}$ при $n \geqslant N$, которое противоречит ограничению на массу (9).

СлЕДСТВИЕ 1. Пусть $p^{\prime}>0$ на $\mathbb{R}^{+}$и при некотором $\delta_{S}>0$ справедливы условия (15) и (16) $с \bar{R}_{S}\left(\delta_{S}\right)$ в роли $\widetilde{R}_{S}$. Тогда может существовать только конечное число статически устойчивых решений таких, что $\left(\rho_{S}\right)_{\min } \geqslant \delta_{S}$.

ДокАЗАТЕЛЬСТво. Достаточно применить п. 4) с учетом замечания 2 (от противного).

Рассуждения из доказательства п. 4) относительно $\mathscr{B}_{R_{S, n}}\left[\rho_{S, n}, \rho_{S}\right]$ можно было бы заменить более простыми, но они будут существенно использованы в доказательстве оценок скорости стабилизации в следуюшем параграфе.

В силу замечания 5 условие

$$
\alpha_{0}:=\left\{\int_{\Omega_{S}}\left(\left(f_{S}\right)_{\chi}\left[m_{S}\right]\right)_{-} d r+\frac{\left(f_{S}\left(R_{S}, M\right)\right)_{+}}{\rho_{\Gamma, S} \varkappa\left(R_{S}\right)}\right\} \int_{\Omega_{S}} \frac{\rho_{S}}{p^{\prime}\left(\rho_{S}\right)} \varkappa d r \leqslant 1
$$


вместе с любым из двух условий

$$
\operatorname{meas}\left\{r \in \Omega_{S}:\left(f_{S}\right)_{\chi}\left[m_{S}\right](r) \neq 0\right\}>0, \quad \frac{f_{S}\left(R_{S}, M\right)}{\rho_{\Gamma, S} \varkappa\left(R_{S}\right)} \int_{\Omega_{S}} \frac{\rho_{S}}{p^{\prime}\left(\rho_{S}\right)} \varkappa d r \neq 1
$$

в случае $\alpha_{0}=1$ достаточно для того, чтобы получить $\lambda_{\min }\left\{\rho_{S}, R_{S}\right\}>0$.

Сравним эти условия с условиями из утверждения 2, п. 2). Во-первых, условие $(15)$ с $\bar{R}=\bar{R}_{S}\left(\delta_{S}\right)$ влечет, что $\psi \geqslant\left\|\left(\left(f_{S}\right)_{\chi}\right)_{-}\right\|_{C[0, M]}$ в $(24)$. Далее, при $f_{S} \leqslant 0$ получим

$$
\int_{\Omega_{S}} \frac{\rho_{S}}{p^{\prime}\left(\rho_{S}\right)} \varkappa d r \leqslant \frac{M}{C^{(1)}\left(\rho_{\Gamma, S}\right)}, \quad \alpha_{0} \leqslant \frac{M}{C^{(1)}\left(\rho_{\Gamma, S}\right)} \int_{r_{0}}^{\bar{R}_{S}\left(\rho_{\Gamma, S}\right)} \psi d r
$$

Таким образом, условие $(25)$ влечет $\alpha_{0} \leqslant 1$; более того, справедливо и второе условие (54).

Обратимся теперь к вариационной интерпретации стационарной задачи и условия статической устойчивости. Для положительных решений сначала исключим функцию $\rho_{S}$, используя равенство $\rho_{S}=\left(m_{S}\right)_{r} / \varkappa$, и перепишем эквивалентньм образом стационарную задачу как краевую задачу для ОДУ второго порядка в неизвестной области:

$$
\begin{gathered}
{\left[\pi\left(\frac{1}{\varkappa}\left(m_{S}\right)_{r}\right)\right]_{r}=f_{S}\left[m_{S}\right] \quad \text { в } \Omega_{S},} \\
m_{S}\left(r_{0}\right)=0,\left.\quad \pi\left(\frac{1}{\varkappa}\left(m_{S}\right)_{r}\right)\right|_{r=R_{S}}=\pi\left(\rho_{\Gamma, S}\right), \quad m_{S}\left(R_{S}\right)=M,
\end{gathered}
$$

с искомой функцией $m_{S} \in C^{1}\left(\bar{\Omega}_{S}\right)$ такой, что $\left(\left(m_{S}\right)_{r}\right)_{\min }>0$; фактически $m_{S} \in$ $C^{2}\left(\bar{\Omega}_{S}\right)$.

Чтобы упростить вычисления, выполним замену переменной

$$
\nu=\nu(r):=\frac{r^{k+1}}{k+1}
$$

и введем функции

$$
h(\nu(r), \chi):=\frac{f_{S}(r, \chi)}{r^{k}}, \quad H(\nu(r), \chi):=\int_{V_{0}}^{\nu(r)} h(\zeta, \chi) d \zeta=F(r, \chi), \quad V_{0}:=\frac{r_{0}^{k+1}}{k+1}
$$

Одновременно неравенства (12) и (22) принимают более простой вид

$$
\limsup _{\nu \rightarrow \infty} \frac{1}{\nu} \max _{0 \leqslant \chi \leqslant M} H(\nu, \chi) \leqslant f_{0}, \quad h(\nu, \chi) \leqslant f_{0}
$$

Таким образом, последняя задача преобразуется к более простой форме:

$$
\begin{gathered}
{\left[\pi\left(\widetilde{m}_{S}^{\prime}\right)\right]^{\prime}=h\left[\tilde{m}_{S}\right] \quad \text { в }\left(V_{0}, \nu_{S}\right),} \\
\tilde{m}_{S}\left(V_{0}\right)=0, \quad \pi\left(\widetilde{m}_{S}^{\prime}\left(\nu_{S}\right)\right)=\pi\left(\rho_{\Gamma, S}\right), \quad \tilde{m}_{S}\left(\nu_{S}\right)=M
\end{gathered}
$$


где $\widetilde{m}_{S}(\nu(r))=m_{S}(r), \varphi^{\prime}=d \varphi / d \nu$ и $\nu_{S}:=R_{S}^{k+1} /(k+1)$. Более того, статическая потенциальная энергия (введенная в [10]) преобразуется к виду

$$
\mathscr{F}_{S}\left\{\rho_{S}, R_{S}\right\}:=\int_{\Omega_{S}}\left(P_{1}\left(\rho_{S}\right)+p_{\Gamma, S}-\rho_{S} F\left[m_{S}\right]\right) \varkappa d r=\int_{V_{0}}^{\nu_{S}} g\left[\widetilde{m}_{S}, \widetilde{m}_{S}^{\prime}\right] d \nu
$$

где $g\left[\widetilde{m}_{S}, \widetilde{m}_{S}^{\prime}\right](\nu)=g\left(\nu, \widetilde{m}_{S}(\nu), \widetilde{m}_{S}^{\prime}(\nu)\right)$ с лагранжианом $g(\nu, m, n):=P_{1}(n)+$ $p_{\Gamma, S}-n H(\nu, m)$. Тем самьм получен классический функционал одномерного вариационного исчисления со свободным правым концомпри ограничении $\widetilde{m}_{S}\left(\nu_{S}\right)=M$.

Будем работать с этим функционалом следующим образом. При каком-либо $V_{1}>V_{0}$ введем подпространство $\widetilde{C}^{1}\left[V_{0}, V_{1}\right]:=\left\{w \in C^{1}\left[V_{0}, V_{1}\right]: w\left(V_{0}\right)=0\right\}$ и открытое множество $\mathscr{S}_{M}:=\left\{w \in \widetilde{C}^{1}\left[V_{0}, V_{1}\right]: \min _{\left[V_{0}, V_{1}\right]} w^{\prime}>0, w\left(V_{1}\right)>M\right\}$ и определим функционал

$$
\mathscr{R}[m]:=\int_{V_{0}}^{m^{-1}(M)} g\left[m, m^{\prime}\right] d \nu
$$

для $m \in \mathscr{S}_{M}$, где $m^{-1}$ - функция, обратная к $m$. Ясно, что $\mathscr{F}_{S}\left\{\rho_{S}, R_{S}\right\}=\mathscr{R}\left[\widetilde{m}_{S}\right]$.

Для $w \in \widetilde{C}^{1}\left[V_{0}, V_{1}\right]$ имеем $m+\tau w \in \mathscr{I}_{M}$ при $0 \leqslant \tau<\tau_{0}$ с достаточно малым $\tau_{0}=\tau_{0}(m, w)>0$. Заметим, что существует единственная функция $\widehat{\nu}$ такая, что $(m+\tau w)(\widehat{\nu}(\tau)) \equiv M$ на $\left[0, \tau_{0}\right) ;$ следовательно,

$$
\left.\left[\left(m^{\prime}+\tau w^{\prime}\right) \widehat{\nu}_{\tau}(\tau)+w\right]\right|_{\nu=\widehat{\nu}(\tau)} \equiv 0 \quad \text { на }\left[0, \tau_{0}\right),
$$

где $\widehat{\nu}_{\tau}=d \widehat{\nu} / d \tau$. Предположим, что выполнено условие $(15)$ с $r_{1}:=\left[(k+1) V_{1}\right]^{1 /(k+1)}$ в роли $\widetilde{R}_{S}$, и вычислим первую вариацию $\mathscr{R}$ :

$$
\begin{aligned}
\delta \mathscr{R}[m](w) & :=\left.\frac{d}{d \tau} \mathscr{R}[m+\tau w]\right|_{\tau=0} \\
& =\int_{V_{0}}^{\widehat{\nu}(0)}\left\{g_{n}\left[m, m^{\prime}\right] w^{\prime}+g_{m}\left[m, m^{\prime}\right] w\right\} d \nu+\left.g\left[m, m^{\prime}\right]\right|_{\nu=\widehat{\nu}(0)} \widehat{\nu}_{\tau}(0)
\end{aligned}
$$

где $g_{n}$ и $g_{m}$ - частные производные $g(\nu, m, n)$. Справедливы равенства

$$
\begin{aligned}
g_{n}(\nu, m, n) & =P_{0}(n)+\frac{p(n)}{n}-H(\nu, m)=\pi(n)+p(1)-H(\nu, m), \\
g_{m}(\nu, m, n) & =-n H_{\chi}(\nu, m) .
\end{aligned}
$$

Следовательно,

$$
g_{n}(\nu, m, n)-\frac{g(\nu, m, n)}{n}=\frac{p(n)-p_{\Gamma, S}}{n} .
$$

Ясно также, что $\widehat{\nu}_{0}:=\widehat{\nu}(0)=m^{-1}(M)$ и $\widehat{\nu}_{\tau}(0)=-\left(w / m^{\prime}\right)\left(\widehat{\nu}_{0}\right)$ (см. (59)). Поэтому

$$
\begin{aligned}
\delta \mathscr{R}[m](w)= & \int_{V_{0}}^{m^{-1}(M)}\left\{\left(\pi\left(m^{\prime}\right)-H[m]-\left.\left(\pi\left(m^{\prime}\right)-H[m]\right)\right|_{\nu=m^{-1}(M)}\right) w^{\prime}\right. \\
& \left.-m^{\prime} H_{\chi}[m] w\right\} d \nu+\left.\left\{\frac{p\left(m^{\prime}\right)-p_{\Gamma, S}}{m^{\prime}} w\right\}\right|_{\nu=m^{-1}(M)} .
\end{aligned}
$$


Задача о стационарных точках функционала $\mathscr{R}$, т.е.

$$
\delta \mathscr{R}\left[\widetilde{m}_{S}\right](w)=0 \quad \forall w \in \widetilde{C}^{1}\left[V_{0}, V_{1}\right]
$$

эквивалентна уравнению Эйлера (56) для $\mathscr{R}$ в совокупности с соотношениями (57) (принимая во внимание равенство $\left.(H[m])^{\prime}-m^{\prime} H_{\chi}[m]=h[m]\right)$. Таким образом, решения $\widetilde{m}_{S}$ стационарной задачи $(56),(57)$, и только они, являются стационарными точками $\mathscr{R}$.

Далее, предположим также, что $H_{\chi \chi} \in L^{1}\left(V_{0}, V_{1} ; C\left[0, M_{1}\right]\right.$ ) (при некотором $\left.M_{1}>M\right)$ и $m, w \in C^{2}\left[V_{0}, V_{1}\right]$, и выгислим вторую вариацию $\mathscr{R}:$

$$
\begin{aligned}
\delta^{2} \mathscr{R}[m](w):=\left.\frac{d^{2}}{d \tau^{2}} \mathscr{R}[m+\tau w]\right|_{\tau=0} \\
=\int_{V_{0}}^{\widehat{\nu}_{0}}\left\{g_{n n}\left[m, m^{\prime}\right]\left(w^{\prime}\right)^{2}+2 g_{m n}\left[m, m^{\prime}\right] w w^{\prime}+g_{m m}\left[m, m^{\prime}\right] w^{2}\right\} d \nu \\
\quad+\left\{2\left(g_{n}\left[m, m^{\prime}\right] w^{\prime}+g_{m}\left[m, m^{\prime}\right] w\right) \widehat{\nu}_{\tau}(0)\right. \\
\left.\quad+\left(g_{\nu}\left[m, m^{\prime}\right]+g_{m}\left[m, m^{\prime}\right] m^{\prime}+g_{n}\left[m, m^{\prime}\right] m^{\prime \prime}\right) \widehat{\nu}_{\tau}^{2}(0)+g\left[m, m^{\prime}\right] \widehat{\nu}_{\tau \tau}(0)\right\}\left.\right|_{\nu=\widehat{\nu}_{0}}
\end{aligned}
$$

Продифференцировав (59), получим

$$
\widehat{\nu}_{\tau \tau}(0)=\left.\left\{-\frac{m^{\prime \prime}}{m^{\prime}} \widehat{\nu}_{\tau}^{2}(0)-2 \frac{w^{\prime}}{m^{\prime}} \widehat{\nu}_{\tau}(0)\right\}\right|_{\nu=\widehat{\nu}_{0}} .
$$

Поэтому

$$
\begin{aligned}
\delta^{2} \mathscr{R}[m](w)= & \int_{V_{0}}^{\widehat{\nu}_{0}}\left\{g_{n n}\left[m, m^{\prime}\right]\left(w^{\prime}\right)^{2}+\left(g_{m m}\left[m, m^{\prime}\right]-\left(g_{m n}\left[m, m^{\prime}\right]\right)^{\prime}\right) w^{2}\right\} d \nu \\
& +\left\{\left(g_{n}\left[m, m^{\prime}\right]-\frac{g\left[m, m^{\prime}\right]}{m^{\prime}}\right)\left(2 w^{\prime}-\frac{m^{\prime \prime} w}{m^{\prime}}\right)\left(-\frac{w}{m^{\prime}}\right)\right. \\
& \left.+\left(g_{m n}\left[m, m^{\prime}\right]-\frac{g_{m}\left[m, m^{\prime}\right]}{m^{\prime}}+\frac{g_{\nu}\left[m, m^{\prime}\right]}{\left(m^{\prime}\right)^{2}}\right) w^{2}\right\}\left.\right|_{\nu=\widehat{\nu}_{0}} .
\end{aligned}
$$

Имеем

$$
\begin{gathered}
g_{n n}(\nu, m, n)=\frac{p^{\prime}(n)}{n}, \quad g_{m n}(\nu, m, n)=\frac{g_{m}(\nu, m, n)}{n}, \\
g_{\nu}(\nu, m, n)=n h(\nu, m), \quad g_{m m}\left[m, m^{\prime}\right]-\left(g_{m n}\left[m, m^{\prime}\right]\right)^{\prime}=-h_{\chi}[m] .
\end{gathered}
$$

Следовательно, в случае, когда $m^{\prime}\left(m^{-1}(M)\right)=\rho_{\Gamma, S}$, выводим

$$
\delta^{2} \mathscr{R}[m](w)=\int_{V_{0}}^{m^{-1}(M)}\left\{\frac{p^{\prime}\left(m^{\prime}\right)}{m^{\prime}}\left(w^{\prime}\right)^{2}-h_{\chi}[m] w^{2}\right\} d \nu-\left.\left\{\frac{h[m]}{m^{\prime}} w^{2}\right\}\right|_{\nu=m^{-1}(M)}
$$

Окончательно

$$
\delta^{2} \mathscr{R}\left[\widetilde{m}_{S}\right](\widetilde{w})=\mathscr{J}_{R_{S}}\left\{\rho_{S}, R_{S}\right\}[w]
$$

с $\widetilde{w}(\nu(r))=w(r)$, и, таким образом, условие статической устойчивости (35) означает положительность второй вариации

$$
\delta^{2} \mathscr{R}\left[\widetilde{m}_{S}\right](w)>0 \quad \forall w \in C^{2}\left[V_{0}, V_{1}\right], \quad w\left(V_{0}\right)=0, \quad w \not \equiv 0,
$$

при $p^{\prime}>0, p^{\prime \prime} \in C^{2}\left(\mathbb{R}^{+}\right)$и $\left(f_{S}\right)_{\chi} \in C\left(\bar{\Omega}_{S} \times[0, M]\right)$ (напомним предположение, что $V_{1}$ удовлетворяет условию $\left.\widetilde{m}_{S}\left(V_{1}\right)>M\right)$.

Отметим, что к ограничению на массу для функционала (58) можно было также применить метод множителей Лагранжа. 


\section{§3. Оценки скорости стабилизации в эйлеровых координатах}

Обратимся к нестационарной задаче (1)-(6) и дополнительно к условиям (10) на $p$ и (11) на $f_{S}$ предположим, что коэффициенты вязкости $\mu, \mu_{1} \in C\left(\mathbb{R}^{+}\right)$имеют $\mu^{\prime}, \mu_{1}^{\prime} \in L_{\mathrm{loc}}^{\infty}\left(\mathbb{R}^{+}\right)$и удовлетворяют неравенствам

$$
0<\underline{\mu} \leqslant \mu(s), \quad-\underline{\mu}_{1} \leqslant \mu_{1}(s) \leqslant \bar{\mu}_{1} \text { при } s>0, \quad 2 k \bar{\mu}_{1}<(k+1) \underline{\mu},
$$

где $\underline{\mu}_{1} \geqslant 0, \bar{\mu}_{1} \geqslant 0$. Напомним, что последнее условие (61), связывающее $\mu$ и $\mu_{1}$, является стандартным при постоянных $\mu$ и $\mu_{1}$. Предположим также, что $\Delta f \in$ $L_{\text {loc }}^{1}\left(\mathbb{R}^{+} ; C_{b}\left(\bar{\Pi}_{\infty}\right)\right)$ удовлетворяет условиям

$$
|\Delta f(r, \chi, t)| \leqslant \bar{f}_{1}(t)+\bar{f}_{2}(t) \quad \text { c }\left\|\bar{f}_{1}\right\|_{L^{1}\left(\mathbb{R}^{+}\right)}+\left\|\bar{f}_{2}\right\|_{L^{2}\left(\mathbb{R}^{+}\right)} \leqslant N, \bar{f}_{1} \geqslant 0, \bar{f}_{2} \geqslant 0 .
$$

Здесь и ниже $C_{b}(\bar{G})$ для неограниченной области $G$ обозначает пространство функций, непрерьвных и ограниченных на $\bar{G}$, с нормой $\|\varphi\|_{C_{b}(\bar{G})}:=\sup _{\bar{G}}|\varphi|$.

Будем изучать стабилизацию сильного решения задачи со свободной границей (1)-(6), обладающего свойствами

$$
\begin{array}{lrl}
\rho \in C\left(\bar{Q}_{T}\right), & \rho_{r}, \rho_{t} \in L^{2, \infty}\left(Q_{T}\right), & \min _{\bar{Q}_{T}} \rho>0, \\
v \in H^{2,1}\left(Q_{T}\right), & R \in C^{1}[0, T], & \min _{[0, T]} R>r_{0}
\end{array}
$$

при всех $T>0$, где $Q_{T}:=\{(r, t) \in Q: t<T\}$. Как следствие $v \in C\left(\bar{Q}_{T}\right)$ и $m \in C^{1}\left(\bar{Q}_{T}\right)$.

Здесь и ниже $L^{\lambda, \nu}\left(Q_{T}\right)$ и $H^{2,1}\left(Q_{T}\right)=W_{2}^{2,1}\left(Q_{T}\right)$ - классические анизотропные пространства Лебега и Соболева [34] с нормами

$$
\|\varphi\|_{L^{\lambda, \nu}\left(Q_{T}\right)}=\|\| \varphi\left\|_{L^{\lambda}\left(\Omega_{t}\right)}\right\|_{L^{\nu}(0, T)}
$$

где $\Omega_{t}:=\left(r_{0}, R(t)\right)$ и $\lambda, \nu \in[1, \infty]$, и

$$
\|\varphi\|_{H^{2,1}\left(Q_{T}\right)}=\|\varphi\|_{V_{2}\left(Q_{T}\right)}+\left\|\varphi_{r}\right\|_{V_{2}\left(Q_{T}\right)}+\left\|\varphi_{t}\right\|_{L^{2}\left(Q_{T}\right)}
$$

где $V_{2}\left(Q_{T}\right)$ - стандартное пространство функций $\varphi$, имеющих конечную (параболическую) энергию $\|\varphi\|_{V_{2}\left(Q_{T}\right)}=\|\varphi\|_{L^{2, \infty}\left(Q_{T}\right)}+\left\|\varphi_{r}\right\|_{L^{2}\left(Q_{T}\right)}$. Будем использовать те же пространства и с $Q$ в роли $Q_{T}$.

Предположим, что $\rho^{0}, v^{0} \in H^{1}\left(\Omega_{0}\right), \min _{\bar{\Omega}_{0}} \rho^{0}>0, v^{0}\left(r_{0}\right)=0$ и $\Delta p_{\Gamma} \in L^{2}\left(\mathbb{R}^{+}\right) ;$ эти условия необходимы для существования сильного решения.

Напомним хорошо известные законы сохранения массы и энергии

$$
\begin{gathered}
\frac{d}{d t} \int_{\Omega_{t}} \rho \varkappa d r=0 \quad\left(\text { т.е. } \int_{\Omega_{t}} \rho \varkappa d r \equiv M\right) \\
\frac{d}{d t}(\mathscr{E}+\mathscr{F}[\rho])+\int_{\Omega_{t}} \frac{\mu(\rho)}{\varkappa}(\varkappa v)_{r}^{2} d r-2 k\left(\mu_{1}(\rho) \frac{\varkappa v^{2}}{r}\right)_{R} \\
=\int_{\Omega_{t}} \rho \Delta f[m] v \varkappa d r-\Delta p_{\Gamma}(v \varkappa)_{R},
\end{gathered}
$$


вьполненные (почти всюду) на $\mathbb{R}^{+}$, где

$$
\mathscr{E}:=\frac{1}{2} \int_{\Omega_{t}} \rho v^{2} \varkappa d r \quad \text { и } \mathscr{F}[\rho]:=\int_{\Omega_{t}}\left(\rho P_{0}(\rho)+p_{\Gamma, S}-\rho F[m]\right) \varkappa d r
$$

являются кинетической и потенциальной энергиями (последняя - с точностью до аддитивной постоянной и в пренебрежении возмущениями $\Delta f$ и $\left.\Delta p_{\Gamma}\right)$, причем приняты обозначения $F[m](r, t):=F(r, m(r, t)), \psi_{r}^{2}:=\left(\psi_{r}\right)^{2}$ и $\varphi_{R}:=\left.\varphi\right|_{r=R(t)}$. Вывод второго закона использует формулу

$$
\frac{d}{d t} \int_{\Omega_{t}} \rho \varphi \varkappa d r=\int_{\Omega_{t}} \rho\left(D_{t} \varphi\right) \varkappa d r \quad \forall \varphi \in W^{1,1}\left(Q_{T}\right) \quad \text { и при почти всех } t \in(0, T),
$$

содержашую лагранжеву производную по времени $D_{t} \varphi:=\varphi_{t}+v \varphi_{r}$; эта формула будет применяться и в дальнейшем. Здесь и ниже аргументы $(r, t)$ функций в интегралах и нормах по $\Omega_{t}$ опускаются.

Теперь извлечем некоторые результаты из [10], используемые в дальнейшем, причем слегка модифицируем их. Положим $\widetilde{\rho}:=\rho$ в $\bar{Q}$ и $\widetilde{\rho}(r, t):=\rho(R(t), t)$ при $r>R(t)$ и $t \geqslant 0$.

Теорема 1. 1) Пусть выполнены условия на $p, \mu, \mu_{1} u f_{S}, \Delta f$, перечисленнъе в начале параграфа, вместе с условиями

$$
0<\rho^{0} \leqslant N, \quad\left\|v^{0}\right\|_{L^{2}\left(\Omega_{0}\right)} \leqslant N
$$

(12), (13) и $\left\|\Delta p_{\Gamma}\right\|_{L^{2}\left(\mathbb{R}^{+}\right)} \leqslant N$. Тогда верны следующие оченки, включая равномерную оценку сверху для $\rho$ и двусторонние оценки для $R$ :

$$
\begin{gathered}
\|\sqrt{\rho} v\|_{L^{2, \infty}(Q)}+\left\|(\varkappa v)_{r}\right\|_{L^{2}(Q)} \leqslant K, \quad \rho(r, t) \leqslant \bar{\rho}:=K \quad \text { в } \bar{Q}, \\
r_{0}+K^{-1} \leqslant R(t) \leqslant \bar{R}:=K \quad \text { на } \overline{\mathbb{R}}^{+} .
\end{gathered}
$$

Kроме того, $\mathscr{E}(t) \rightarrow 0$ u $\mathscr{F}[\rho](t) \rightarrow \mathscr{F}(S)$ nрu $t \rightarrow \infty$.

2) Пусть дополнительно выполнены условие (14) вместе с условиями

$$
s p^{\prime}(s)=O(1) \quad u \quad \mu(s)=O(1) \quad \text { npu } \quad s \rightarrow 0^{+} .
$$

Тогда для любой последовательности $t_{n} \rightarrow+\infty$ существует подпоследовательность $\theta_{n}$ такая, что

$$
\widetilde{\rho}\left(\cdot, \theta_{n}\right) \rightarrow \rho_{*}(\cdot) \quad \text { *-слабо в } \quad L^{\infty}\left(r_{0}, \bar{R}\right) \quad \text { u } \quad R\left(\theta_{n}\right) \rightarrow R_{*} .
$$

Более того, для любой последовательности $\theta_{n} \rightarrow \infty, \theta_{n} \geqslant 0$, удовлетворяющей этим свойствам, пара $\left\{\left.\rho_{*}\right|_{\left(r_{0}, R_{*}\right)}, R_{*}\right\}=\left\{\rho_{S}, R_{S}\right\}$ служстт стационарным решением, удовлетворяющим равенству

$$
\mathscr{F}_{S}\left\{\rho_{S}, R_{S}\right\}=\mathscr{F}^{(S)},
$$

и справедливы предельные соотношения

$$
\widetilde{\rho}\left(\cdot, \theta_{n}\right) \rightarrow \rho_{S}(\cdot) \quad \text { в } \quad L^{\lambda}\left(\Omega_{S}\right) \quad \forall 1 \leqslant \lambda<\infty \quad u \quad R\left(\theta_{n}\right) \rightarrow R_{S}
$$


3) Пусть выполнены условия пп. 1) и 2) и $p^{\prime}>0$ на $\mathbb{R}^{+}$. Предположим, что среди стационарных решений, удовлетворяющих (67) и (68) с $\lambda=1$ для некоторой последовательности $\theta_{n} \rightarrow \infty, \theta_{n} \geqslant 0$, существует положительное (такое, что $\left(\rho_{S}\right)_{\min } \geqslant \delta_{S}$ при некотором $\left.\delta_{S}>0\right)$ и статически устойчивое решение и выполнены также условия $(15),(16)$ на $f_{S}$. Тогда это решение является единственным, удовлетворяющим (68), и поэтому

$$
\widetilde{\rho}(\cdot, t) \rightarrow \rho_{S}(\cdot) \quad \text { в } L^{\lambda}\left(\Omega_{S}\right) \quad \forall 1 \leqslant \lambda<\infty \quad \text { nри } t \rightarrow \infty .
$$

Кроме того, если $N^{-1} \leqslant \rho^{0}$, то справедлива равномерная оценка снизу для $\rho$

$$
0<\underline{\rho} \leqslant \rho(r, t) \quad \text { в } \quad \bar{Q}
$$

и оченка

$$
\|v\|_{V_{2}(Q)} \leqslant K .
$$

4) Пусть выполнены все условия пп. 1)-3) вместе $c$

$$
\left\|\rho_{r}^{0}\right\|_{L^{2}\left(\Omega_{0}\right)} \leqslant N
$$

Тогда справедливы равномерная $H^{1}$-оценка и $C$-стабилизация для $\rho$ :

$$
\begin{gathered}
\sup _{t \geqslant 0}\|\rho(\cdot, t)\|_{H^{1}\left(\Omega_{t}\right)} \leqslant K, \\
\left\|\rho(\cdot, t)-\rho_{S}(\cdot)\right\|_{C\left(\bar{\Omega}_{t}\right)} \rightarrow 0 \quad \text { npu } \quad t \rightarrow \infty .
\end{gathered}
$$

5) Пусть выполнены все условия пп. 1)-4) вместе с

$$
\left\|v_{r}^{0}\right\|_{L^{2}\left(\Omega_{0}\right)} \leqslant N, \quad \bar{f}_{1}=0 \quad(c \mathcal{M} .(62)), \quad\left\|p_{\Gamma}^{\prime}\right\|_{L^{2}\left(\mathbb{R}^{+}\right)} \leqslant N .
$$

Тогда справедливы равномерная $H^{1}$-оценка для $v$ и $L^{2}$-оченка для $\rho_{t}$ :

$$
\begin{gathered}
\|v\|_{C_{b}(\bar{Q})} \leqslant K_{1} \sup _{t \geqslant 0}\|v(\cdot, t)\|_{H^{1}\left(\Omega_{t}\right)} \leqslant K, \\
\quad \operatorname{ess} \sup _{t>0}\left\|\rho_{t}(\cdot, t)\right\|_{L^{2}\left(\Omega_{t}\right)} \leqslant K .
\end{gathered}
$$

В оченках (71) и ниже в 33 величинь $K$ и $K_{i}$ зависят и от $\underline{\rho}$.

Справедливость этих результатов следует из [10; теоремы 1,3 и 5], см. также [10; замечания 2 и 3 , следствие 3]. Для вывода п. 3 ) используется также последний пункт утверждения 4 настоящей статьи, гарантирующий, что $\omega$-предельное множество для пары $\{\rho, R\}$ сводится к единственной точке $\left\{\rho_{S}, R_{S}\right\}$. Кроме того, в этой ситуации оценка (70) справедлива при $\left(\rho_{S}\right)_{\min } \geqslant \delta_{S}$ без аналогичного ограничения на любое стационарное решение, как в [10; теорема 5, п. 1].

Отметим, что в простейшем случае, когда стационарное решение единственно, свойство (69) заведомо вьполняется.

Теперь можно сформулировать и доказать основной результат параграфа. 
ТЕОРЕМА 2. 1) Пусть выполнены все условия пп. 1)-4) теоремы 1 вместе с условием на $\left(f_{S}\right)_{\chi}$ :

$\left(f_{S}\right)_{\chi}$ непрерьвна в некоторой окрестности точки $\left(R_{S}, M\right)$.

Тогда верны следующие утверждения:

а) справедлива $L^{2}\left(\Omega_{t}\right)$-оценка скорости стабилизации

$$
\begin{aligned}
\delta_{0}(t) & :=\left\|\rho(\cdot, t)-\rho_{S}(\cdot)\right\|_{L^{2}\left(\Omega_{t}\right)}+\|v(\cdot, t)\|_{L^{2}\left(\Omega_{t}\right)}+\left|\rho(R(t), t)-\rho_{\Gamma, S}\right| \\
& \leqslant K\left(e^{-a_{0}\left(t-t_{0}\right)} \delta_{0}\left(t_{0}\right)+\left\|e^{a_{0}(\tau-t)}\right\| \Delta f[m](\cdot, \tau)\left\|_{L^{2}\left(\Omega_{\tau}\right)}\right\|_{L^{1}\left(t_{0}, t\right)}\right. \\
& \left.+\left\|e^{a_{0}(\tau-t)} \Delta p_{\Gamma}(\tau)\right\|_{L^{2}\left(t_{0}, t\right)}\right) \quad \text { npu } \quad t \geqslant t_{0}
\end{aligned}
$$

б) при выполнении дополнительных условий (17) справедлива комбинированная оченка скорости стабилизачии

$$
\begin{gathered}
\delta_{1}(t):=\left\|\rho(\cdot, t)-\rho_{S}(\cdot)\right\|_{H^{1}\left(\Omega_{t}\right)}+\|v(\cdot, t)\|_{L^{2}\left(\Omega_{t}\right)}+\left|\rho(R(t), t)-\rho_{\Gamma, S}\right| \\
\leqslant K\left(e^{-a_{1}\left(t-t_{0}\right)} \delta_{1}\left(t_{0}\right)+\left\|e^{a_{1}(\tau-t)}\right\| \Delta f[m](\cdot, \tau)\left\|_{L^{2}\left(\Omega_{\tau}\right)}\right\|_{L^{1}\left(t_{0}, t\right)}\right. \\
\left.\quad+\left\|e^{a_{1}(\tau-t)} \Delta p_{\Gamma}(\tau)\right\|_{L^{2}\left(t_{0}, t\right)}\right) \quad \text { npu } \quad t \geqslant t_{0}
\end{gathered}
$$

в) при выполнении дополнительных условий (17) и (75) справедлива $H^{1}\left(\Omega_{t}\right)$-оценка скорости стабилизации

$$
\begin{aligned}
\delta_{2}(t):= & \left\|\rho(\cdot, t)-\rho_{S}(\cdot)\right\|_{H^{1}\left(\Omega_{t}\right)}+\|v(\cdot, t)\|_{H^{1}\left(\Omega_{t}\right)}+\left|\rho(R(t), t)-\rho_{\Gamma, S}\right| \\
\leqslant & K\left(e^{-a_{2}\left(t-t_{0}\right)}\left(\delta_{2}\left(t_{0}\right)+\left|\Delta p_{\Gamma}\left(t_{0}\right)\right|\right)+\left\|e^{a_{2}(\tau-t)}\right\| \Delta f[m](\cdot, \tau)\left\|_{L^{2}\left(\Omega_{\tau}\right)}\right\|_{L^{2}\left(t_{0}, t\right)}\right. \\
& \left.+\left\|e^{a_{2}(\tau-t)} \Delta p_{\Gamma}(\tau)\right\|_{L^{2}\left(t_{0}, t\right)}+\left\|e^{a_{2}(\tau-t)} p_{\Gamma}^{\prime}(\tau)\right\|_{L^{1}\left(t_{0}, t\right)}\right) \quad n p u \quad t \geqslant t_{0} . \quad(81)
\end{aligned}
$$

B nn. а)-в) $a_{i}:=1 / K_{i}, i=0,1,2$, a $t_{0} \geqslant 0$ достаточно велико.

2) Пусть $\left\{\rho_{S}, R_{S}\right\}$ - любое статически устойчивое положительное стачионарное решение (в отличие от условий на него в теореме $1, n .3)$ ). Если данные удовлетворяют следующему условию близости к стационарным:

$$
\begin{aligned}
& \left\|\rho^{0}-\rho_{S}\right\|_{L^{2}\left(\Omega_{0}\right)}+\left|R_{0}-R_{S}\right|+\left\|v^{0}\right\|_{L^{2}\left(\Omega_{0}\right)} \\
& \quad+\left\|\bar{f}_{1}\right\|_{L^{1}\left(\mathbb{R}^{+}\right)}+\left\|\bar{f}_{2}\right\|_{L^{2}\left(\mathbb{R}^{+}\right)}+\left\|\Delta p_{\Gamma}\right\|_{L^{2}\left(\mathbb{R}^{+}\right)} \leqslant \varepsilon_{0}
\end{aligned}
$$

с достаточно мальм $\varepsilon_{0}>0$, то п. 1) справедлив при $t_{0}=0$, т.е. при любих $t \geqslant 0$, причем величины $K$ и $a_{0}, a_{1}, a_{2}$ не зависят от данных; в этом смысле $\left\{\rho_{S}, R_{S}\right\}$ динамически устойчиво.

Этот результат верен и при немонотонной функиии состояния $p$ (опуская второе условие (10)) при условии, что значения $\rho_{S}$ на $\bar{\Omega}_{S}$ принадлежсат какому-либо интервалу $\left(s_{1}, s_{2}\right)$ устойчивости $p$, где $p^{\prime}>0$.

ЗАмЕчАниЕ 6 . В силу неравенства

$$
\left|R-R_{S}\right| \leqslant K\left\|\rho-\rho_{S}\right\|_{L^{2}\left(\Omega_{t}\right)} \text { при } t \geqslant t_{0}
$$

(см. его доказательство ниже) в левую часть оценки (79) можно добавить слагаемoe $\left|R(t)-R_{S}\right|$. 
ДокАЗАТЕЛЬСтво. Шаг 1. Сначала вернемся к закону сохранения энергии и преобразуем член $\frac{d}{d t} \mathscr{F}[\rho]$. При любых положительных $s$ и $s_{0}$ имеем

$$
\begin{gathered}
s P_{0}(s)=s \pi(s)-p(s)+s p(1)=\int_{s_{0}}^{s} \frac{s-\zeta}{\zeta} p^{\prime}(\zeta) d \zeta-p\left(s_{0}\right)+s\left(\pi\left(s_{0}\right)+p(1)\right) \\
=\left(s-s_{0}\right)^{2} \Pi_{1}\left(s, s_{0}\right)+s \pi\left(s_{0}\right)-p\left(s_{0}\right)+s p(1), \\
\quad \Pi_{1}\left(s, s_{0}\right):=\left.\int_{0}^{1} \frac{p^{\prime}(\zeta)}{\zeta}\right|_{\zeta=(1-\lambda) s_{0}+\lambda s}(1-\lambda) d \lambda .
\end{gathered}
$$

Следовательно,

$\rho P_{0}(\rho)+p_{\Gamma, S}-\rho F[m]=\left(\rho-\rho_{S}\right)^{2} \Pi_{1}\left(\rho, \rho_{S}\right)+\rho\left(\pi\left(\rho_{S}\right)-F[m]\right)+p_{\Gamma, S}-p\left(\rho_{S}\right)+\rho p(1)$.

Будем использовать свойства стационарного решения, описанные в утверждении 4 , п. 1), вместе со свойством равномерной стабилизации (74) и предположим, что $t$ настолько велико, что $R(t) \leqslant R_{S}+\varepsilon_{S}$. В силу (63) и стационарного уравнения (26) аналогично (50) получим

$$
\begin{aligned}
& \frac{d}{d t} \int_{\Omega_{t}} \rho\left(\pi\left(\rho_{S}\right)-F[m]\right) \varkappa d r=\int_{\Omega_{t}} \rho v\left(f_{S}\left[m_{S}\right]-f_{S}[m]\right) \varkappa d r \\
& \quad=-\int_{\Omega_{t}} \rho v\left\{\left(f_{S}\right)_{\chi}\left[m_{S}\right]\left(m-m_{S}\right)+O\left(\theta\left[\left\|m-m_{S}\right\|_{C\left(\bar{\Omega}_{t}\right)}\right]\right)\left\|m-m_{S}\right\|_{C\left(\bar{\Omega}_{t}\right)}\right\} \varkappa d r .
\end{aligned}
$$

Далее, применив равенство $\left(m-m_{S}\right)_{t}=-\rho v \varkappa$ и условия (78) и (16), выведем

$$
\begin{aligned}
\frac{d}{d t} \int_{\Omega_{t}} \rho( & \left.\pi\left(\rho_{S}\right)-F[m]\right) \varkappa d r \\
= & \frac{d}{d t} \frac{1}{2} \int_{\Omega_{t}}\left(f_{S}\right)_{\chi}\left[m_{S}\right]\left(m-m_{S}\right)^{2} d r-\frac{1}{2}\left\{v\left(f_{S}\right)_{\chi}\left[m_{S}\right]\left(m-m_{S}\right)^{2}\right\}_{R} \\
& +O\left(\left\|\theta\left[\left\|m-m_{S}\right\|_{C\left(\bar{\Omega}_{t}\right)}\right]\right\|_{L^{1}\left(\Omega_{t}\right)}\right) \bar{\rho}\|v\|_{C\left(\bar{\Omega}_{t}\right)}\left\|m-m_{S}\right\|_{C\left(\bar{\Omega}_{t}\right)} \\
= & \frac{d}{d t} \frac{1}{2} \int_{\Omega_{t}}\left(f_{S}\right)_{\chi}\left[m_{S}\right]\left(m-m_{S}\right)^{2} d r+\|v\|_{C\left(\bar{\Omega}_{t}\right)} o\left(\left\|m-m_{S}\right\|_{C\left(\bar{\Omega}_{t}\right)}\right) .
\end{aligned}
$$

Ясно также, что

$\frac{d}{d t} \int_{\Omega_{t}}\left(p_{\Gamma, S}-p\left(\rho_{S}\right)\right) \varkappa d r=\frac{d}{d t} \mathscr{A}_{S}, \quad \mathscr{A}_{S}:=\mathscr{A}_{S}\left(R, R_{S}\right)=\int_{R_{S}}^{R}\left(p_{\Gamma, S}-p\left(\rho_{S}\right)\right) \varkappa d r$.

В итоге, используя закон сохранения массы, имеем

$$
\frac{d}{d t} \mathscr{F}[\rho]=\frac{d}{d t}\left(\mathscr{U}+\mathscr{A}_{S}\right)+\|v\|_{C\left(\bar{\Omega}_{t}\right)} o\left(\left\|m-m_{S}\right\|_{C\left(\bar{\Omega}_{t}\right)}\right),
$$

где

$$
\mathscr{U}:=\int_{\Omega_{t}}\left(\Pi_{1}\left(\rho, \rho_{S}\right)\left(\rho-\rho_{S}\right)^{2} \varkappa+\frac{1}{2}\left(f_{S}\right)_{\chi}\left[m_{S}\right]\left(m-m_{S}\right)^{2}\right) d r .
$$


Теперь из закона сохранения энергии, применив неравенства

$$
\begin{gathered}
\varepsilon_{2} \underline{\mu}\left\|\frac{1}{\sqrt{\varkappa}}(\varkappa v)_{r}\right\|_{\Omega_{t}}^{2} \leqslant \int_{\Omega_{t}} \frac{\mu(\rho)}{\varkappa}(\varkappa v)_{r}^{2} d r-\left(\tilde{\mu}_{1}(\rho) \frac{\varkappa v^{2}}{r}\right)_{R}, \\
r_{0}^{k}\|v\|_{C\left(\bar{\Omega}_{t}\right)} \leqslant\left\|(\varkappa v)_{r}\right\|_{L^{1}\left(\Omega_{t}\right)} \leqslant K_{0}^{1 / 2}\left\|\frac{1}{\sqrt{\varkappa}}(\varkappa v)_{r}\right\|_{\Omega_{t}}
\end{gathered}
$$

с $\tilde{\mu}_{1}:=2 k \mu_{1}$ и достаточно мальм $\varepsilon_{2}>0$ (см. [10; доказательство теоремы 1, п. 1$\left.]\right)$, выведем

$$
\begin{aligned}
\frac{d}{d t}(\mathscr{E}+ & \left.\mathscr{U}+\mathscr{A}_{S}\right)+K^{-1}\left\|(\varkappa v)_{r}\right\|_{\Omega_{t}}^{2} \\
& \leqslant o\left(\left\|m-m_{S}\right\|_{C\left(\bar{\Omega}_{t}\right)}^{2}\right)+K\left(\|\Delta f[m]\|_{\Omega_{t}} \mathscr{E}^{1 / 2}+\left(\Delta p_{\Gamma}\right)^{2}\right)
\end{aligned}
$$

Будем использовать следующие соотношения (аналогичные (51) и (48)):

$$
\begin{gathered}
\bar{R}^{-1 / 2}\left\|m-m_{S}\right\|_{L^{2}\left(\Omega_{t}\right)} \leqslant\left\|m-m_{S}\right\|_{C\left(\bar{\Omega}_{t}\right)} \leqslant \int_{\Omega_{t}}\left|\rho-\rho_{S}\right| \varkappa d r \\
\leqslant \bar{R}^{k+1 / 2}\left\|\rho-\rho_{S}\right\|_{L^{2}\left(\Omega_{t}\right)} \\
\int_{R_{S}}^{R} \rho_{S} \varkappa d r=\int_{\Omega_{t}} \rho_{S} \varkappa d r-M=-\left(m-m_{S}\right)_{R}
\end{gathered}
$$

они влекут неравенство (83) (с $\left.K:=2 \bar{R}^{k+1 / 2} /\left(\delta_{S} r_{0}^{k}\right)\right)$. Заметим, что в силу условия (15) и неравенств (86) и (83) справедлива оценка сверху

$$
\begin{aligned}
\mathscr{E}+\mathscr{U}+\mathscr{A}_{S} & \leqslant K\left(\|v\|_{\Omega_{t}}^{2}+\left\|\rho-\rho_{S}\right\|_{\Omega_{t}}^{2}+\left\|m-m_{S}\right\|_{C\left(\bar{\Omega}_{t}\right)}^{2}+\left(R-R_{S}\right)^{2}\right) \\
& \leqslant K_{1}\left(\left\|\rho-\rho_{S}\right\|_{\Omega_{t}}^{2}+\|v\|_{\Omega_{t}}^{2}\right) .
\end{aligned}
$$

Более того, аналогично (47)

$$
\begin{aligned}
\int_{\Omega_{t}} \Pi_{1}\left(\rho, \rho_{S}\right)\left(\rho-\rho_{S}\right)^{2} \varkappa d r & =\int_{\Omega_{t}}\left(\Pi_{1}\left(\rho_{S}, \rho_{S}\right)+\alpha\left[\left\|\rho-\rho_{S}\right\|_{C\left(\bar{\Omega}_{t}\right)}\right]\right)\left(\rho-\rho_{S}\right)^{2} \varkappa d r \\
& =\int_{\Omega_{t}} \frac{1}{2} \frac{p^{\prime}\left(\rho_{S}\right)}{\rho_{S}}\left(\rho-\rho_{S}\right)^{2} \varkappa d r+o\left(\left\|\rho-\rho_{S}\right\|_{\Omega_{t}}^{2}\right) .
\end{aligned}
$$

Поскольку $\mathscr{A}_{S}\left(R_{S}, R_{S}\right)=\left.\left(\mathscr{A}_{S}\right)_{r}\left(r, R_{S}\right)\right|_{r=R_{S}}=0$, а также

$$
\left(\mathscr{A}_{S}\right)_{r r}\left(r, R_{S}\right)=-\left(p\left(\rho_{S}\right)\right)_{r} \varkappa+\left(p_{\Gamma, S}-p\left(\rho_{S}\right)\right) \varkappa_{r}
$$

то в силу формулы Тейлора и стационарного уравнения (7) получим

$$
\mathscr{A}_{S}\left(R, R_{S}\right)=-\frac{1}{2}\left(\rho_{S} f_{S}\left[m_{S}\right] \varkappa\right)\left(R_{S}\right)\left(R-R_{S}\right)^{2}+o\left(\left(R-R_{S}\right)^{2}\right) .
$$

В силу равенства $(87)$ также $\left(m-m_{S}\right)_{R}=-\left(\rho_{S} \varkappa\right)\left(R_{S}\right)\left(R-R_{S}\right)+o\left(R-R_{S}\right)$, поэтому

$$
\mathscr{A}_{S}\left(R, R_{S}\right)=-\frac{1}{2} \frac{f_{S}\left[m_{S}\right]}{\rho_{S} \varkappa}\left(R_{S}\right)\left(m-m_{S}\right)_{R}^{2}+o\left(\left(R-R_{S}\right)^{2}\right) .
$$


С учетом неравенств (83) и (53) с $m$ и $R$ в роли $m_{S, n}$ и $R_{S, n}$ это означает, что

$$
\mathscr{U}+\mathscr{A}_{S}=\frac{1}{2} \mathscr{J}_{R}\left[m-m_{S}\right]+o\left(\left\|\rho-\rho_{S}\right\|_{\Omega_{t}}^{2}\right) \geqslant K^{-1}\left\|\rho-\rho_{S}\right\|_{\Omega_{t}}^{2}
$$

при достаточно больших $t \geqslant t_{1}$. Следовательно, справедлива и оценка снизу

$$
K^{-1}\left(\|v\|_{\Omega_{t}}^{2}+\left\|\rho-\rho_{S}\right\|_{\Omega_{t}}^{2}\right) \leqslant \mathscr{E}+\mathscr{U}+\mathscr{A}_{S}
$$

Шаг 2. Разделим уравнение импульса на $\rho$ и вычтем из результата стационарное уравнение (26):

$$
v_{t}+\frac{1}{2}\left(v^{2}\right)_{r}+\pi(\rho)_{r}-\pi\left(\rho_{S}\right)_{r}-\frac{1}{\rho}\left[\frac{\mu(\rho)}{\varkappa}(\varkappa v)_{r}\right]_{r}=f_{S}[m]-f_{S}\left[m_{S}\right]+\Delta f[m] .
$$

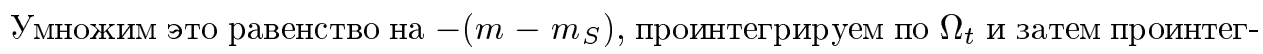
рируем по частям. Воспользовавшись формулами

$$
\begin{aligned}
-\int_{\Omega_{t}} v_{t}\left(m-m_{S}\right) d r & =-\int_{\Omega_{t}}\left(\left(v\left(m-m_{S}\right)\right)_{t}+\rho v^{2} \varkappa\right) d r \\
& =-\frac{d}{d t} \int_{\Omega_{t}} v\left(m-m_{S}\right) d r+\left(v^{2}\left(m-m_{S}\right)\right)_{R}-\int_{\Omega_{t}} \rho v^{2} \varkappa d r
\end{aligned}
$$

и $\left(m-m_{S}\right)_{r}=\left(\rho-\rho_{S}\right) \varkappa$ вместе с условием на свободной границе, выведем

$$
\begin{array}{rl}
-\frac{d}{d t} \int_{\Omega_{t}} & v\left(m-m_{S}\right) d r-\frac{1}{2} \int_{\Omega_{t}}\left(\rho+\rho_{S}\right) v^{2} \varkappa d r \\
& +\int_{\Omega_{t}}\left[\left(\pi(\rho)-\pi\left(\rho_{S}\right)\right)\left(\rho-\rho_{S}\right) \varkappa+\left(f_{S}[m]-f_{S}\left[m_{S}\right]\right)\left(m-m_{S}\right)\right] d r \\
& -\int_{\Omega_{t}} \frac{\mu(\rho)}{\varkappa}(\varkappa v)_{r}\left(\frac{1}{\rho}\left(\rho-\rho_{S}\right) \varkappa-\frac{\rho_{r}}{\rho^{2}}\left(m-m_{S}\right)\right) d r \\
& +\left[\frac{1}{2} v^{2}-\left(\pi(\rho)-\pi\left(\rho_{S}\right)\right)+\frac{\widetilde{\mu}_{1}(\rho) v}{\rho r}+\frac{1}{\rho}\left(p(\rho)-p_{\Gamma}\right)\right]_{R}\left(m-m_{S}\right)_{R} \\
= & -\int_{\Omega_{t}} \Delta f[m]\left(m-m_{S}\right) d r .
\end{array}
$$

Вспомнив обозначение (46) и применив соотношения $(52)$ и $(53)$ с $\rho(\cdot, t), m(\cdot, t)$ и $R(t)$ вместо $\rho_{S, n}, m_{S, n}$ и $R_{S, n}$, выведем также

$$
\begin{aligned}
\int_{\Omega_{t}}[( & \left.\left.\pi(\rho)-\pi\left(\rho_{S}\right)\right)\left(\rho-\rho_{S}\right) \varkappa+\left(f_{S}[m]-f_{S}\left[m_{S}\right]\right)\left(m-m_{S}\right)\right] d r \\
& \quad-\left(\pi(\rho)-\pi\left(\rho_{S}\right)\right)_{R}\left(m-m_{S}\right)_{R} \\
= & \mathscr{B}_{R}\left(\rho, \rho_{S}\right) \geqslant K^{-1}\left\|\rho-\rho_{S}\right\|_{\Omega_{t}}^{2}+o\left(\left\|\rho-\rho_{S}\right\|_{\Omega_{t}}^{2}\right) \geqslant K_{1}^{-1}\left\|\rho-\rho_{S}\right\|_{\Omega_{t}}^{2}
\end{aligned}
$$

при достаточно больших $t \geqslant t_{2}$. 
Оценим последовательно другие интегралы в равенстве (90). Согласно неравенствам (86)

$$
\begin{gathered}
\left|\int_{\Omega_{t}} v\left(m-m_{S}\right) d r\right| \leqslant \bar{R}^{k+1}\left(\|v\|_{\Omega_{t}}^{2}+\left\|\rho-\rho_{S}\right\|_{\Omega_{t}}^{2}\right) \\
\int_{\Omega_{t}}\left(\rho+\rho_{S}\right) v^{2} \varkappa d r \leqslant 3 \bar{\rho} \bar{R}^{k}\|v\|_{\Omega_{t}}^{2}, \\
\left|\int_{\Omega_{t}} \frac{\mu(\rho)}{\varkappa}(\varkappa v)_{r}\left(\frac{1}{\rho}\left(\rho-\rho_{S}\right) \varkappa-\frac{\rho_{r}}{\rho^{2}}\left(m-m_{S}\right)\right) d r\right| \\
\leqslant\|\mu\|_{C[\underline{\rho}, \bar{\rho}]}\left\|(\varkappa v)_{r}\right\|_{\Omega_{t}}\left(\underline{\rho}^{-1}\left\|\rho-\rho_{S}\right\|_{\Omega_{t}}+r_{0}^{-k} \underline{\rho}^{-2}\left\|\rho_{r}\right\|_{\Omega_{t}}\left\|m-m_{S}\right\|_{C\left(\bar{\Omega}_{t}\right)}\right) \\
\leqslant K \varepsilon^{-1}\left\|(\varkappa v)_{r}\right\|_{\Omega_{t}}^{2}+\varepsilon\left\|\rho-\rho_{S}\right\|_{\Omega_{t}}^{2} \quad \forall \varepsilon>0,
\end{gathered}
$$

где использованы (73), а также оценка

$$
\left|\int_{\Omega_{t}} \Delta f[m]\left(m-m_{S}\right) d r\right| \leqslant \bar{R}^{k+1}\|\Delta f[m]\|_{\Omega_{t}}\left\|\rho-\rho_{S}\right\|_{\Omega_{t}} .
$$

Далее, оценим оставшиеся слагаемые граничного члена в (90):

$$
\begin{aligned}
& \left.|| \frac{1}{2} v^{2}+\frac{\widetilde{\mu}_{1}(\rho) v}{\rho r}+\frac{1}{\rho}\left(p(\rho)-p_{\Gamma}\right)\right]_{R}\left(m-m_{S}\right)_{R} \mid \\
& \leqslant \\
& \quad \frac{1}{2} v_{R}^{2}\left(M+m_{S}(R)\right)+\left\{2 k \widehat{\mu}_{1}\left(\underline{\rho} r_{0}\right)^{-1}\left|v_{R}\right|\right. \\
& \left.\quad+\bar{\rho}^{-1}\left(\left\|p^{\prime}\right\|_{C[\underline{\rho}, \bar{\rho}]}\left|\rho_{R}-\rho_{\Gamma, S}\right|+\left|\Delta p_{\Gamma}\right|\right)\right\} \bar{R}^{k+1}\left\|\rho-\rho_{S}\right\|_{\Omega_{t}} \quad \\
& \leqslant \\
& K \varepsilon^{-1}\left(\|v\|_{C\left(\bar{\Omega}_{t}\right)}^{2}+\left(\rho_{R}-\rho_{\Gamma, S}\right)^{2}+\left(\Delta p_{\Gamma}\right)^{2}\right)+\varepsilon\left\|\rho-\rho_{S}\right\|_{\Omega_{t}}^{2} \quad \forall \varepsilon \in(0,1] .
\end{aligned}
$$

Собрав вместе полученные оценки, применив неравенство (84) и выбрав $\varepsilon:=$ $1 / K$ достаточно мальм, в итоге выведем из (90) следуюшее неравенство:

$$
\begin{aligned}
& -\frac{d}{d t} \int_{\Omega_{t}} v\left(m-m_{S}\right) d r+K^{-1}\left\|\rho-\rho_{S}\right\|_{\Omega_{t}}^{2} \\
& \quad \leqslant K\left(\left\|(\varkappa v)_{r}\right\|_{\Omega_{t}}^{2}+\left(\rho_{R}-\rho_{\Gamma, S}\right)^{2}+\left(\Delta p_{\Gamma}\right)^{2}+\|\Delta f[m]\|_{\Omega_{t}}\left\|\rho-\rho_{S}\right\|_{\Omega_{t}}\right) .
\end{aligned}
$$

Шаг 3. Теперь умножим уравнение на свободной границе

$$
\frac{d \rho_{R}}{d t}+\frac{\rho_{R}}{\mu\left(\rho_{R}\right)}\left(p\left(\rho_{R}\right)-p_{\Gamma, S}\right)=b_{1, R}:=\frac{\rho_{R}}{\mu\left(\rho_{R}\right)}\left(-\widetilde{\mu}_{1}\left(\rho_{R}\right) \frac{v_{R}}{R}+\Delta p_{\Gamma}\right)
$$

(см. $\left[10 ;\right.$ доказательство утверждения 2]) на $\rho_{R}-\rho_{\Gamma, S}$ и получим

$$
\frac{1}{2} \frac{d}{d t}\left(\rho_{R}-\rho_{\Gamma, S}\right)^{2}+\frac{\rho_{R}}{\mu\left(\rho_{R}\right)}\left(p\left(\rho_{R}\right)-p_{\Gamma, S}\right)\left(\rho_{R}-\rho_{\Gamma, S}\right)=b_{1, R}\left(\rho_{R}-\rho_{\Gamma, S}\right) .
$$

В силу оценок $\underline{\rho} \leqslant \rho_{R} \leqslant \bar{\rho}$ вместе с условиями (61) на $\mu, \mu_{1}$ и $p^{\prime}>0$ найдем

$$
\frac{d}{d t}\left(\rho_{R}-\rho_{\Gamma, S}\right)^{2}+K^{-1}\left(\rho_{R}-\rho_{\Gamma, S}\right)^{2} \leqslant K\left(\|v\|_{C\left(\bar{\Omega}_{t}\right)}^{2}+\left(\Delta p_{\Gamma}\right)^{2}\right) .
$$


Просуммировав неравенство (85) с неравенствами (92) и (93), умноженньми на параметры $\varepsilon_{1}>0$ и $\varepsilon_{2}>0$, а также применив неравенства (86) и (84), вьведем

$$
\begin{aligned}
\frac{d \mathscr{V}_{0}}{d t}+ & K^{-1}\left\{\left(1-K^{2}\left(\varepsilon_{1}+\varepsilon_{2}\right)\right)\left\|(\varkappa v)_{r}\right\|_{\Omega_{t}}^{2}\right. \\
& \left.+\varepsilon_{1}\left\|\rho-\rho_{S}\right\|_{\Omega_{t}}^{2}+\left(\varepsilon_{2}-K^{2} \varepsilon_{1}\right)\left(\rho_{R}-\rho_{\Gamma, S}\right)^{2}\right\} \\
\leqslant & o\left(\left\|\rho-\rho_{S}\right\|_{\Omega_{t}}^{2}\right)+K\left\{\left(1+\varepsilon_{1}\right)\|\Delta f[m]\|_{\Omega_{t}}\left(\mathscr{E}+\left\|\rho-\rho_{S}\right\|_{\Omega_{t}}^{2}\right)^{1 / 2}\right. \\
& \left.+\left(1+\varepsilon_{1}+\varepsilon_{2}\right)\left(\Delta p_{\Gamma}\right)^{2}\right\}
\end{aligned}
$$

c

$$
\mathscr{V}_{0}:=\mathscr{E}+\mathscr{U}+\mathscr{A}_{S}-\varepsilon_{1} \int_{\Omega_{t}} v\left(m-m_{S}\right) d r+\varepsilon_{2}\left(\rho_{R}-\rho_{\Gamma, S}\right)^{2} .
$$

Последовательно выбрав $\varepsilon_{2}$ и $\varepsilon_{1}$ достаточно малыми и приняв во внимание неравенства $(88),(89)$ и $(91)$, при достаточно больших $t \geqslant t_{0}$ получим

$\frac{d \mathscr{V}_{0}}{d t}+K^{-1}\left\{\left\|(\varkappa v)_{r}\right\|_{\Omega_{t}}^{2}+\left\|\rho-\rho_{S}\right\|_{\Omega_{t}}^{2}+\left(\rho_{R}-\rho_{\Gamma, S}\right)^{2}\right\} \leqslant K\left\{\|\Delta f[m]\|_{\Omega_{t}} \mathscr{V}_{0}^{1 / 2}+\left(\Delta p_{\Gamma}\right)^{2}\right\}$

вместе с

$$
\mathscr{V}_{0} \asymp\|v\|_{\Omega_{t}}^{2}+\left\|\rho-\rho_{S}\right\|_{\Omega_{t}}^{2}+\left(\rho_{R}-\rho_{\Gamma, S}\right)^{2} .
$$

Здесь и ниже символика $\mathscr{V}_{i} \asymp \mathscr{A}_{i}$ означает, что $K^{-1} \mathscr{A}_{i} \leqslant \mathscr{V}_{i} \leqslant K \mathscr{A}_{i}$. Ясно, что эти соотношения влекут, что $\mathscr{V}_{0}$ является функционалом Ляпунова и справедлива первая оценка скорости стабилизации (79).

Шаг 4. Чтобы доказать оценку (80), сначала напомним, что при выполнении условий (17) из утверждения 4 следует, что $\left\|\left(\rho_{S}\right)_{r r}\right\|_{\Omega_{t}} \leqslant K$. Перепишем уравнение импульса в форме

$$
\rho \varkappa D_{t}\left(\varkappa^{-1}(d[\rho]+v)\right)+p(\rho)_{r}=-\varkappa^{-1} \varkappa_{r} \rho v^{2}+\rho f[m]
$$

с $d[\rho]:=\left(\mu(\rho) / \rho^{2}\right) \rho_{r}$ (см. [10; доказательство теоремы 5, п. 2]) и, воспользовавшись стационарным уравнением, получим

$$
\begin{aligned}
& \rho \varkappa D_{t}\left(\varkappa^{-1}\left(d[\rho]-d\left[\rho_{S}\right]+v\right)\right)+\left(p(\rho)-p\left(\rho_{S}\right)\right)_{r} \\
& \quad=-\rho \varkappa v\left(\varkappa^{-1} d\left[\rho_{S}\right]\right)_{r}-k^{-1} r \rho v^{2}+\rho f_{S}[m]-\rho_{S} f_{S}\left[m_{S}\right]+\rho \Delta f[m] .
\end{aligned}
$$

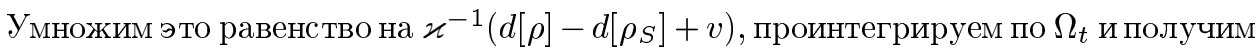
(см. также формулу (63))

$$
\begin{aligned}
& \frac{d \mathscr{Y}}{d t}+\int_{\Omega_{t}}\left(p(\rho)-p\left(\rho_{S}\right)\right)_{r}\left(d[\rho]-d\left[\rho_{S}\right]\right) \varkappa^{-1} d r \\
&=b_{2}:=\int_{\Omega_{t}}\left\{-\left(p(\rho)-p\left(\rho_{S}\right)\right)_{r} v \varkappa^{-1}+\left(-\rho \varkappa v\left(\varkappa^{-1} d\left[\rho_{S}\right]\right)_{r}-k^{-1} r \rho v^{2}\right.\right. \\
&+\left(\rho-\rho_{S}\right) f_{S}[m]+\rho_{S}\left(f_{S}[m]-f_{S}\left[m_{S}\right]\right) \\
&\left.+\rho \Delta f[m])\left(d[\rho]-d\left[\rho_{S}\right]+v\right)\right\} \varkappa^{-1} d r
\end{aligned}
$$

c

$$
\mathscr{Y}:=\frac{1}{2} \int_{\Omega_{t}} \rho\left(d[\rho]-d\left[\rho_{S}\right]+v\right)^{2} \varkappa^{-1} d r
$$


Ясно, что

$$
\begin{aligned}
d[\rho]-d\left[\rho_{S}\right] & =\frac{\mu(\rho)}{\rho^{2}}\left(\rho-\rho_{S}\right)_{r}+\left(\frac{\mu(\rho)}{\rho^{2}}-\frac{\mu\left(\rho_{S}\right)}{\rho_{S}^{2}}\right)\left(\rho_{S}\right)_{r} \\
\left(p(\rho)-p\left(\rho_{S}\right)\right)_{r} & =p^{\prime}(\rho)\left(\rho-\rho_{S}\right)_{r}+\left(p^{\prime}(\rho)-p^{\prime}\left(\rho_{S}\right)\right)\left(\rho_{S}\right)_{r} .
\end{aligned}
$$

Применив условия на $\mu, p$ и $f_{S}$ (включая (17)) вместе с оценками $\underline{\rho} \leqslant \rho \leqslant \bar{\rho}$, $\delta_{S} / 2 \leqslant \rho_{S} \leqslant 2 \bar{\rho}, r_{0} \leqslant r \leqslant \bar{R}$ и (73), можно вывести оценки (ср. с [21])

$$
\begin{gathered}
K^{-1}\left\|\left(\rho-\rho_{S}\right)_{r}\right\|_{\Omega_{t}}^{2}-K\left(\left\|\rho-\rho_{S}\right\|_{\Omega_{t}}^{2}+\|v\|_{\Omega_{t}}^{2}\right) \leqslant \mathscr{Y} \leqslant K\left(\left\|\rho-\rho_{S}\right\|_{H^{1}\left(\Omega_{t}\right)}^{2}+\|v\|_{\Omega_{t}}^{2}\right), \\
(98)
\end{gathered}
$$

а также

$$
\begin{aligned}
\left|b_{2}\right| \leqslant & K\left\{\left\|\rho-\rho_{S}\right\|_{H^{1}\left(\Omega_{t}\right)}\|v\|_{\Omega_{t}}+\left(\|\varkappa v\|_{C\left(\bar{\Omega}_{t}\right)}+\|\varkappa v\|_{C\left(\bar{\Omega}_{t}\right)}\|\sqrt{\rho} \varkappa v\|_{\Omega_{t}}\right.\right. \\
& \left.\left.+\left\|\rho-\rho_{S}\right\|_{\Omega_{t}}+\|\|\left(f_{S}\right)_{\chi}\left\|_{C\left[0, M_{1}\right]}\right\|_{\Omega_{t}}\left\|m-m_{S}\right\|_{C\left(\bar{\Omega}_{t}\right)}+\|\Delta f[m]\|_{\Omega_{t}}\right) \mathscr{Y}^{1 / 2}\right\} .
\end{aligned}
$$

Следовательно,

$$
\begin{gathered}
\left|b_{2}\right| \leqslant \varepsilon\left\|\left(\rho-\rho_{S}\right)_{r}\right\|_{\Omega_{t}}^{2}+K \varepsilon^{-1}\left(\left\|(\varkappa v)_{r}\right\|_{\Omega_{t}}^{2}+\left\|\rho-\rho_{S}\right\|_{\Omega_{t}}^{2}\right)+K\|\Delta f[m]\|_{\Omega_{t}} \mathscr{Y}^{1 / 2} \\
\forall \varepsilon \in(0,1]
\end{gathered}
$$

и равенство (96) вместе с (99) влекут

$$
\frac{d \mathscr{Y}}{d t}+K^{-1}\left\|\left(\rho-\rho_{S}\right)_{r}\right\|_{\Omega_{t}}^{2} \leqslant K\left(\left\|(\varkappa v)_{r}\right\|_{\Omega_{t}}^{2}+\left\|\rho-\rho_{S}\right\|_{\Omega_{t}}^{2}+\|\Delta f[m]\|_{\Omega_{t}} \mathscr{Y}^{1 / 2}\right) .
$$

Скомбинировав это неравенство с (94) и применив соотношения (95) и (98), для $\mathscr{V}_{1}:=\mathscr{V}_{0}+\varepsilon_{3} \mathscr{Y}$ при подходящем $\varepsilon_{3}>0$ выведем

$$
\begin{aligned}
\frac{d \mathscr{V}_{1}}{d t}+ & K^{-1}\left\{\left\|(\varkappa v)_{r}\right\|_{\Omega_{t}}^{2}+\left\|\rho-\rho_{S}\right\|_{H^{1}\left(\Omega_{t}\right)}^{2}+\left(\rho_{R}-\rho_{\Gamma, S}\right)^{2}\right\} \\
& \leqslant K\left\{\|\Delta f[m]\|_{\Omega_{t}} \mathscr{V}_{1}^{1 / 2}+\left(\Delta p_{\Gamma}\right)^{2}\right\}
\end{aligned}
$$

вместе с

$$
\mathscr{V}_{1} \asymp\|v\|_{\Omega_{t}}^{2}+\left\|\rho-\rho_{S}\right\|_{H^{1}\left(\Omega_{t}\right)}^{2}+\left(\rho_{R}-\rho_{\Gamma, S}\right)^{2} .
$$

Таким образом, $\mathscr{V}_{1}$ является нашим вторым функционалом Ляпунова и верна оценка (80).

ШШаг 5. Пусть теперь выполнены оба условия (75) и (17). Напомним уравнение импульса в следуюшей форме (см. [10; доказательство теоремы 5 , п. 3]):

$$
\varkappa^{-1} D_{t}(\varkappa v)=\frac{1}{\rho} \sigma_{r}+k \frac{v^{2}}{r}+f[m]=\mu(\rho) \Psi_{r}+b_{3}
$$


с функциями

$$
\begin{aligned}
\sigma & :=\frac{\mu(\rho)}{\varkappa}(\varkappa v)_{r}-\widetilde{\mu}_{1}(\rho) \frac{v}{r}-p(\rho), \quad \Psi:=\frac{\sigma+p_{\Gamma}}{\rho \mu(\rho)} \\
b_{3} & :=\frac{1}{\rho}(\rho \mu(\rho))_{r} \Psi+\frac{k}{r} v^{2}+\frac{1}{\rho}\left(\widetilde{\mu}_{1}(\rho) \frac{v}{r}\right)_{r}+f[m] .
\end{aligned}
$$

Перепишем это уравнение с использованием стационарного уравнения так:

$$
\varkappa^{-1} D_{t}(\varkappa v)=\mu(\rho) \Phi_{r}+\widetilde{b}_{3}
$$

где

$$
\begin{array}{r}
\Phi:=\frac{\sigma+p\left(\rho_{S}\right)+\Delta p_{\Gamma}}{\rho \mu(\rho)}=\frac{1}{\rho \varkappa}(\varkappa v)_{r}-\omega_{2}(\rho) \frac{v}{r}-\frac{p(\rho)-p\left(\rho_{S}\right)-\Delta p_{\Gamma}}{\rho \mu(\rho)}, \quad(103) \\
\omega_{2}(\rho):=\frac{\widetilde{\mu}_{1}(\rho)}{\rho \mu(\rho)}, \quad \widetilde{b}_{3}:=\frac{1}{\rho}(\rho \mu(\rho))_{r} \Phi+\frac{k}{r} v^{2}+\frac{1}{\rho}\left(\widetilde{\mu}_{1}(\rho) \frac{v}{r}\right)_{r}+f[m]-\frac{\rho_{S}}{\rho} f_{S}\left[m_{S}\right] .
\end{array}
$$

Заметим, что $\Phi_{R}=0$ и

$$
\begin{aligned}
& \bar{\rho}^{-1}\left\|v_{r}\right\|_{\Omega_{t}}-K\left(\|v\|_{\Omega_{t}}+\left\|\rho-\rho_{S}\right\|_{\Omega_{t}}+\left|\Delta p_{\Gamma}\right|\right) \\
& \leqslant\|\Phi\|_{\Omega_{t}} \leqslant K\left(\left\|v_{r}\right\|_{\Omega_{t}}+\left\|\rho-\rho_{S}\right\|_{\Omega_{t}}+\left|\Delta p_{\Gamma}\right|\right) .
\end{aligned}
$$

Аналогично соответствуюшим соотношениям для $\Psi$ и $b_{3}$ в [10] справедливы равенство

$$
\begin{aligned}
& \frac{1}{2} \frac{d}{d t} \int_{\Omega_{t}} \rho \Phi^{2} \varkappa d r+\int_{\Omega_{t}} \mu(\rho) \Phi_{r}^{2} \varkappa d r \\
& \quad=-\int_{\Omega_{t}}\left\{\rho\left(D_{t}\left(\omega_{2}(\rho) \frac{v}{r}\right)+D_{t} \frac{p(\rho)-p\left(\rho_{S}\right)-\Delta p_{\Gamma}}{\rho \mu(\rho)}\right) \Phi+\widetilde{b}_{3} \Phi_{r}\right\} \varkappa d r
\end{aligned}
$$

и оценки

$$
\begin{aligned}
\left|D_{t}\left(\omega_{2}(\rho) \frac{v}{r}\right)\right| \leqslant & K\left(\left|(\varkappa v)_{r} \sqrt{\rho \varkappa} v\right|+\left|\mu(\rho) \Phi_{r}+\widetilde{b}_{3}-(k+1) \frac{v^{2}}{r}\right|\right) \\
\leqslant & K_{1}\left(\left|(\varkappa v)_{r}\right|+\left|\Phi_{r}\right|+\left|\widetilde{b}_{3}\right|+|\varkappa v|\right), \\
\left\|\widetilde{b}_{3}\right\|_{\Omega_{t}} \leqslant & \varepsilon\left\|\Phi_{r}\right\|_{\Omega_{t}}+K\left(\varepsilon^{-1}\|\Phi\|_{\Omega_{t}}+\left\|(\varkappa v)_{r}\right\|_{\Omega_{t}}\right) \\
& +\left\|f[m]-\frac{\rho_{S}}{\rho} f_{S}\left[m_{S}\right]\right\|_{\Omega_{t}} \quad \forall \varepsilon>0
\end{aligned}
$$

с $\Phi$ и $\widetilde{b}_{3}$; дополнительно в (107) была применена оценка $\|v\|_{C_{b}(\bar{Q})} \leqslant K$. Положив $\omega(\rho):=1 /(\rho \mu(\rho))$ и воспользовавшись уравнением неразрывности, имеем также

$$
\begin{aligned}
&\left|D_{t}\left[\omega(\rho)\left(p(\rho)-p\left(\rho_{S}\right)-\Delta p_{\Gamma}\right)\right]\right| \\
&=\mid \omega(\rho)\left(-p^{\prime}(\rho) \varkappa^{-1}(\varkappa \rho v)_{r}-p_{\Gamma}^{\prime}+v\left(p(\rho)-p\left(\rho_{S}\right)\right)_{r}\right) \\
& \quad-\rho \omega^{\prime}(\rho) \varkappa^{-1}(\varkappa v)_{r}\left(p(\rho)-p\left(\rho_{S}\right)-\Delta p_{\Gamma}\right) \mid \\
& \leqslant K\left\{\left|\rho_{r}\right||\varkappa v|+\left|(\varkappa v)_{r}\right|+\left|p_{\Gamma}^{\prime}\right|+\left|\left(p(\rho)-p\left(\rho_{S}\right)\right)_{r}\right|\right. \\
&\left.+\left|(\varkappa v)_{r}\right|\left(\left|p(\rho)-p\left(\rho_{S}\right)\right|+\left|\Delta p_{\Gamma}\right|\right)\right\} .
\end{aligned}
$$


Применив также равномерные $H^{1}\left(\Omega_{t}\right)$-оценки $(73)$ и $(76)$, из (106) вьведем

$$
\begin{aligned}
\frac{1}{2} \frac{d}{d t}\|\sqrt{\rho \varkappa} \Phi\|_{\Omega_{t}}^{2}+\underline{\mu}\left\|\sqrt{\varkappa} \Phi_{r}\right\|_{\Omega_{t}}^{2} \leqslant K\left\{\left(\left\|(\varkappa v)_{r}\right\|_{\Omega_{t}}+\left\|\Phi_{r}\right\|_{\Omega_{t}}\right.\right. \\
\left.\left.+\left\|p(\rho)-p\left(\rho_{S}\right)\right\|_{H^{1}\left(\Omega_{t}\right)}+\left|p_{\Gamma}^{\prime}\right|+\left|\Delta p_{\Gamma}\right|\right)\|\Phi\|_{\Omega_{t}}+\left\|\widetilde{b}_{3}\right\|_{\Omega_{t}}\left\|\Phi_{r}\right\|_{\Omega_{t}}\right\} .
\end{aligned}
$$

Применив, во-первых, равенство (97) и оценку (108) вместе с соотношениями

$$
f[m]=f_{S}[m]+\Delta f[m]
$$

и

$$
\begin{aligned}
& \left\|f_{S}[m]-\frac{\rho_{S}}{\rho} f_{S}\left[m_{S}\right]\right\|_{\Omega_{t}}=\left\|\frac{1}{\rho}\left(\rho-\rho_{S}\right) f_{S}\left[m_{S}\right]+f_{S}[m]-f_{S}\left[m_{S}\right]\right\|_{\Omega_{t}} \\
& \quad \leqslant K\left\|\rho-\rho_{S}\right\|_{\Omega_{t}}+\|\|\left(f_{S}\right)_{\chi}\left\|_{C\left[0, M_{1}\right]}\right\|_{\Omega_{t}}\left\|m-m_{S}\right\|_{C\left(\bar{\Omega}_{t}\right)} \leqslant K_{1}\left\|\rho-\rho_{S}\right\|_{\Omega_{t}}
\end{aligned}
$$

и, во-вторых, правое неравенство (105), установим неравенства

$$
\begin{aligned}
& \frac{d}{d t}\|\sqrt{\rho \varkappa} \Phi\|_{\Omega_{t}}^{2}+\underline{\mu}\left\|\sqrt{\varkappa} \Phi_{r}\right\|_{\Omega_{t}}^{2} \\
& \leqslant K\left(\|\Phi\|_{\Omega_{t}}^{2}+\left\|(\varkappa v)_{r}\right\|_{\Omega_{t}}^{2}+\left|p_{\Gamma}^{\prime}\right|\|\Phi\|_{\Omega_{t}}+\left\|\rho-\rho_{S}\right\|_{H^{1}\left(\Omega_{t}\right)}^{2}\right. \\
&\left.\quad+\left(\Delta p_{\Gamma}\right)^{2}+\|\Delta f[m]\|_{\Omega_{t}}^{2}\right) \\
& \leqslant K_{1}\left(\left\|v_{r}\right\|_{\Omega_{t}}^{2}+\left\|\rho-\rho_{S}\right\|_{H^{1}\left(\Omega_{t}\right)}^{2}+\|\Delta f[m]\|_{\Omega_{t}}^{2}+\left(\Delta p_{\Gamma}\right)^{2}+\left|p_{\Gamma}^{\prime}\right|\|\sqrt{\rho \varkappa} \Phi\|_{\Omega_{t}}\right) .
\end{aligned}
$$

Скомбинировав их с (100) и очевидным тождеством

$$
\frac{1}{2} \frac{d}{d t}\left(\Delta p_{\Gamma}\right)^{2}+\delta\left(\Delta p_{\Gamma}\right)^{2}=\delta\left(\Delta p_{\Gamma}\right)^{2}+p_{\Gamma}^{\prime} \Delta p_{\Gamma} \quad \forall \delta,
$$

а также воспользовавшись $(101)$ и (105), для $\mathscr{V}_{2}:=\mathscr{V}_{1}+\varepsilon_{4}\|\sqrt{\rho \varkappa} \Phi\|_{\Omega_{t}}^{2}+\left(\Delta p_{\Gamma}\right)^{2}$ при подходяшем $\varepsilon_{4}>0$ выведем

$$
\begin{gathered}
\frac{d \mathscr{V}_{2}}{d t}+K^{-1}\left\{\|v\|_{H^{1}\left(\Omega_{t}\right)}^{2}+\left\|\rho-\rho_{S}\right\|_{H^{1}\left(\Omega_{t}\right)}^{2}+\left(\rho_{R}-\rho_{\Gamma, S}\right)^{2}+\left(\Delta p_{\Gamma}\right)^{2}+\left\|\Phi_{r}\right\|_{\Omega_{t}}^{2}\right\} \\
\leqslant K\left\{\|\Delta f[m]\|_{\Omega_{t}}^{2}+\left(\Delta p_{\Gamma}\right)^{2}+\left|p_{\Gamma}^{\prime}\right| \mathscr{V}_{2}^{1 / 2}\right\}
\end{gathered}
$$

вместе с

$$
\mathscr{V}_{2} \asymp\|v\|_{H^{1}\left(\Omega_{t}\right)}^{2}+\left\|\rho-\rho_{S}\right\|_{H^{1}\left(\Omega_{t}\right)}^{2}+\left(\rho_{R}-\rho_{\Gamma, S}\right)^{2}+\left(\Delta p_{\Gamma}\right)^{2} .
$$

Тем самым справедлива последняя оценка (81). Отметим, что $\mathscr{V}_{2}$ также является функционалом Ляпунова при условии, что $\Delta p_{\Gamma}=0$. Пункт 1) доказан.

Шаг 6. Дадим обоснование п. 2). В соответствии с доказательством п. 1) все оценки скорости стабилизации справедливы при $0 \leqslant t \leqslant T$ с любым $T \geqslant 0$ при следуюших априорных оценках решения:

$$
\max _{[0, T]}\left|R(t)-R_{S}\right| \leqslant \varepsilon_{S}, \quad \max _{[0, T]}\left\|\rho(\cdot, t)-\rho_{S}(\cdot)\right\|_{C\left(\bar{\Omega}_{t}\right)} \leqslant \varepsilon_{1}
$$

с достаточно малым $\varepsilon_{1}>0$, а также

$$
0<\underline{s}_{0} \leqslant \rho(r, t) \leqslant \bar{s}_{0} \text { на } \bar{Q}_{T}, \quad \max _{[0, T]}\left\|\rho_{r}(\cdot, t)\right\|_{L^{2}\left(\Omega_{t}\right)} \leqslant N_{1} .
$$


Важньй момент заключается в том, что величины $K$ и $a_{i}$ в этих оценках зависят от $\varepsilon_{S}, \varepsilon_{1}, \underline{s}_{0}, \bar{s}_{0}$ и $N_{1}$, но не от $T$.

Ясно, что оценки (113) влекут двусторонние оценки для $\rho$ в (114) с

$$
\underline{s}_{0}:=\min _{\left[r_{0}, R_{S}+\varepsilon_{S}\right]} \rho_{S}-\varepsilon_{1}>0 \quad \text { и } \quad \bar{s}_{0}:=\max _{\left[r_{0}, R_{S}+\varepsilon_{S}\right]} \rho_{S}+\varepsilon_{1}
$$

с достаточно малым $\varepsilon_{1}$. (В случае, когда $p$ является немонотонной, но $p^{\prime}(s)>0$ на $\left(s_{1}, s_{2}\right)$ и $s_{1}<\rho_{S}(r)<s_{2}$ на $\bar{\Omega}_{S}$, можно предположить, что $\varepsilon_{S}$ и $\varepsilon_{1}$ в $(113)$ столь малы, что $s_{1}+\varepsilon_{1} \leqslant \rho_{S}(r) \leqslant s_{2}-\varepsilon_{1}$ на $\left[r_{0}, R_{S}+\varepsilon_{S}\right]$ и поэтому $s_{1}<\underline{s}_{0}<\bar{s}_{0}<s_{2}$.) Далее, согласно [10; доказательство оценки (73) в теореме 5] вторая оценка в (114) также верна с некоторьм $N_{1}$, зависящим от $\varepsilon_{S}$ и $\varepsilon_{1}$, но не от $T$.

При выполнении условия (82) с каким-либо $\varepsilon_{0}>0$ оценка скорости стабилизации (79) при $0 \leqslant t \leqslant T$ вместе с замечанием 6 гарантируют выполнение неравенств

$$
\max _{[0, T]}\left|R(t)-R_{S}\right| \leqslant K_{1} \max _{[0, T]}\left\|\rho(\cdot, t)-\rho_{S}(\cdot)\right\|_{\Omega_{t}} \leqslant K_{1} K_{2} \varepsilon_{0}
$$

Следовательно, в силу оценки (43) для $\rho_{S}$ и второй оценки (114)

$$
\max _{[0, T]}\left\|\rho(\cdot, t)-\rho_{S}(\cdot)\right\|_{C\left(\bar{\Omega}_{t}\right)} \leqslant K_{3}\left(K_{2} \varepsilon_{0}\right)^{1 / 2}
$$

Величины $K_{i}, i=1,2,3$, не зависят от $T$ (и $\left.\varepsilon_{0}\right)$; можно считать, что $K_{i} \geqslant 1$. Выберем $\varepsilon_{0}$ так, чтобы $2 K_{1} K_{2} \varepsilon_{0} \leqslant \varepsilon_{S}$ и $2 K_{3}\left(K_{2} \varepsilon_{0}\right)^{1 / 2} \leqslant \varepsilon_{1}$. С помощью стандартных рассуждений о продолжении, основанных на непрерьвности $\rho, R$ и $\rho_{S}$, получим последовательно оценки (113) и (115), (116) и, таким образом, снова (113) с $\varepsilon_{S}$ и $\varepsilon_{1}$, замененньми на $\varepsilon_{S} / 2$ и $\varepsilon_{1} / 2$, сначала для достаточно малых $T=T\left(\varepsilon_{S}, \varepsilon_{1}, \varepsilon_{0}\right)>0$, а в итоге для любых $T>0$. Следовательно, все оценки скорости стабилизации, перечисленные в п. 1), справедливы при всех $t \geqslant 0$.

СлЕДСТВИЕ 2. В условиях теоремы 2, п. 1) или п. 2) (включая условия (17) $u(75)$ в обоих случаях) справедлива оченка $\|v\|_{H^{2,1}(Q)} \leqslant K$.

ДокАЗАТЕльство. Положим $Q^{t_{0}}:=\left\{(r, t) \in Q: t>t_{0}\right\}$ и $\|\varphi\|_{H^{1,0}\left(Q^{\left.t_{0}\right)}\right.}:=$ $\|\varphi\|_{Q^{t_{0}}}+\left\|\varphi_{r}\right\|_{Q^{t_{0}}}$. Проинтегрировав $(111)$ по $\left(t_{0}, \infty\right)$ и применив $(112)$, получим

$$
\begin{aligned}
& \|v\|_{H^{1,0}\left(Q^{t_{0}}\right)}^{2}+\left\|\rho-\rho_{S}\right\|_{H^{1,0}\left(Q^{\left.t_{0}\right)}\right.}^{2}+\|\Phi\|_{H^{1,0}\left(Q^{\left.t_{0}\right)}\right.}^{2} \\
& \quad \leqslant K\left(\mathscr{V}_{2}\left(t_{0}\right)+\|\Delta f[m]\|_{\left(t_{0}, \infty\right)}^{2}+\left\|\Delta p_{\Gamma}\right\|_{\left(t_{0}, \infty\right)}^{2}+\left\|p_{\Gamma}^{\prime}\right\|_{\left(t_{0}, \infty\right)}^{2}\right) \leqslant K_{1} .
\end{aligned}
$$

Далее, из (108) и (109) следует, что $\left\|\widetilde{b}_{3}\right\|_{Q^{t_{0}}} \leqslant K$. Следовательно, уравнение (102) влечет

$$
\left\|v_{t}\right\|_{Q^{t_{0}}}=\left\|-\varkappa^{-1} v(\varkappa v)_{r}+\mu(\rho) \Phi_{r}+\widetilde{b}_{3}\right\|_{Q^{t_{0}}} \leqslant K
$$

В силу равенства (103), умноженного на $\rho$ и продифференцированного по $r$, имеem

$$
\begin{aligned}
\frac{1}{\varkappa}(\varkappa v)_{r r}= & \frac{k}{r \varkappa}(\varkappa v)_{r}+\rho \Phi_{r}+\rho_{r} \Phi+\left(\rho \omega_{2}(\rho)\right)_{r} \frac{v}{r}+\rho \omega_{2}(\rho)\left(\frac{v}{r}\right)_{r} \\
& +\left(\frac{1}{\mu(\rho)}\right)_{r}\left(p(\rho)-p\left(\rho_{S}\right)-\Delta p_{\Gamma}\right)+\frac{1}{\mu(\rho)}\left(p(\rho)-p\left(\rho_{S}\right)\right)_{r} .
\end{aligned}
$$


Применив неравенство $\|\varphi\|_{L^{\infty, 2}\left(Q^{\left.t_{0}\right)}\right.} \leqslant c\|\varphi\|_{H^{1,0}\left(Q^{\left.t_{0}\right)}\right.}$ и равенство $(97)$ вместе с оценками (73) и (117), выведем

$$
\begin{aligned}
\left\|(\varkappa v)_{r r}\right\|_{Q^{t_{0}}} \leqslant & K\left(1+\left\|\rho_{r}\right\|_{L^{2, \infty}(Q)}\right)\left(\|v\|_{H^{1,0}\left(Q^{t_{0}}\right)}+\|\Phi\|_{H^{1,0}\left(Q^{t_{0}}\right)}\right. \\
& \left.+\left\|\rho-\rho_{S}\right\|_{H^{1,0}\left(Q^{t_{0}}\right)}+\left\|\Delta p_{\Gamma}\right\|_{L^{2}\left(t_{0}, \infty\right)}\right) \leqslant K_{1} .
\end{aligned}
$$

Следовательно, и $\left\|v_{r r}\right\|_{Q^{t_{0}}} \leqslant K$.

Аналогичные рассуждения позволяют извлечь неравномерную оценку $\left\|v_{t}\right\|_{Q_{T}}+$ $\left\|v_{r r}\right\|_{Q_{T}} \leqslant K(T)$ при любых $T>0$ из [10; доказательство теоремы 5 , п. 3] (т.е. из доказательства оценки (76) настояшей работы). Следовательно, $\left\|v_{t}\right\|_{Q}+$ $\left\|v_{r r}\right\|_{Q} \leqslant K$. Поскольку справедливы также оценки (71) и (76), то доказательство завершено.

Отметим, что условие на $p_{\Gamma}^{\prime}$ в (75) может быть существенно обобщено (см. [17]).

\section{§4. Лагранжева стационарная задача}

Лагранжева стационарная задача (в фиксированной области $J=(0, M))$ может быть выведена из эйлеровой (7)-(9) в случае положительных решений заменой переменной $\chi=m_{S}(r)$ :

$$
\begin{gathered}
D p\left(\bar{\rho}_{S}\right)=\frac{\check{f}_{S}\left[r_{S}\right]}{r_{S}^{k}}, \quad V_{S}:=\frac{1}{k+1} r_{S}^{k+1}=V_{0}+\int_{0}^{\chi} \eta_{S}(\xi) d \xi, \quad \eta_{S}:=\frac{1}{\bar{\rho}_{S}} \quad \text { в } J, \\
p\left(\bar{\rho}_{S}(M)\right)=p_{\Gamma, S},
\end{gathered}
$$

где неизвестная функция $\bar{\rho}_{S} \in C(\bar{J})$ с $p\left(\bar{\rho}_{S}\right) \in C^{1}(\bar{J})$ и $\left(\bar{\rho}_{S}\right)_{\min }:=\min \bar{J}_{\bar{\rho}}>0$ является положительньм лагранжевым стационарным решением таким, что $\rho_{S}(r)=$ $\bar{\rho}_{S}\left(m_{S}(r)\right)$ при $r \in \bar{\Omega}_{S} ;$ кроме того, здесь

$$
D=\frac{d}{d \chi}, \quad \check{f}_{S}\left[r_{S}\right](\chi)=f_{S}\left(r_{S}(\chi), \chi\right), \quad r_{S} \geqslant r_{0}, \quad V_{0}=\frac{1}{k+1} r_{0}^{k+1} .
$$

Наоборот, если дано положительное решение $\bar{\rho}_{S}$ лагранжевой задачи (118), (119), то обратная замена переменной $r=r_{S}(\chi)$ приводит к положительному решению $\left\{\rho_{S}, R_{S}\right\}$ эйлеровой задачи $(7)-(9)$, где $\rho_{S}\left(r_{S}(\chi)\right)=\bar{\rho}_{S}(\chi)$ при $\chi \in \bar{J}_{\text {и }}$ $R_{S}=r_{S}(M)$. Поэтому сушествует взаимно однозначное соответствие между положительными решениями этих двух задач.

Удобно переписать задачу (118), (119) относительно неизвестной функции $\eta_{S}$ как

$$
\begin{aligned}
D \widehat{p}\left(\eta_{S}\right)=\check{h}\left[V_{S}\right], \quad V_{S}(\chi) & =V_{0}+\int_{0}^{\chi} \eta_{S}(\xi) d \xi \text { в } J, \\
\widehat{p}\left(\eta_{S}(M)\right) & =p_{\Gamma, S}
\end{aligned}
$$

где $\widehat{p}(\zeta)=p(1 / \zeta)$ и $\breve{h}\left[V_{S}\right](\chi):=h\left(V_{S}(\chi), \chi\right)($ см. $(55))$.

Приведем сначала иное доказательство существования положительного решения, данного в замечании 1. Для стационарной задачи в форме (120), (121) с функцией $h$, не зависящей от $\chi$, результат следует из [17], [24]. Воспользуемся теми же рассуждениями, немного упростив их. 
Введем нелинейный оператор $\mathscr{A}: \mathscr{K}^{+} \rightarrow C^{1}(\bar{J})$, где $\mathscr{K}^{+}:=\left\{p_{S} \in C(\bar{J}):\right.$ $\left.\left(p_{S}\right)_{\min }>0\right\}$, положив

$$
\begin{gathered}
\left(\mathscr{A} p_{S}\right)(\chi):=p_{\Gamma, S}-\int_{\chi}^{M} \check{h}\left[V_{S}\right](\xi) d \xi \\
\text { с } V_{S}(\chi)=V_{0}+\int_{0}^{\chi} \widehat{p}^{-1}\left(p_{S}(\xi)\right) d \xi \text { при } \chi \in \bar{J},
\end{gathered}
$$

где $\widehat{p}^{-1}$ - обратная функция к $\widehat{p}$, и переформулируем задачу $(120),(121)$ как задачу о неподвижной точке

$$
p_{S}=\mathscr{A} p_{S}
$$

относительно функции $p_{S}:=\widehat{p}\left(\eta_{S}\right)$. Можно непосредственно проверить, что $\mathscr{A}-$ компактный оператор на $\mathscr{K}_{\delta}^{+}:=\left\{p_{S} \in C(\bar{J}):\left(p_{S}\right)_{\min } \geqslant \delta\right\}$ при любых $\delta>0$.

В силу условий (22) и (13) имеем

$$
\left(\mathscr{A} p_{S}\right)(\chi) \geqslant p_{\Gamma, S}-f_{0}(M-\chi) \geqslant N^{-1} \quad \text { на } \bar{J} .
$$

Кроме того, если $\left(p_{S}\right)_{\min } \geqslant N^{-1}$, то $V_{S} \leqslant \bar{V}_{S}:=V_{0}+M \widehat{p}^{-1}\left(N^{-1}\right)$ и, следовательно,

$$
\left(\mathscr{A} p_{S}\right)(\chi) \leqslant p_{\Gamma, S}+N_{0}(M-\chi) \leqslant p_{\Gamma, S}+N_{0} M \quad \text { на } \bar{J}
$$

с $N_{0}:=\|h\|_{C\left(\left[V_{0}, \bar{V}_{S}\right] \times \bar{J}\right)}$. Это означает, что

$$
\mathscr{A} \mathscr{K} \subset \mathscr{K}
$$

для

$\mathscr{K}:=\left\{p_{S} \in C(\bar{J}): p_{\Gamma, S}-f_{0}(M-\chi) \leqslant p_{S}(\chi) \leqslant p_{\Gamma, S}+N_{0}(M-\chi)\right.$ на $\left.\bar{J}\right\} \subset \mathscr{K}_{N^{-1}}$.

Поскольку $\mathscr{K}$ - вьпуклое замкнутое ограниченное непустое подмножество в $C(\bar{J})$, то задача (122) имеет решение $p_{S} \in \mathscr{K}$ согласно теореме Шаудера о неподвижной точке.

Сформулируем аналог утверждения 2. Пусть $\bar{V}_{S}(\alpha):=\bar{R}_{S}^{k+1}(\alpha) /(k+1)=V_{0}+$ $M / \alpha$ (см. замечание 2).

УТВЕРЖДЕНИЕ 5. 1) Если $h(\nu, \chi)$ не возрастает по $\nu \geqslant V_{0}$ при всех $\chi \in \bar{J}$, то лагранжева стачионарная задача имеет не более одного решения.

2) Пусть $\delta_{S}>0$ и һ удовлетворяет условию

$$
h(\widetilde{\nu}, \chi)-h(\nu, \chi) \leqslant \varphi(\chi)(\widetilde{\nu}-\nu) \quad \text { nри всех } \quad V_{0} \leqslant \nu<\widetilde{\nu} \leqslant \bar{V}_{S}\left(\delta_{S}\right), \quad \chi \in \bar{J}
$$

с некоторой функцией $\varphi \in L^{1}(J), \varphi \geqslant 0$, такой, что

$$
\frac{M}{C^{(2)}\left(\delta_{S}\right)} \int_{J} \varphi d \chi \leqslant q \quad \text { при некотором } 0 \leqslant q \leqslant 1,
$$

где $C^{(2)}\left(\delta_{S}\right):=\inf _{\zeta \leqslant \delta_{S}^{-1}}\left(-\widehat{p}^{\prime}(\zeta)\right)=\inf _{s \geqslant \delta_{S}} s^{2} p^{\prime}(s)>0$. Тогда справедливо неравенство

$$
\begin{aligned}
\widetilde{\mathscr{B}}\left(\eta_{1}, \eta_{2}\right) & :=\int_{J}\left\{\left(\widehat{p}\left(\eta_{2}\right)-\widehat{p}\left(\eta_{1}\right)\right)\left(\eta_{1}-\eta_{2}\right)+\left(\check{h}\left[V_{2}\right]-\check{h}\left[V_{1}\right]\right)\left(V_{1}-V_{2}\right)\right\} d \chi \\
& \geqslant C^{(2)}\left(\delta_{S}\right)(1-q)\left\|\eta_{1}-\eta_{2}\right\|_{L^{2}(J)}^{2}
\end{aligned}
$$


при всех $\eta_{j} \in C(\bar{J}), \quad 0<\eta_{j} \leqslant \delta_{S}^{-1} u V_{j}=V_{0}+\int_{0}^{\chi} \eta_{j}(\xi) d \xi c j=1,2 ;$ более того, это неравенство является строгим при условии, что $\eta_{1}(M)=\eta_{2}(M)$, но $\eta_{1} \not \equiv \eta_{2}$.

Как следствие при выполнении условий (123) и (124) с q=1 лагранжева стационарная задача имеет не более одного решения $\eta_{S} \in C(\bar{J})$ такого, что $\eta_{S} \leqslant \delta_{S}^{-1}$.

ДокАЗАТЕЛЬСТво. В условиях п. 2) получим

$$
\widetilde{\mathscr{B}}\left(\eta_{1}, \eta_{2}\right) \geqslant C^{(2)}\left(\delta_{S}\right)\left\|\eta_{1}-\eta_{2}\right\|_{J}^{2}-\|\varphi\|_{L^{1}(J)}\left\|V_{1}-V_{2}\right\|_{C(\bar{J})}^{2} .
$$

Поскольку $\left\|V_{1}-V_{2}\right\|_{C(\bar{J})}^{2} \leqslant\left\|\eta_{1}-\eta_{2}\right\|_{L^{1}(J)}^{2} \leqslant M\left\|\eta_{1}-\eta_{2}\right\|_{J}^{2}$, то справедливо неравенство (125); более того, последнее неравенство для $\eta_{1}-\eta_{2}$ является строгим при условии, что $\left(\eta_{1}-\eta_{2}\right)(M)=0$, но $\eta_{1}-\eta_{2} \not \equiv 0$.

Задаче (120), (121) отвечает интегральное тождество

$$
\int_{J}\left\{\left(p_{\Gamma, S}-\widehat{p}\left(\eta_{S}\right)\right) D \varphi-\check{h}\left[V_{S}\right] \varphi\right\} d \chi=0 \quad \forall \varphi \in H^{1}(J), \quad \varphi(0)=0 .
$$

Следовательно, для любых двух решений $\eta_{S}$ и $\widetilde{\eta}_{S}$ этой задачи верно равенство $\widetilde{\mathscr{B}}\left(\widetilde{\eta}_{S}, \eta_{S}\right)=0$. Но если $\eta_{S} \not \equiv \widetilde{\eta}_{S}$, то $\widetilde{\mathscr{B}}\left(\widetilde{\eta}_{S}, \eta_{S}\right)>0$ как при $h(\nu, \chi)$, не возрастающей по $\nu$, так и в условиях п. 2) в силу (125) при условии, что $\eta_{S} \leqslant \delta_{S}^{-1}$ и $\widetilde{\eta}_{S} \leqslant \delta_{S}^{-1}$. Поэтому $\eta_{S} \equiv \widetilde{\eta}_{S}$.

Пункт 1) обобщает соответствующие утверждения в [17], [24]. В случае $f_{S}=f_{G}$, $k=2$ условия (123) и (124) сводятся к

$$
\frac{4 G M^{2}\left(M_{0}+0.5 M\right)}{r_{0}^{7} C^{(2)}\left(\rho_{\Gamma, S}\right)} \leqslant q .
$$

Применив равенство $\eta_{S}=D V_{S}$, можно исключить функцию $\eta_{S}$ из стационарной задачи (120), (121) и получить эквивалентную краевую задачу для нелинейного ОДУ второго порядка:

$$
\begin{gathered}
D \widehat{p}\left(D V_{S}\right)=\check{h}\left[V_{S}\right] \text { на } J, \\
V_{S}(0)=V_{0},\left.\quad \widehat{p}\left(D V_{S}\right)\right|_{\chi=M}=p_{\Gamma, S},
\end{gathered}
$$

относительно функции $V_{S} \in C^{1}(\bar{J})$ такой, что $\left(D V_{S}\right)_{\min }>0$.

Следуя методу малых возмущений, можно заменить $V_{S}$ на $V=V_{S}+W$ с мальм $W$ и линеаризовать оператор последней задачи:

$$
\begin{gathered}
D \widehat{p}(D V)-\check{h}[V]=D\left(\widehat{p}^{\prime}\left(D V_{S}\right) D W\right)-\breve{h}_{\nu}\left[V_{S}\right] W+\cdots \quad \text { на } J, \\
V(0)=V_{0}+W(0),\left.\quad \widehat{p}(D V)\right|_{\chi=M}-p_{\Gamma, S}=\left.\left(\widehat{p}^{\prime}\left(V_{S}\right) D W\right)\right|_{\chi=M}+\cdots,
\end{gathered}
$$

с точностью до членов второго порядка малости по отношению к $W$. Введем ассоциированную задачу на собственные значения для линеаризованного оператора:

$$
\begin{gathered}
-D\left(-\widehat{p}^{\prime}\left(\eta_{S}\right) D W\right)-\breve{h}_{\nu}\left[V_{S}\right] W=\lambda \widetilde{a}_{0} W \text { на } J \\
W(0)=0,\left.\quad\left(\widehat{p}^{\prime}\left(\eta_{S}\right) D W\right)\right|_{\chi=M}=0
\end{gathered}
$$


и назовем стационарное решение статически устойчивым, если

$$
\bar{\lambda}_{\min }\left\{\bar{\rho}_{S}\right\}>0,
$$

где $\bar{\lambda}_{\min }\left\{\bar{\rho}_{S}\right\}$ - минимальное собственное число этой самосопряженной задачи на собственные значения для ОДУ второго порядка. Конечно, здесь функции $\bar{\rho}_{S}, \eta_{S}$ и $V_{S}$ связаны последними равенствами (118) и (120); кроме того, $\widetilde{a}_{0} \in C(\bar{J})-$ некоторая функция такая, что $\left(\widetilde{a}_{0}\right)_{\min }>0$. В связи с работами [17], [24] отметим, что условие (131) очевидным образом выполнено в частном случае, когда $\breve{h}_{\nu} \leqslant 0$.

Чтобы прояснить вариационный смысл этого определения, напомним выражение для статической потенциальной энергии, отвечающей задаче (118), (119):

$$
\overline{\mathscr{F}}_{S}\left[\bar{\rho}_{S}\right]:=\int_{J}\left(\widehat{P}_{0}\left(\eta_{S}\right)+p_{\Gamma, S} \eta_{S}-\check{F}\left[r_{S}\right]\right) d \chi=\mathscr{F}_{S}\left\{\rho_{S}, R_{S}\right\},
$$

где

$$
\widehat{P}_{0}(s):=P_{0}\left(\frac{1}{s}\right)=-\int_{1}^{s} \widehat{p}(\zeta) d \zeta, \quad \check{F}\left[r_{S}\right](\chi):=F\left(r_{S}(\chi), \chi\right) .
$$

Положим также $\check{H}\left[V_{S}\right](\chi):=H\left(V_{S}(\chi), \chi\right)($ см. $(55))$ и перепишем потенциальную энергию в фороре

$$
\overline{\mathscr{F}}_{S}\left[\bar{\rho}_{S}\right]=\mathscr{P}\left[V_{S}\right]:=\int_{J}\left(\widehat{P}_{0}\left(D V_{S}\right)+p_{\Gamma, S} D V_{S}-\check{H}\left[V_{S}\right]\right) d \chi
$$

Определим подпространство $\widetilde{C}^{1}(\bar{J}):=\left\{W \in C^{1}(\bar{J}): W(0)=0\right\}$ и открытое множество $\mathscr{S}_{0}:=\left\{V \in C^{1}(\bar{J}): V(0)=V_{0},(D V)_{\min }>0\right\}$ на гиперплоскости в $C^{1}(\bar{J})$ и рассмотрим $\mathscr{P}[V]$ на $\mathscr{S}_{0}$. Для $V \in \mathscr{S}_{0}$ и $W \in \widetilde{C}^{1}(\bar{J})$ первая и вторая вариация $\mathscr{P}$ даются формулами

$$
\begin{aligned}
\delta \mathscr{P}[V](W):=\left.\frac{d}{d \tau} \mathscr{P}[V+\tau W]\right|_{\tau=0} & =\int_{J}\left\{\left(p_{\Gamma, S}-\widehat{p}(D V)\right) D W-\breve{h}[V] W\right\} d \chi, \\
\delta^{2} \mathscr{P}[V](W):=\left.\frac{d^{2}}{d \tau^{2}} \mathscr{P}[V+\tau W]\right|_{\tau=0} & =\int_{J}\left\{-\widehat{p}^{\prime}(D V)(D W)^{2}-\check{h}_{\nu}[V] W^{2}\right\} d \chi,
\end{aligned}
$$

в последней предполагается выполненным условие $h_{\nu} \in L^{1}\left(J ; C\left[V_{0}, V(M)+\varepsilon\right]\right)$ с некоторым $\varepsilon>0$. Стандартным образом стационарная задача в форме (127), (128) эквивалентна соотношению

$$
\delta \mathscr{P}\left[V_{S}\right](W)=0 \quad \forall W \in \widetilde{C}^{1}(\bar{J}),
$$

cр. с (126), т.е. решения $V_{S}$ задачи $(127),(128)$, и только они, являются стационарньми точками $\mathscr{P}$. Более того, следующее условие (ср. с [4])

$$
\overline{\mathscr{J}}[W]:=\delta^{2} \mathscr{P}\left[V_{S}\right](W)>0 \quad \forall W \in \widetilde{C}^{1}(\bar{J}), \quad W \not \equiv 0,
$$

эквивалентно условию статической устойчивости $(131)$, и $\overline{\mathscr{J}}[W]$ является энергетическим функционалом, отвечающим за даче на собственные значения (129), (130).

Установим непосредственную связь с соответствующими понятиями из $\S 2$ и, в частности, эквивалентность двух определений статической устойчивости (35) и (131). 
УТВЕРЖДЕНИЕ 6 . Пусть справедливъц свойства $\left(f_{S}\right)_{\chi} \in L^{1}\left(\left(r_{0}, R_{S}\right) ; C(\bar{J})\right)$ $u\left(f_{S}\right)_{r},\left(f_{S}\right)_{\chi} \in L^{1}\left(J ; C\left[r_{0}, R_{S}\right]\right)$. Тогда

$$
\mathscr{J}_{R_{S}}[w]=\bar{J}\left[\eta_{S} \bar{w}\right] \quad \forall w \in \widetilde{H}^{1}\left(\Omega_{S}\right),
$$

əде $\bar{w}(\chi)=w\left(r_{S}(\chi)\right)$ на J. Как следствие

$$
\lambda_{\min }\left\{\rho_{S}, R_{S}\right\}=\bar{\lambda}_{\min }\left\{\bar{\rho}_{S}\right\}
$$

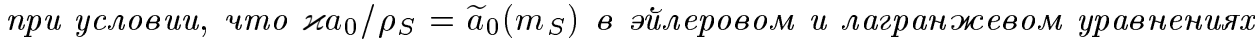
на собственные значения (33) и (129).

ДокАЗАТЕЛЬСТво. После замены переменной $\chi=m_{S}(r)$, используя функцию $h$ вместо $f_{S}$, получаем

$$
\mathscr{J}_{R_{S}}[w]=\int_{J}\left\{p^{\prime}\left(\bar{\rho}_{S}\right)(D \bar{w})^{2}+\frac{\check{h}_{\chi}\left[V_{S}\right]}{\bar{\rho}_{S}} \bar{w}^{2}\right\} d \chi-\left.\left(\frac{\check{h}\left[V_{S}\right]}{\bar{\rho}_{S}} \bar{w}^{2}\right)\right|_{\chi=M},
$$

где $w(r)=\bar{w}\left(m_{S}(r)\right)$ на $\bar{\Omega}_{S}$. Положим $W:=\eta_{S} \bar{w}$ и выполним преобразования

$$
\begin{aligned}
p^{\prime}\left(\bar{\rho}_{S}\right)(D \bar{w})^{2} & =\bar{\rho}_{S}^{2} p^{\prime}\left(\bar{\rho}_{S}\right)(D W)^{2}+\bar{\rho}_{S}\left(D p\left(\bar{\rho}_{S}\right)\right) D\left(W^{2}\right)+\left(D \bar{\rho}_{S}\right)\left(D p\left(\bar{\rho}_{S}\right)\right)(D W)^{2} \\
& =-\widehat{p}^{\prime}\left(\eta_{S}\right)(D W)^{2}+D\left(\bar{\rho}_{S} \check{h}\left[V_{S}\right] W^{2}\right)-\bar{\rho}_{S}\left(D \breve{h}\left[V_{S}\right]\right) W^{2}
\end{aligned}
$$

применив уравнение $D p\left(\bar{\rho}_{S}\right)=\check{h}\left[V_{S}\right]$. Поэтому

$$
\mathscr{J}_{R_{S}}[w]=\int_{J}\left\{-\widehat{p}^{\prime}\left(\eta_{S}\right)(D W)^{2}-\bar{\rho}_{S}\left(D \check{h}\left[V_{S}\right]-\check{h}_{\chi}\left[V_{S}\right]\right) W^{2}\right\} d \chi=\bar{J}[W] .
$$

Поскольку

$$
\int_{\Omega_{S}} a_{0} w^{2} d r=\int_{J} \frac{\bar{\rho}_{S}}{r_{S}^{k}} \bar{a}_{0} W^{2} d \chi
$$

с $a_{0}=\bar{a}_{0}\left(m_{S}\right)$, то доказательство завершено.

Покажем, что статически устойчивые решения изолированы в сильном смысле.

УТВЕРЖДЕНИЕ 7. Предположим, что $p^{\prime}>0 u$

$$
\begin{gathered}
\left\|\left(f_{S}\right)_{r}\right\|_{L^{1}\left(J ; C\left[r_{0}, R_{S}+\varepsilon_{S}\right]\right)} \leqslant N, \\
\|\widetilde{\theta}(\cdot)[\delta]\|_{L^{1}(J)} \rightarrow 0 \quad n p u \quad \delta \rightarrow 0^{+}
\end{gathered}
$$

$\partial \Omega Я$

$$
\widetilde{\theta}(\chi)[\delta]:=\sup _{0<\Delta \leqslant \delta}\left\|\left(f_{S}\right)_{r}(r+\Delta, \chi)-\left(f_{S}\right)_{r}(r, \chi)\right\|_{C\left[r_{0}, R_{S}+\varepsilon_{S}-\Delta\right]}
$$

с некоторым $\varepsilon_{S}>0$. Если стационарное решение $\bar{\rho}_{S}$ статически устойчиво, то при достаточно малом $\varepsilon_{0}>0$ не существует другого решения $\bar{\rho}_{S}^{(1)}$ такого, что $\left|R_{S}-R_{S}^{(1)}\right|<\varepsilon_{0}$, где $R_{S}$ и $R_{S}^{(1)}$ - соответствующие радиусъь свободных границ. 
ДоказАТЕЛЬство. Рассуждения аналогичны примененньм в утверждении 4 и даже проще (поскольку теперь область $J$ фиксирована). Они базируются на изучении вспомогательной задачи Коши

$$
\begin{gathered}
D p_{S}=\check{h}\left[V_{S}\right], \quad D V_{S}=\widehat{p}^{-1}\left(p_{S}\right) \quad \text { на } J \\
p_{S}(M)=p_{\Gamma, S}, \quad V_{S}(M)=\nu
\end{gathered}
$$

для функций $p_{S}=\widehat{p}\left(\eta_{S}\right)$ и $V_{S}$ с заданным $\nu>V_{0}$ и выводе соотношений

$$
\int_{J}\left\{-\widehat{p}^{\prime}\left(D V_{S}+\varepsilon W\right)(D W)^{2}-\breve{h}_{\nu}\left[V_{S}+\varepsilon W\right] W^{2}\right\} d \chi=(1+o(\varepsilon)) \overline{\mathscr{J}}[W] \geqslant \delta_{0}\|W\|_{H^{1}(J)}^{2}
$$

при любых $W \in \widetilde{C}^{1}(\bar{J}),\|W\|_{C^{1}(\bar{J})}=1$ и с $0<\varepsilon \leqslant \varepsilon_{0}$ и $\delta_{0}>0$.

Отметим, что в силу второго соотношения (120) условие $\left|R_{S}-R_{S}^{(1)}\right|<\varepsilon_{0}$ может быть эквивалентным образом заменено на

$$
\left|\int_{J}\left(\eta_{S}-\eta_{S}^{(1)}\right) d \chi\right|<\varepsilon_{1}
$$

с $\eta_{S}^{(1)}:=1 / \bar{\rho}_{S}^{(1)}$ и достаточно малым $\varepsilon_{1}>0$.

Назовем $V \in \mathscr{S}_{0}$ точкой локального квадратичного минимума Я્Р, если

$$
\mathscr{P}[V+W]-\mathscr{P}[V] \geqslant \delta_{0}\|W\|_{H^{1}(J)}^{2} \quad \forall W \in \widetilde{C}^{1}(\bar{J}), \quad\|W\|_{C^{1}(\bar{J})} \leqslant \varepsilon_{0},
$$

при некоторых $\varepsilon_{0}>0$ и $\delta_{0}>0$.

УТВЕРЖДЕНИЕ 8. Предположим, что выполнены условие $p^{\prime}>0$ вместе $c(134) u$ (135). Функиия $V \in \mathscr{S}_{0}$ является точкой локального квадратичного минимума Я тогда и только тогда, когда $V_{S}=V$ является решением задачи (127), (128), удовлетворяющим условию статической устойчивости (133).

ДоказАтЕльство. Пусть $V \in \mathscr{S}_{0}$ и $\|W\|_{C^{1}(\bar{J})}=1$. По формуле Тейлора

$$
\mathscr{P}[V+\varepsilon W]=\mathscr{P}[V]+\delta \mathscr{P}[V](\varepsilon W)+\left.\frac{1}{2} \frac{d^{2}}{d \tau^{2}} \mathscr{P}[V+\tau \varepsilon W]\right|_{\tau=\widetilde{\tau}}
$$

при всех $0<\varepsilon<\left[(D V)_{\min }\right]^{-1}$ и некотором $\widetilde{\tau} \in[0,1]$. Поскольку аналогично равенству в (138)

$$
\begin{aligned}
\left.\frac{d^{2}}{d \tau^{2}} \mathscr{P}[V+\tau \varepsilon D W]\right|_{\tau=\widetilde{\tau}} & =\varepsilon^{2} \int_{J}\left\{-\widehat{p}^{\prime}(D V+\widetilde{\tau} \varepsilon W)(D W)^{2}-\check{h}_{\nu}[V+\widetilde{\tau} \varepsilon W] W^{2}\right\} d \chi \\
& =(1+o(\varepsilon)) \delta^{2} \mathscr{P}[V](\varepsilon W),
\end{aligned}
$$

то условие (139) эквивалентно (132) вместе с

$$
(1+o(\varepsilon)) \overline{\mathscr{J}}[W] \geqslant 2 \delta_{0}\|W\|_{H^{1}(J)}^{2} \quad \forall W \in \widetilde{C}^{1}(\bar{J}), \quad 0<\varepsilon \leqslant \varepsilon_{0},
$$

для $V_{S}=V$ (в последнем неравенстве условие $\|W\|_{C^{1}(\bar{J})}=1$ пропадает). 
Замечание 7. Свойства (12), (13) влекут оценку снизу

$$
\mathscr{P}[V] \geqslant(2 N)^{-1} V(M)-K
$$

и, в частности, $\mathscr{P}[V] \rightarrow+\infty$ при условии, что $\|V\|_{C(\bar{J})}=V(M) \rightarrow \infty$, для $V \in \mathscr{S}_{0}$. Действительно,

$$
\begin{aligned}
\widehat{P}_{0}(\zeta) & =\Pi\left(\zeta, \zeta_{0}\right)-\left(\zeta-\zeta_{0}\right) \widehat{p}\left(\zeta_{0}\right)+\widehat{P}_{0}\left(\zeta_{0}\right) \\
\text { c } \Pi\left(\zeta, \zeta_{0}\right) & :=\int_{\zeta_{0}}^{\zeta}\left(\widehat{p}\left(\zeta_{0}\right)-\widehat{p}(\xi)\right) d \xi \geqslant 0 \quad \forall \zeta>0, \quad \zeta_{0}>0 ;
\end{aligned}
$$

как следствие имеем соотношения при всех $\zeta_{0}>0, \varepsilon>0$

$$
\begin{aligned}
\mathscr{P}[V] & \geqslant \int_{J}\left\{\left(p_{\Gamma, S}-\widehat{p}\left(\zeta_{0}\right)\right) D V-\left(f_{0}+\varepsilon\right) V-C_{1 \varepsilon}\right\} d \chi-C_{0}\left(\zeta_{0}\right) \\
& =\left\{p_{\Gamma, S}-\widehat{p}\left(\zeta_{0}\right)-M\left(f_{0}+\varepsilon\right)\right\} V(M)-C
\end{aligned}
$$

с $C=C\left(\varepsilon, \zeta_{0}, p_{\Gamma, S}, V_{0}, M\right)$, которые и влекут желаемую оценку при достаточно малых $\zeta_{0}^{-1}$ и $\varepsilon$.

ЗАМЕчАниЕ 8 . Функция $\widehat{P}_{0}$ строго выпукла при вьполнении первого и второго условий $(10)$, а функция $-H(\nu, \chi)$ выпукла по $\nu$, если $h(\nu, \chi)$ не возрастает по $\nu$ при всех $\chi \in \bar{J}$ (в частности, если $h_{\nu} \leqslant 0$ ). Следовательно, при выполнении этих условий и (11) функционал $\mathscr{P}$ является строго выпуклым на $\mathscr{S}_{0}$ и поэтому он имеет не более одной стационарной точки (фактически точки минимума), cр. с п. 1) в утверждении 5 . Более того, при выполнении условий $(10),(11),(14)$ вместе с $p^{\prime}>0$ и $h_{\nu} \leqslant 0$ получим

$$
\delta^{2} \mathscr{P}[V](W) \geqslant \int_{J}-\hat{p}^{\prime}(D V)(D W)^{2} d \chi \geqslant c_{0}(V) \int_{J}(D W)^{2} d \chi,
$$

где $c_{0}(V):=\min _{\left[\zeta_{0}, \zeta_{1}\right]}\left|\hat{p}^{\prime}(\zeta)\right|>0$ с $0<\zeta_{0} \leqslant D V \leqslant \zeta_{1}$, и тем самым выполнено условие (133).

Обсудим неравенство (125). В силу равенства

$$
\widetilde{\mathscr{B}}\left(\eta_{1}, \eta_{2}\right)=\left(\delta \mathscr{P}\left[V_{1}\right]-\delta \mathscr{P}\left[V_{2}\right]\right)\left(V_{1}-V_{2}\right)
$$

неравенство (125) с некоторым $0 \leqslant q<1$ обеспечивает строгую монотонность $\delta \mathscr{P}$ на подмножестве $\left\{V \in \mathscr{S}_{0}: D V \leqslant \delta_{S}^{-1}\right\}$. Введем также связанное с этим условие на стационарное решение $\eta_{S}$, которое будет применено в следуюшем параграфе: для всех достаточно больших $N_{0}$

$$
\widetilde{\mathscr{B}}\left(\eta_{1}, \eta_{S}\right) \geqslant \delta_{0}\left(N_{0}\right)\left\|\eta_{1}-\eta_{S}\right\|_{J}^{2} \text { при любых } \eta_{1} \in C(\bar{J}), \quad N_{0}^{-1} \leqslant \eta_{1} \leqslant N_{0},
$$

с некоторьм $\delta_{0}\left(N_{0}\right)>0$. Конечно, при выполнении этого условия других стационарных решений нет; кроме того, использовав формулу Ньютона-Лейбница для функционалов и свойство стационарности (132), получим

$$
\begin{aligned}
\mathscr{P}\left[V_{1}\right]-\mathscr{P}\left[V_{S}\right] & =\int_{0}^{1}\left(\delta \mathscr{P}\left[\alpha V_{1}+(1-\alpha) V_{S}\right]-\delta \mathscr{P}\left[V_{S}\right]\right)\left(V_{1}-V_{S}\right) d \alpha \\
& =\int_{0}^{1} \widetilde{\mathscr{B}}\left(\alpha \eta_{1}+(1-\alpha) \eta_{S}, \eta_{S}\right) \frac{d \alpha}{\alpha} \\
& \geqslant \frac{\delta_{0}\left(N_{0}\right)}{2}\left\|\eta_{1}-\eta_{S}\right\|_{J}^{2}=\frac{\delta_{0}\left(N_{0}\right)}{2}\left\|D\left(V_{1}-V_{S}\right)\right\|_{J}^{2}
\end{aligned}
$$


при достаточно больших $N_{0}$ (таких, что $N_{0}^{-1} \leqslant \eta_{S} \leqslant N_{0}$ ). Таким образом, $V_{S}$ является точкой глобального квадратичного минимума $\mathscr{P}$ на подмножествах $\left\{V \in \mathscr{S}_{0}: N_{0}^{-1} \leqslant D V \leqslant N_{0}\right\}$, а условие (141) обеспечивает строгую монотонность $\delta \mathscr{P}\left[V_{1}\right]$ по отношению к фиксированному $V_{1}=V_{S}$ на этих подмножествах.

В заключение параграффа представим два результата: о статической устойчивости и о единственности стационарных решений без предположения $h_{\nu} \leqslant 0$, которые важны, в частности, в астрофизическом контексте. Определим так называемый первый адиабатический показатель

$$
\Gamma_{1}(s):=\frac{s p^{\prime}(s)}{p(s)}=-\left.\frac{\widehat{p}^{\prime}(\zeta)}{\zeta \widehat{p}(\zeta)}\right|_{\zeta=1 / s} \quad \text { при } s>0 .
$$

УТВеРЖДЕНИЕ 9. Предположим, что $p^{\prime}>0,\left(f_{S}\right)_{r} \in C\left(\overline{\mathbb{R}}^{+} \times[0, M]\right) u$ функция $h^{(0)}$ удовлетворяет равенству $h_{\nu}=h h^{(0)}$ (ясно, что $h^{(0)}=h_{\nu} / h н а$ множестве, где $h \neq 0$ ) и условиям

$$
\begin{array}{ll}
h^{(0)}, h_{\nu}^{(0)}, h_{\chi}^{(0)} \in L^{1}\left(J ; C\left[V_{0}, \nu\right]\right) & \forall \nu>V_{0}, \\
\frac{\left(h^{(0)}(\nu, \chi)\right)^{2}}{\Gamma_{1}(s)}-h_{\nu}^{(0)}(\nu, \chi)-s h_{\chi}^{(0)}(\nu, \chi) \leqslant 0 & \forall s>0, \nu>V_{0}, \chi \in J, \\
h^{(0)}(\nu, M) \leqslant 0 & \forall \nu>V_{0} .
\end{array}
$$

Тогда условие (133) выполняется для любого стационарного решения.

ДоказАтельство. Ясно, что $h^{(0)} \in C\left(\left[V_{0}, \infty\right) \times[0, M]\right)$ благодаря (144). Применив соотношения $h_{\nu}=h^{(0)} h$ и (120) и проинтегрировав по частям, при любых $W \in \widetilde{C}^{1}(\bar{J})$ можно преобразовать второе слагаемое в $\mathscr{J}[W]$ следуюшим образом:

$$
\begin{aligned}
-\int_{J} \check{h}_{\nu}\left[V_{S}\right] W^{2} d \chi=-\int_{J} h^{(0)}\left[V_{S}\right]\left(D \widehat{p}\left(\eta_{S}\right)\right) W^{2} d \chi \\
=\int_{J}\left\{2 h^{(0)}\left[V_{S}\right] \widehat{p}\left(\eta_{S}\right)(D W) W+\left(h_{\nu}^{(0)}\left[V_{S}\right] \eta_{S}+h_{\chi}^{(0)}\left[V_{S}\right]\right) \widehat{p}\left(\eta_{S}\right) W^{2}\right\} d \chi \\
\quad-h^{(0)}\left[V_{S}\right](M) p_{\Gamma, S} W^{2}(M) .
\end{aligned}
$$

В силу условия (145) и формулы (143)

$$
\left|\int_{J} 2 h^{(0)}\left[V_{S}\right] \widehat{p}\left(\eta_{S}\right)(D W) W d \chi\right| \leqslant \int_{J}-2 \widehat{p}^{\prime}\left(\eta_{S}\right) \sqrt{a}|D W||W| d \chi
$$

с $a:=-\left(h_{\nu}^{(0)}\left[V_{S}\right] \eta_{S}+h_{\chi}^{(0)}\left[V_{S}\right]\right) / \widehat{p}^{\prime}\left(\eta_{S}\right)$. Воспользовавшись также условиями (146) и $p^{\prime}>0$, получим

$$
\mathscr{J}[W] \geqslant \int_{J}-\widehat{p}^{\prime}\left(\eta_{S}\right)(|D W|-\sqrt{a}|W|)^{2} d \chi \geqslant c_{0} \int_{J}(|D W|-\sqrt{a}|W|)^{2} d \chi \geqslant 0
$$

с $c_{0}>0$ (см. (140)). Последний интеграл равен нулю, если и только если $|D W|=$ $\sqrt{a}|W|$ на $\bar{J}$; следовательно, $D W=\sigma_{0} \sqrt{a} W$ с функцией $\sigma_{0}(\chi):=1$ в случаях $D W(\chi) \geqslant 0, W(\chi) \geqslant 0$ или $D W(\chi)<0, W(\chi)<0$ и $\sigma_{0}(\chi):=-1$ в остальных случаях, которая измерима на $J$. Поскольку $W(0)=0$, то отсюда $W \equiv 0$.

Отметим, что если $1 / \Gamma_{1}(s)=O(1)$ при $s \rightarrow \infty$, то условие (145) влечет, что $h_{\chi}^{(0)} \geqslant 0$. Далее, если условия (145) и (146) выполнены только для $s=\bar{\rho}_{S}(\chi)$ и $w=V_{S}(\chi)$ на $J$ для частного стационарного решения, условие (133) выполнено для этого решения. 
УТВеРЖДЕНИЕ 10. Предположим, ито $p^{\prime}>0,\left(f_{S}\right)_{r} \in C\left(\overline{\mathbb{R}}^{+} \times\left[0, M_{1}\right]\right) u$ функция $h^{(1)}$ удовлетворяет равенству $h=h_{\nu} h^{(1)}$ (ясно, что $h^{(1)}=h / h_{\nu}$ на множестве, где $h_{\nu} \neq 0$ ) и условиям

$$
\begin{aligned}
h^{(1)}, h_{\nu}^{(1)}, h_{\chi}^{(1)} \in L^{1}\left(0, M_{1} ; C\left[V_{0}, \nu\right]\right) & \forall \nu>V_{0}, \\
\frac{1}{\Gamma_{1}(s)}+h_{\nu}^{(1)}(\nu, \chi)+s h_{\chi}^{(1)}(\nu, \chi) \leqslant 0 & \forall s>0, \nu>V_{0}, \chi \in\left(0, M_{1}\right), \\
\lim _{(\nu, \chi) \rightarrow\left(V_{0}^{+}, 0^{+}\right)} h^{(1)}(\nu, \chi) \leqslant 0 . &
\end{aligned}
$$

Тогда стационарная задача (118), (119) имеет не более одного решения.

ДоКАЗАТЕЛЬСТво. Будем использовать метод стрельбы и введем задачу Коши

$$
\begin{gathered}
D p_{S}=\check{h}\left[V_{S}\right], \quad D V_{S}=\widehat{p}^{-1}\left(p_{S}\right) \quad \text { на }\left(0, M_{1}\right), \\
\left.p_{S}\right|_{\chi=0}=\lambda,\left.\quad V_{S}\right|_{\chi=0}=V_{0},
\end{gathered}
$$

с искомыми функциями $p_{S}(\chi, \lambda), V_{S}(\chi, \lambda)($ ср. с (136), (137)). При любом значении параметра стрельбы $\lambda>0$ сушествует единственное решение этой задачи, удовлетворяющее неравенству $p_{S}(\chi, \lambda)>0$ фактически при $\chi \in[0, \bar{M}(\lambda))$, где либо $\left.p_{S}\right|_{\chi=\bar{M}(\lambda)}=0$ и $0<\bar{M}(\lambda)<M_{1}$, либо $\bar{M}(\lambda)=M_{1}$.

Ясно, что для любого решения $\eta_{S}$ задачи $(120),(121)$ функции $p_{S}:=\widehat{p}\left(\eta_{S}\right)$ и $V_{S}$ удовлетворяют (150), (151) при некотором $\lambda>0$. Покажем, что это возможно только при единственном значении $\lambda$.

С этой целью введем также производные по параметру

$$
\dot{p}_{S}=\frac{\partial}{\partial \lambda} p_{S}, \quad \dot{V}_{S}=\frac{\partial}{\partial \lambda} V_{S},
$$

которые корректно определены и удовлетворяют линейной задаче Коши

$$
\begin{gathered}
D \dot{p}_{S}=\check{h}_{\nu}\left[V_{S}\right] \dot{V}_{S}, \quad D \dot{V}_{S}=\left(\widehat{p}^{-1}\right)^{\prime}\left(p_{S}\right) \dot{p}_{S} \quad \text { на }[0, \bar{M}(\lambda)), \\
\left.\dot{p}_{S}\right|_{\chi=0}=1,\left.\quad \dot{V}_{S}\right|_{\chi=0}=0
\end{gathered}
$$

(см., например, [31; раздел V.3]). В силу $(153)$ и $\left(\widehat{p}^{-1}\right)^{\prime}<0$ справедливы неравенства

$$
\dot{p}_{S}(\chi, \lambda)>0, \quad D \dot{V}_{S}(\chi, \lambda)<0, \quad \dot{V}_{S}(\chi, \lambda)<0
$$

на некотором полусегменте $[0, a(\lambda))$, где либо $\left.\dot{p}_{S}\right|_{\chi=a(\lambda)}=0$ и $0<a(\lambda)<\bar{M}(\lambda)$, либо $a(\lambda)=\bar{M}(\lambda)$. Докажем, что реализуется только вторая возможность.

Рассмотрим вспомогательную функцию $\psi:=p_{S} D \dot{V}_{S}-\dot{p}_{S} D\left(h^{(1)}\left[V_{S}\right]\right)$. В силу вторых уравнений (150) и (152) найдем, что

$$
\begin{aligned}
\psi & =p_{S}\left(\widehat{p}^{-1}\right)^{\prime}\left(p_{S}\right) \dot{p}_{S}-\dot{p}_{S}\left(h_{\nu}^{(1)}\left[V_{S}\right] \widehat{p}^{-1}\left(p_{S}\right)+h_{\chi}^{(1)}\left[V_{S}\right]\right) \\
& =-\dot{p}_{S} \widehat{p}^{-1}\left(p_{S}\right)\left\{\frac{1}{\Gamma_{1}\left(p^{-1}\left(p_{S}\right)\right)}+h_{\nu}^{(1)}\left[V_{S}\right]+p^{-1}\left(p_{S}\right) h_{\chi}^{(1)}\left[V_{S}\right]\right\} .
\end{aligned}
$$

Поэтому $\psi \geqslant 0$ на $(0, a(\lambda))$ согласно условию (148). 
С другой стороны, предположим, что $a(\lambda)<\bar{M}(\lambda)$ при некотором $\lambda$. В силу первых уравнений $(150)$ и (152) найдем, что $-\left(D p_{S}\right) \dot{V}_{S}+\left(D \dot{p}_{S}\right) h^{(1)}\left[V_{S}\right]=0$ и, следовательно,

$$
\int_{0}^{a(\lambda)} \psi d \chi=\left.\left(p_{S} \dot{V}_{S}-\dot{p}_{S} h^{(1)}\left[V_{S}\right]\right)\right|_{0} ^{a(\lambda)}
$$

где $\left.h^{(1)}\left[V_{S}\right]\right|_{\chi=0}:=\lim _{\chi \rightarrow 0^{+}} h^{(1)}\left[V_{S}\right] \leqslant 0$ по условию (149). В силу соотношений $\left.p_{S}\right|_{\chi=a(\lambda)}>0,\left.\dot{p}_{S}\right|_{\chi=a(\lambda)}=0$ и $\left.\dot{V}_{S}\right|_{\chi=a(\lambda)}<0$ и начальных условий $(153)$ выведем

$$
\int_{0}^{a(\lambda)} \psi d \chi=\left.\left(p_{S} \dot{V}_{S}\right)\right|_{\chi=a(\lambda)}+\left.h^{(1)}\left[V_{S}\right]\right|_{\chi=0} \leqslant\left.\left. p_{S}\right|_{\chi=a(\lambda)} \dot{V}_{S}\right|_{\chi=a(\lambda)}<0
$$

что приводит к противоречию.

Наконец, поскольку множество $\{(\chi, \lambda): 0<\chi<\bar{M}(\lambda), \lambda>0\}$ открытое (см. [31]) и как $p_{S}>0$, так и $\dot{p}_{S}>0$ на нем, то функция $\bar{M}$ не убывает на $\mathbb{R}^{+}$, и при любом фиксированном $0 \leqslant \chi<\sup _{\lambda>0} \bar{M}(\lambda)$ функция $p_{S}(\chi, \lambda)$ определена на полупрямой $\lambda>\lambda_{0}(\chi)$ и возрастает на ней. Следовательно, функция $p_{S}$ может удовлетворять условию $p_{S}(M, \lambda)=p_{\Gamma, S}$ для не более чем одного значения $\lambda$.

ЗАмЕчАниЕ 9. Если $h^{(0)}$ не вырождается (в частности, если $h$ не вырождается), то можно положить $h^{(1)}:=1 / h^{(0)}$, и условия $(144),(145)$ влекут $(147),(148)$ с $M$ в роли $M_{1}$. Наоборот, если $h^{(1)}$ не вырождается (в частности, если $h_{\nu}$ не вырождается), то можно положить $h^{(0)}:=1 / h^{(1)}$, и условия $(147),(148)$ влекут (144), (145).

СлЕДСТВИЕ 3. В случае $f_{S}=f_{G}, k=2 u \Gamma_{1} \geqslant 4 / 3$ стационарная задача имеет единственное решение, более того, оно статически устойчиво.

ДокАЗАТЕльство. В этом случае $h(\nu, \chi)=-G\left(M_{0}+\chi\right) /(3 \nu)^{4 / 3}$ и $h^{(1)}(\nu, \chi)=$ $1 / h^{(0)}(\nu, \chi)=-3 \nu / 4$, поэтому условия следствия 1 выполнены, а условия утверждений 9 и 10 сводятся к $\Gamma_{1} \geqslant 4 / 3$. Обратим внимание на вырождение $\left.h\right|_{\chi=0}=$ $\left.\check{h}_{\nu}\right|_{\chi=0}=0$ в случае $M_{0}=0$.

Отметим, что условие $\Gamma_{1} \geqslant \gamma_{0}$ с некоторым $\gamma_{0}>0$ эквивалентно условию $\left(s^{-\gamma_{0}} p(s)\right)^{\prime} \geqslant 0$ при $s>0$, которое влечет, что $p(s) \leqslant p(1) s^{\gamma_{0}}$ при $0<s \leqslant 1$ и $p(s) \geqslant p(1) s^{\gamma_{0}}$ при $s \geqslant 1$.

\section{§5. Оценки скорости стабилизации в лагранжевых координатах}

По ряду причин интересно дополнить эйлеровы оценки скорости стабилизации, данные в $\S 3$, аналогичными лагранжевыми.

Рассмотрев $\chi=m(r, t)$ из уравнения (4) как новую независимую переменную вместе с $t$ и приняв во внимание закон сохранения массы, задачу со свободной границей (1)-(3) можно преобразовать в задачу

$$
\begin{aligned}
D_{t} \check{\eta} & =D(\check{\varkappa} \check{v}) \quad \text { c } \check{\eta}:=\frac{1}{\check{\rho}} \\
D_{t} \check{v} & =\check{\varkappa} D[\mu(\check{\rho}) \check{\rho} D(\check{\varkappa} \check{v})-p(\check{\rho})]+\check{f}[\check{r}], \\
\check{V} & :=\frac{1}{k+1} \check{r}^{k+1}=V_{0}+\int_{0}^{\chi} \check{\eta}(\xi, t) d \xi
\end{aligned}
$$


в фиксированной области $Q_{L}:=J \times \mathbb{R}^{+}$. Связи между старыми неизвестными $\rho(r, t), v(r, t), m(r, t)$ и новыми $\check{\rho}(\chi, t), \check{v}(\chi, t), \check{r}(\chi, t)$ имеют вид:

$$
\rho(r, t)=\check{\rho}(m(r, t), t), \quad v(r, t)=\check{v}(m(r, t), t), \quad r=\check{r}(m(r, t), t) \quad \text { в } \bar{Q},
$$

конечно, $\check{\rho}>0$ и $\check{r} \geqslant r_{0}$. Кроме того, теперь $D$ и $D_{t}$ обозначают частные производные по новым переменным $\chi$ и $t$, а $\check{\varkappa}=\check{r}^{k}$ и $\breve{f}[\check{r}](\chi, t)=f(\check{r}(\chi, t), \chi, t)$. Уравнения дополняются следуюшими граничными и начальными условиями:

$$
\begin{gathered}
\left.\check{v}\right|_{\chi=0}=0,\left.\quad\left[\mu(\check{\rho}) \check{\rho} D(\check{\varkappa} \check{v})-2 k \mu_{1}(\check{\rho}) \frac{\check{v}}{\check{r}}-p(\check{\rho})\right]\right|_{\chi=M}=-p_{\Gamma}(t) \quad \text { при } t>0, \\
\left.\check{\rho}\right|_{t=0}=\check{\rho}^{0}(\chi),\left.\quad \check{v}\right|_{t=0}=\check{v}^{0}(\chi) \quad \text { при } \chi \in J
\end{gathered}
$$

где $\check{\rho}^{0}\left(m^{0}(r)\right)=\rho^{0}(r)$ и $\check{v}^{0}\left(m^{0}(r)\right)=v^{0}(r)$ на $\Omega_{0}$ с $m^{0}(r):=\int_{r_{0}}^{r}\left(\rho^{0} \varkappa\right)(q) d q$.

Применив $D_{t}$ к (157) и воспользовавшись (155) вместе с $\left.\check{v}\right|_{\chi=0}=0$, получим стандартное уравнение, связывающее $\check{r}$ и $\check{v}$ :

$$
D_{t} \check{r}=\check{v} .
$$

Стационарная задача, отвечающая задаче (155)-(159), это задача (118), (119). Введем функции

$$
A(s):=\int_{1}^{s} \frac{\mu(\zeta)}{\zeta} d \zeta, \quad \widehat{A}(s):=A\left(\frac{1}{s}\right)=-\int_{1}^{s} \mu\left(\frac{1}{\zeta}\right) \frac{d \zeta}{\zeta} .
$$

Лагранжева форма закона сохранения энергии выглядит следующим образом:

$$
\begin{gathered}
\frac{d}{d t}(\check{\mathscr{E}}+\check{\mathscr{F}}[\check{\rho}])+\int_{J} \mu(\check{\rho}) \check{\rho}(D(\check{\varkappa} \check{v}))^{2} d \chi-\left.2 k\left(\mu_{1}(\check{\rho}) \frac{\check{\varkappa} \check{v}^{2}}{\check{r}}\right)\right|_{\chi=M} \\
=\int_{J} \Delta \check{f}[\check{r}] \check{v} d \chi-\left.\Delta p_{\Gamma}(\check{\varkappa} \check{v})\right|_{\chi=M}
\end{gathered}
$$

с функционалами

$$
\check{\mathscr{E}}:=\frac{1}{2} \int_{J} \check{v}^{2} d \chi=\mathscr{E}, \quad \check{\mathscr{F}}[\check{\rho}]:=\int_{J}\left(\widehat{P}_{0}(\check{\eta})+p_{\Gamma, S} \check{\eta}-\check{F}[\check{r}]\right) d \chi=\mathscr{F}[\rho]
$$

и функциями $\check{F}[\check{r}](\chi, t)=F(\check{r}(\chi, t), \chi), \Delta \check{f}[\check{r}](\chi, t)=\Delta f(\check{r}(\chi, t), \chi, t)$.

УТВеРЖДЕНИЕ 11. 1) Пусть выполнены условия теоремы 1, n. 1) и n. 2) вместе с $N^{-1} \leqslant \rho^{0} u$

существует постоянная $\delta_{S}>0$ такая, что любое әйлерово стационарное решение $\left\{\rho_{S}, R_{S}\right\}$ удовлетворяет оченке снизу $\delta_{S} \leqslant \rho_{S}$ на $\bar{\Omega}_{S}$.

Тогда справедлива оценка снизу (70) для $\rho$ и для любой последовательности $t_{n} \rightarrow+\infty$ существует подпоследовательность $\theta_{n}$ такая, ито

$$
\check{\eta}\left(\cdot, \theta_{n}\right) \rightarrow \eta_{*}(\cdot) \quad \text { *-слабо в } L^{\infty}(J) .
$$


Более того, для любой последовательности $\theta_{n} \rightarrow \infty, \theta_{n} \geqslant 0$, удовлетворяющей этому свойству, фактически $\bar{\rho}_{S}:=1 / \eta_{*}$ служит лагранжсвым стационарным решением, удовлетворяющим равенству $\overline{\mathscr{F}}_{S}\left[\bar{\rho}_{S}\right]=\mathscr{F}^{(S)}$, и справедливо предельное соотношение

$$
\check{\rho}\left(\cdot, \theta_{n}\right) \rightarrow \bar{\rho}_{S}(\cdot) \quad \text { в } \quad L^{\lambda}(J) \quad \forall 1 \leqslant \lambda<\infty .
$$

2) Пусть дополнительно $p^{\prime}>0 н а \mathbb{R}^{+}$. Предположим, что среди стачионарных решений, удовлетворяющих (164) с $\lambda=1$ для некоторой последовательности $\theta_{n} \rightarrow \infty, \theta_{n} \geqslant 0$, и равенству $\overline{\mathscr{F}}_{S}\left[\bar{\rho}_{S}\right]=\mathscr{F}^{(S)}$, существует статически устойчивое решение, а также выполнены условия на $f_{S}$

$$
\left\|\left(f_{S}\right)_{r}\right\|_{L^{1}\left(J ; C\left[r_{0}, R_{S}+\varepsilon_{S}\right]\right)} \leqslant N, \quad\|\widetilde{\theta}(\cdot)[\delta]\|_{L^{1}(J)} \rightarrow 0 \quad n p u \quad \delta \rightarrow 0^{+}
$$

$\partial \Omega я$

$$
\widetilde{\theta}(\chi)[\delta]=\sup _{0<\Delta \leqslant \delta}\left\|\left(f_{S}\right)_{r}(r+\Delta, \chi)-\left(f_{S}\right)_{r}(r, \chi)\right\|_{C\left[r_{0}, R_{S}+\varepsilon_{S}-\Delta\right]}
$$

с некоторым $\varepsilon_{S}>0$, т.е. условия (134) и (135). Тогда это решение является единственным, удовлетворяющим (164), и поэтому

$$
\check{\rho}(\cdot, t) \rightarrow \bar{\rho}_{S}(\cdot) \quad \text { в } \quad L^{\lambda}(J) \quad \forall 1 \leqslant \lambda<\infty \quad \text { npu } t \rightarrow \infty .
$$

3) Пусть выполнены условия пn. 1) и 2) вместе с условием (72). Тогда справедливы равномерная $H^{1}$-оченка (73) для $\rho$ и C-стабилизачия для $\check{~}:$

$$
\left\|\check{\rho}(\cdot, t)-\bar{\rho}_{S}(\cdot)\right\|_{C(\bar{J})} \rightarrow 0 \quad n p u \quad t \rightarrow \infty .
$$

Если дополнительно выполнены условия (75), то справедливы также оцен$\kappa u(76) u(77)$.

Справедливость этих результатов следует из [10; теоремы 1,5 и утверждение 3], см. также [10; следствие 5]. Для вьвода п. 2) используется также утверждение 7 настоящей статьи, гарантирующее то, что $\omega$-предельное множество для $\check{\rho}$ сводится к единственной точке $\bar{\rho}_{S}$.

Теперь обратимся к основному результату параграфа.

ТЕОРЕМА 3. 1) Пусть выполнень условия пп. 1) и 2) утверждения 11 вместе с условиями (72). Тогда справедливы следующие оценки скорости стабилизачии при $t \geqslant t_{0}$ с достаточно больиим $t_{0}$ :

а) $L^{2}(J)$-оценка

$$
\check{\delta}_{0}(t):=\left\|\check{\rho}(\cdot, t)-\bar{\rho}_{S}(\cdot)\right\|_{L^{2}(J)}+\|\breve{v}(\cdot, t)\|_{L^{2}(J)} \leqslant \breve{d}_{0}\left(t, t_{0}\right) ;
$$

б) комбинированная оценка

$$
\check{\delta}_{1}(t):=\left\|\check{\rho}(\cdot, t)-\bar{\rho}_{S}(\cdot)\right\|_{H^{1}(J)}+\|\breve{v}(\cdot, t)\|_{L^{2}(J)} \leqslant \check{d}_{1}\left(t, t_{0}\right)
$$

в предположении, что

$$
p^{\prime \prime} \in L_{\mathrm{loc}}^{\infty}\left(\mathbb{R}^{+}\right), \quad\left\|\left(f_{S}\right)_{r}\right\|_{L^{2}\left(J ; C\left[r_{0}, \bar{R}\right]\right)} \leqslant C_{1}(N) ;
$$

в) $H^{1}(J)$-оченка

$$
\check{\delta}_{2}(t):=\left\|\check{\rho}(\cdot, t)-\bar{\rho}_{S}(\cdot)\right\|_{H^{1}(J)}+\|\check{v}(\cdot, t)\|_{H^{1}(J)} \leqslant \check{d}_{2}\left(t, t_{0}\right)
$$

в предположении, что справедливы условия (169) и (75). 
Здесь $\check{d}_{i}\left(t, t_{0}\right), i=0,1,2$, представляют собой модифицированнъе правъце части оценок (79)-(81) соответственно с $\check{\delta}_{i}\left(t_{0}\right)$ в роли $\delta_{i}\left(t_{0}\right)$, а также $c\|\Delta \check{f}[\check{r}](\cdot, \tau)\|_{L^{2}(J)}$ в роли $\|\Delta f[m](\cdot, \tau)\|_{L^{2}\left(\Omega_{\tau}\right)}$.

2) Пусть $\bar{\rho}_{S}$ - любое статически устойчивое стационарное решение (в отличие от условий на него в утверждении $11, n .2)$ ). Если данные удовлетворяют следующему условию близости к стационарньм:

$$
\left\|\check{\rho}^{0}-\bar{\rho}_{S}\right\|_{L^{2}(J)}+\left\|\check{v}^{0}\right\|_{L^{2}(J)}+\left\|\bar{f}_{1}\right\|_{L^{1}\left(\mathbb{R}^{+}\right)}+\left\|\bar{f}_{2}\right\|_{L^{2}\left(\mathbb{R}^{+}\right)}+\left\|\Delta p_{\Gamma}\right\|_{L^{2}\left(\mathbb{R}^{+}\right)} \leqslant \varepsilon_{0}
$$

с достаточно малым $\varepsilon_{0}>0$, то n. 1) справедлив при $t_{0}=0$, т.е. при любых $t \geqslant 0$, причем входящие в $\breve{d}_{i}\left(t, t_{0}\right)$ величинь $K$ и $a_{i}, i=0,1,2$, не зависят от данных; в этом смысле $\bar{\rho}_{S}$ динамически устойчиво.

Этот результат верен также при немонотонной функции состояния $p$ (опуская второе условие (10)) при условии, что значения $\bar{\rho}_{S}$ на $\bar{J}$ принадлежат какому-либо интервалу устойчивости р.

3) Пусть выполнены условия $n .1$ утверждения 11 вместе с $p^{\prime}>0,(72)$ u (141) ( с $C(\bar{J})$ суженныцм до $\left.H^{1}(J)\right)$. Тогда все подпункты а)-в) в п. 1) верны при любых $t \geqslant 0$ без условия близости (171) (кроме того, оценка (72) не требуется в подпункте а)). Дополнительно при сужении условия (162) до (22) все оценки в подпунктах справедливы равномерно относительно данных.

ДокАЗАТЕЛЬство. Шаг 1. Будем применять подход из [25], [24]. Фактически рассуждения близки к соответствуюшим в доказательстве теоремы 2. Возвратившись к лагранжеву закону сохранения энергии (161), сначала преобразуем член

$$
\frac{d}{d t} \check{\mathscr{F}}[\check{\rho}]=\int_{J}\left\{\left(p_{\Gamma, S}-\widehat{p}(\check{\eta})\right) D(\check{\varkappa} \check{v})-\check{h}[\check{V}] \check{\varkappa} \check{v}\right\} d \chi
$$

в силу уравнений (155) и (160). Воспользовавшись интегральным тождеством (126), найдем

$$
\frac{d}{d t} \check{\mathscr{F}}[\check{\rho}]=\int_{J}\left\{\left(\widehat{p}\left(\eta_{S}\right)-\widehat{p}(\check{\eta})\right) D(\check{\varkappa} \check{v})+\left(\check{h}\left[V_{S}\right]-\check{h}[\check{V}]\right) \check{\varkappa} \check{v}\right\} d \chi=\frac{d}{d t} \widetilde{\mathscr{U}}
$$

$\mathrm{c}$

$$
\widetilde{\mathscr{U}}=\widetilde{\mathscr{U}}\left[\check{\eta}, \eta_{S}\right]:=\int_{J}\left(\Pi\left(\check{\eta}, \eta_{S}\right)+\mathrm{H}\left[\check{V}, V_{S}\right]\right) d \chi
$$

и $\mathrm{H}\left[\check{V}, V_{S}\right](\chi, t)=\mathrm{H}\left(\check{V}(\chi, t), V_{S}(\chi), \chi\right)$,

$$
\Pi\left(\check{\eta}, \eta_{S}\right)=\int_{\eta_{S}}^{\check{\eta}}\left(\widehat{p}\left(\eta_{S}\right)-\widehat{p}(\zeta)\right) d \zeta, \quad \mathrm{H}\left(W, W_{S}, \chi\right):=\int_{W_{S}}^{W}\left(h\left(W_{S}, \chi\right)-h(\nu, \chi)\right) d \nu
$$

снова в силу уравнений (155) и (160).

Теперь из лагранжева закона сохранения энергии (161) выведем (ср. с (85))

$$
\frac{d}{d t}(\check{\mathscr{E}}+\widetilde{\mathscr{U}})+K^{-1}\|D(\check{\varkappa} \check{v})\|_{J}^{2} \leqslant K\left(\|\Delta \check{f}[\check{r}]\|_{J} \check{\mathscr{E}}^{1 / 2}+\left(\Delta p_{\Gamma}\right)^{2}\right)
$$

Заметим, что

$$
\begin{aligned}
\Pi\left(\check{\eta}, \eta_{S}\right)+\mathrm{H}\left[\check{V}, V_{S}\right]= & -\int_{0}^{1} \widehat{p}^{\prime}\left((1-\alpha) \eta_{S}+\alpha \check{\eta}\right)(1-\alpha) d \alpha\left(\check{\eta}-\eta_{S}\right)^{2} \\
& -\int_{0}^{1} \check{h}_{\nu}\left[(1-\alpha) V_{S}+\alpha \check{V}\right](1-\alpha) d \alpha\left(\check{V}-V_{S}\right)^{2}
\end{aligned}
$$


и

$$
\left\|\check{V}-V_{S}\right\|_{C(\bar{J})}=\left\|\int_{0}^{x}\left(\check{\eta}-\eta_{S}\right) d \xi\right\|_{C(\bar{J})} \leqslant \sqrt{M}\left\|\check{\eta}-\eta_{S}\right\|_{J} .
$$

Следовательно, в силу условий (10) и (134)

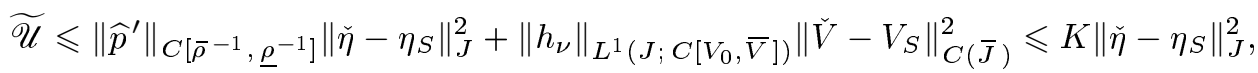

где $\bar{V}:=\bar{R}^{k+1} /(k+1)$, ср. с (88). С другой стороны, сначала непосредственной проверкой с учетом тождества (126), а затем применением свойства (139) при $V=V_{S}$ получим

$$
\widetilde{\mathscr{U}}\left[\check{\eta}, \eta_{S}\right]=\mathscr{P}[\check{V}]-\mathscr{P}\left[V_{S}\right] \geqslant K^{-1}\left\|\check{\eta}-\eta_{S}\right\|_{J}^{2}
$$

при достаточно больших $t \geqslant t_{1}$. Поэтому

$$
\check{\mathscr{E}}+\widetilde{\mathscr{U}} \asymp\|\check{v}\|_{J}^{2}+\left\|\check{\eta}-\eta_{S}\right\|_{J}^{2} .
$$

Шаг 2. Перепишем уравнение импульса (156) в виде

$$
\frac{1}{\check{\varkappa}} D_{t} \check{v}=D\left[\mu(\check{\rho}) \check{\rho} D(\check{\varkappa} \check{v})-\widehat{p}(\check{\eta})+\widehat{p}\left(\eta_{S}\right)\right]+\check{h}[\check{V}]-\check{h}\left[V_{S}\right]+\frac{1}{\check{\varkappa}} \Delta \check{f}[\check{r}]
$$

при помощи стационарного уравнения (120). Взяв $L^{2}(J)$-скалярное произведение с $\breve{V}-V_{S}$ и использовав граничные условия (158) и (121), найдем

$$
\begin{gathered}
\int_{J}\left\{\frac{1}{\check{\varkappa}}\left(D_{t} \check{v}\right)\left(\check{V}-V_{S}\right)+\left[\mu(\check{\rho}) \check{\rho} D(\check{\varkappa} \check{v})-\left(\widehat{p}(\check{\eta})-\widehat{p}\left(\eta_{S}\right)\right)\right]\left(\check{\eta}-\eta_{S}\right)\right\} d \chi \\
=\int_{J}\left(\check{h}[\check{V}]-\check{h}\left[V_{S}\right]+\frac{1}{\check{\varkappa}} \Delta \check{f}[\check{r}]\right)\left(\check{V}-V_{S}\right) d \chi \\
+\left.\left[\left(\widetilde{\mu}_{1}(\check{\rho}) \frac{\check{v}}{\check{r}}-\Delta p_{\Gamma}\right)\left(\check{V}-V_{S}\right)\right]\right|_{\chi=M} .
\end{gathered}
$$

Теперь применим формулы

$$
\begin{aligned}
& \frac{1}{\check{\varkappa}}\left(D_{t} \check{v}\right)\left(\check{V}-V_{S}\right)=D_{t}\left[\frac{\check{v}}{\check{\varkappa}}\left(\check{V}-V_{S}\right)\right]-\frac{\check{v}^{2}}{k+1}\left(1+k \frac{V_{S}}{\check{V}}\right), \\
& \mu(\check{\rho}) \check{\rho}(D(\check{\varkappa} \check{v}))\left(\check{\eta}-\eta_{S}\right)=\mu(\check{\rho})\left(1-\frac{\eta_{S}}{\check{\eta}}\right) D_{t} \check{\eta}=D_{t} \mathscr{M}\left(\check{\eta}, \eta_{S}\right)
\end{aligned}
$$

с $\mathscr{M}\left(\check{\eta}, \eta_{S}\right):=\int_{\eta_{S}}^{\check{\eta}} \mu\left(\frac{1}{\zeta}\right)\left(1-\frac{\eta_{S}}{\zeta}\right) d \zeta$, которые уже использовались в [25] и возникают в результате применения уравнений (155) и (160). Как следствие установим

$$
\begin{aligned}
\frac{d}{d t} \int_{J}\left(\mathscr{M}\left(\check{\eta}, \eta_{S}\right)+\frac{\check{v}}{\check{\varkappa}}\left(\check{V}-V_{S}\right)\right) d \chi+\widetilde{\mathscr{B}}\left(\check{\eta}, \eta_{S}\right) \\
=\widetilde{b}:=\int_{J}\left[\frac{\check{v}^{2}}{k+1}\left(1+k \frac{V_{S}}{\check{V}}\right)+\frac{1}{\check{\varkappa}} \Delta \check{f}[\check{r}]\left(\check{V}-V_{S}\right)\right] d \chi \\
+\left.\left[\left(\widetilde{\mu}_{1}(\check{\rho}) \frac{\check{\varkappa} \check{v}}{(k+1) \check{V}}-\Delta p_{\Gamma}\right)\left(\check{V}-V_{S}\right)\right]\right|_{\chi=M} ;
\end{aligned}
$$

напомним, что форма $\widetilde{\mathscr{B}}(\cdot, \cdot)$ была введена в $(125)$. 
Справедливы следующие соотношение и оценки:

$$
\begin{gathered}
\mathscr{M}\left(\check{\eta}, \eta_{S}\right) \asymp\left(\check{\eta}-\eta_{S}\right)^{2}, \\
\left|\int_{J} \frac{\check{\tilde{\varkappa}}}{\check{\mathscr{L}}}\left(\check{V}-V_{S}\right) d \chi\right| \leqslant K\|\check{v}\|_{J}\left\|\check{\eta}-\eta_{S}\right\|_{J}, \\
\widetilde{\mathscr{B}}\left(\check{\eta}, \eta_{S}\right) \geqslant \mathscr{J}\left[\check{V}-V_{S}\right]+o\left(\left\|\check{\eta}-\eta_{S}\right\|_{J}^{2}\right) \geqslant K^{-1}\left\|\check{\eta}-\eta_{S}\right\|_{J}^{2}
\end{gathered}
$$

при достаточно больших $t \geqslant t_{2}$ и

$$
\begin{aligned}
|\widetilde{b}| & \leqslant K\left(\|\check{v}\|_{J}^{2}+\|\Delta \check{f}[\check{r}]\|_{J}\left\|\check{V}-V_{S}\right\|_{J}+\left(\|\check{\varkappa} \check{v}\|_{C(\bar{J})}+\left|\Delta p_{\Gamma}\right|\right)\left\|\check{V}-V_{S}\right\|_{C(\bar{J})}\right) \\
& \leqslant \varepsilon\left\|\check{\eta}-\eta_{S}\right\|_{J}^{2}+K_{1}\left(\varepsilon^{-1}\|\check{\varkappa} \check{v}\|_{C(\bar{J})}^{2}+\|\Delta \check{f}[\check{r}]\|_{J}\left\|\check{\eta}-\eta_{S}\right\|_{J}+\left(\Delta p_{\Gamma}\right)^{2}\right)
\end{aligned}
$$

при любых $\varepsilon \in(0,1]$. Следовательно, равенство (177) влечет неравенство

$$
\begin{aligned}
\frac{d}{d t} \int_{J} & \left(\mathscr{M}\left(\check{\eta}, \eta_{S}\right)+\frac{\check{v}}{\check{\varkappa}}\left(\check{V}-V_{S}\right)\right) d \chi+K^{-1}\left\|\check{\eta}-\eta_{S}\right\|_{J}^{2} \\
& \leqslant K\left(\|\check{\varkappa} \check{v}\|_{C(\bar{J})}^{2}+\|\Delta \check{f}[\check{r}]\|_{J}\left\|\check{\eta}-\eta_{S}\right\|_{J}+\left(\Delta p_{\Gamma}\right)^{2}\right) .
\end{aligned}
$$

Скомбинировав его с неравенством (173) и применив соотношения (176), (178) и (179), найдем

$$
\frac{d}{d t} \widetilde{\mathscr{V}}_{0}+K^{-1}\left(\|D(\check{\varkappa} \check{v})\|_{J}^{2}+\left\|\check{\eta}-\eta_{S}\right\|_{J}^{2}\right) \leqslant K\left(\|\Delta \check{f}[\check{r}]\|_{J} \widetilde{\mathscr{V}}_{0}^{1 / 2}+\left(\Delta p_{\Gamma}\right)^{2}\right)
$$

вместе с

$$
\widetilde{\mathscr{V}}_{0}:=\widetilde{\mathscr{E}}+\widetilde{\mathscr{U}}+\varepsilon_{0} \int_{J}\left(\mathscr{M}\left(\check{\eta}, \eta_{S}\right)+\frac{\check{v}}{\check{\varkappa}}\left(\check{V}-V_{S}\right)\right) d \chi \asymp\|\check{v}\|_{J}^{2}+\left\|\check{\eta}-\eta_{S}\right\|_{J}^{2}
$$

при достаточно малом $\varepsilon_{0}:=1 / K$. Поэтому $\widetilde{\mathscr{V}}_{0}$ является функционалом Ляпунова и справедлива оценка (167).

Шаг 3. Чтобы доказать оценки (168) и (170), несколько модифицируем рассуждения из [25], [24]. Сначала преобразуем уравнение импульса (156) с учетом уравнений $(155),(160)$ и (118) следующим образом:

$$
D_{t}\left[\frac{\check{v}}{\check{\varkappa}}+D\left(\widehat{A}(\check{\eta})-\widehat{A}\left(\eta_{S}\right)\right)\right]+D\left(\widehat{p}(\check{\eta})-\widehat{p}\left(\eta_{S}\right)\right)=\frac{\check{f}[\check{r}]}{\check{\varkappa}}-\check{h}\left[V_{S}\right]-\frac{k}{k+1} \frac{\check{v}^{2}}{\check{V}^{2}} .
$$

Положив $\Delta \omega(\check{\eta})=\omega(\check{\eta})-\omega\left(\eta_{S}\right)$ для $\omega=\widehat{A}, \widehat{p}$ и т.д. и взяв $L^{2}(J)$-скалярное произведение последнего уравнения с $\check{v} / \check{\varkappa}+D \Delta \widehat{A}(\check{\eta})$, получим

$$
\begin{aligned}
\frac{d}{d t} \widetilde{\mathscr{Y}}+ & \int_{J}(D \Delta \check{p}(\check{\eta})) D \Delta \widehat{A}(\check{\eta}) d \chi=-\int_{J}(D \Delta \check{p}(\check{\eta})) \frac{\check{\varkappa}}{\check{\varkappa}} d \chi \\
& +\int_{J}\left(\check{h}[\check{V}]-\check{h}\left[V_{S}\right]+\frac{\Delta \check{f}[\check{r}]}{\check{\varkappa}}-\frac{k}{k+1} \frac{\check{v}^{2}}{\check{V}}\right)\left(\frac{\check{v}}{\check{\varkappa}}+D \Delta \widehat{A}(\check{\eta})\right) d \chi,
\end{aligned}
$$

где $\widetilde{\mathscr{Y}}:=\frac{1}{2} \int_{J}\left(\frac{\check{v}}{\check{\varkappa}}+D \Delta \widehat{A}(\check{\eta})\right)^{2} d \chi$. 
Далее, воспользуемся формулой $D \Delta \omega(\check{\eta})=\omega^{\prime}(\check{\eta}) D \Delta \check{\eta}+\left(\Delta \omega^{\prime}\right)(\check{\eta}) D \eta_{S}$ для $\omega=\widehat{A}, \widehat{p}$, применим условия (169) вместе с оценками

$$
\begin{aligned}
& \sup _{t \geqslant 0}\|D \check{\eta}\|_{J} \leqslant K, \quad\left\|D \eta_{S}\right\|_{L^{\infty}(J)} \leqslant K, \\
& \left\|\check{v}^{2}\right\|_{J} \leqslant\|\check{v}\|_{J}\|\check{v}\|_{L^{\infty}(J)} \leqslant K\|D(\check{\varkappa} \check{v})\|_{J}
\end{aligned}
$$

(которые следуют из оценок (73), (43) и (71) соответственно). Поэтому (воспользовавшись также неравенством Коши с $\varepsilon$ ) найдем

$$
\frac{d}{d t} \widetilde{\mathscr{Y}}+K^{-1}\|D \Delta \check{\eta}\|_{J}^{2} \leqslant K\left[\|\Delta \check{\eta}\|_{J}^{2}+\|D(\check{\varkappa} \check{v})\|_{J}^{2}+\|\Delta \check{f}[\check{r}]\|_{J}\left(\|\check{v}\|_{J}+\|\Delta \check{\eta}\|_{H^{1}(J)}\right)\right]
$$

вместе с

$$
K^{-1}\|D \Delta \check{\eta}\|_{J}^{2}-K\left(\|\Delta \check{\eta}\|_{J}^{2}+\|\check{v}\|_{J}^{2}\right) \leqslant \widetilde{\mathscr{Y}} \leqslant K\left(\|\Delta \check{\eta}\|_{H^{1}(J)}^{2}+\|\check{v}\|_{J}^{2}\right) .
$$

Скомбинировав (181) с (183) и применив (182) и (184), получим

$$
\frac{d}{d t} \widetilde{\mathscr{V}}_{1}+K^{-1}\left(\|D(\check{\varkappa} \check{v})\|_{J}^{2}+\left\|\check{\eta}-\eta_{S}\right\|_{H^{1}(J)}^{2}\right) \leqslant K\left(\|\Delta \check{f}[\check{r}]\|_{J} \widetilde{\mathscr{V}}_{1}^{1 / 2}+\left(\Delta p_{\Gamma}\right)^{2}\right)
$$

вместе с

$$
\widetilde{\mathscr{V}}_{1}:=\widetilde{\mathscr{V}}_{0}+\varepsilon_{1} \widetilde{\mathscr{Y}} \asymp\|\check{v}\|_{J}^{2}+\left\|\check{\eta}-\eta_{S}\right\|_{H^{1}(J)}^{2}
$$

при достаточно малом $\varepsilon_{1}:=K^{-1}$. Поэтому $\widetilde{\mathscr{V}}_{1}$ является функционалом Ляпунова и справедлива оценка (168).

Шаг 4. Преобразуем теперь уравнение импульса (156) к виду

$$
D_{t}(\check{\varkappa} \check{v})-k \check{r}^{k-1} \breve{v}^{2}=\check{\varkappa}^{2} D\left\{\mu(\check{\rho}) \check{\rho} D(\check{\varkappa} \check{v})-\widehat{p}(\check{\eta})+\widehat{p}\left(\eta_{S}\right)\right\}+\check{\varkappa}\left(\check{f}[\check{r}]-\check{\varkappa} \check{h}\left[V_{S}\right]\right) .
$$

Далее положим $\check{\sigma}:=\mu(\check{\rho}) \check{\rho} D(\check{\varkappa} \check{v})-\widetilde{\mu}_{1}(\check{\rho}) \check{v} / \check{r}-\widehat{p}(\check{\eta})$,

$$
\widetilde{\Phi}:=\frac{\check{\sigma}+\widehat{p}\left(\eta_{S}\right)+\Delta p_{\Gamma}}{\mu(\check{\rho}) \check{\rho}}=D(\check{\varkappa} \check{v})-\omega_{2}(\check{\rho}) \frac{\check{v}}{\check{r}}-\frac{\widehat{p}(\check{\eta})-\widehat{p}\left(\eta_{S}\right)-\Delta p_{\Gamma}}{\mu(\check{\rho}) \check{\rho}}
$$

(значит, $\left.\widetilde{\Phi}\right|_{\chi=M}=0$ ) и

$$
\widetilde{b}_{3}:=\check{\varkappa}\{D(\mu(\check{\rho}) \check{\rho})\} \widetilde{\Phi}+\frac{k}{\check{r}} \check{v}^{2}+\check{\varkappa} D\left(\widetilde{\mu}_{1}(\check{\rho}) \frac{\check{v}}{\check{r}}\right)+\check{\varkappa}\left(\check{h}[\check{V}]-\check{h}\left[V_{S}\right]\right)+\Delta \check{f}[\check{r}] .
$$

Это позволяет переписать уравнение (187) следующим образом:

$$
D_{t}(\check{\varkappa} \check{v})=\check{\varkappa}^{2} \mu(\check{\rho}) \check{\rho} D \widetilde{\Phi}+\check{\varkappa} \widetilde{b}_{3} .
$$

Последнее уравнение влечет равенство (ср. с (106))

$$
\begin{aligned}
& \frac{1}{2} \frac{d}{d t} \int_{J} \widetilde{\Phi}^{2} d \chi+\int_{J} \check{\varkappa}^{2} \mu(\check{\rho}) \check{\rho}(D \widetilde{\Phi})^{2} d \chi \\
& \quad=-\int_{J}\left\{\left(D_{t}\left(\omega_{2}(\check{\rho}) \frac{\check{v}}{\check{r}}\right)+D_{t} \frac{\widehat{p}(\check{\eta})-\widehat{p}\left(\eta_{S}\right)-\Delta p_{\Gamma}}{\mu(\check{\rho}) \check{\rho}}\right) \widetilde{\Phi}+\check{\varkappa} \widetilde{\iota}_{3} D \widetilde{\Phi}\right\} d \chi .
\end{aligned}
$$


Подобно (107) (с учетом (155) и (188)) имеем

$$
\left|D_{t}\left(\omega_{2}(\check{\rho}) \frac{\check{v}}{\check{r}}\right)\right| \leqslant K\left(|D(\check{\varkappa} \check{v})||\check{v}|+\left|\check{\varkappa} \mu(\check{\rho}) \check{\rho} D \widetilde{\Phi}+\widetilde{b}_{3}-(k+1) \frac{\check{v}^{2}}{\check{r}}\right|\right),
$$

кроме того,

$$
\left|D_{t} \frac{\widehat{p}(\check{\eta})-\widehat{p}\left(\eta_{S}\right)-\Delta p_{\Gamma}}{\mu(\check{\rho}) \check{\rho}}\right| \leqslant K\left(|D(\check{\varkappa} \check{\imath})|+\left|p_{\Gamma}^{\prime}\right|\right) .
$$

Применив оценку $\|\check{v}\|_{C_{b}\left(\bar{Q}_{L}\right)} \leqslant K($ см. (76)), получим

$$
\begin{aligned}
\frac{d}{d t} \int_{J} \widetilde{\Phi}^{2} d \chi+K^{-1}\|D \widetilde{\Phi}\|_{J}^{2} \\
\quad \leqslant K\left\{\left(\|D(\check{\varkappa} \check{v})\|_{J}+\|D \widetilde{\Phi}\|_{J}+\left|p_{\Gamma}^{\prime}\right|\right)\|\widetilde{\Phi}\|_{J}+\left(\left\|\widetilde{b}_{3}\right\|_{J}+\|\check{v}\|_{J}\right)\|D \widetilde{\Phi}\|_{J}\right\} .
\end{aligned}
$$

По аналогии с (108) и (105) справедливы следующие оценки:

$$
\begin{aligned}
\left\|\widetilde{b}_{3}\right\|_{J} \leqslant & \varepsilon\|D \widetilde{\Phi}\|_{J}+K\left(\varepsilon^{-1}\|\widetilde{\Phi}\|_{J}+\|D(\check{\varkappa} \check{v})\|_{J}\right. \\
& \left.+\left\|\check{h}_{\nu}\right\|_{L^{2}\left(J ; C\left[V_{0}, \bar{V}\right]\right)}\left\|\check{V}-V_{S}\right\|_{C(\bar{J})}+\|\Delta \check{f}[\check{r}]\|_{J}\right)
\end{aligned}
$$

при любом $\varepsilon>0$ и

$$
\begin{aligned}
\|D(\check{\varkappa} \check{v})\|_{J} & -K\left(\|\check{v}\|_{J}+\left\|\check{\eta}-\eta_{S}\right\|_{J}+\left|\Delta p_{\Gamma}\right|\right) \\
& \leqslant\|\widetilde{\Phi}\|_{J} \leqslant K\left(\|D(\check{\varkappa} \check{v})\|_{J}+\left\|\check{\eta}-\eta_{S}\right\|_{J}+\left|\Delta p_{\Gamma}\right|\right) .
\end{aligned}
$$

Следовательно, (190) приводит к неравенствам

$$
\begin{aligned}
\frac{d}{d t} \int_{J} & \widetilde{\Phi}^{2} d \chi+K^{-1}\|D \widetilde{\Phi}\|_{J}^{2} \\
& \leqslant K\left(\|\widetilde{\Phi}\|_{J}^{2}+\|D(\check{\varkappa} \check{v})\|_{J}^{2}+\left|p_{\Gamma}^{\prime}\right|\|\widetilde{\Phi}\|_{J}+\left\|\check{V}-V_{S}\right\|_{C(\bar{J})}^{2}+\|\Delta \check{f}[\check{r}]\|_{J}^{2}\right) \\
& \leqslant K_{1}\left(\|D(\check{\varkappa} \check{v})\|_{J}^{2}+\left\|\check{\eta}-\eta_{S}\right\|_{J}^{2}+\|\Delta \check{f}[\check{r}]\|_{J}^{2}+\left(\Delta p_{\Gamma}\right)^{2}+\left|p_{\Gamma}^{\prime}\right|\|\widetilde{\Phi}\|_{J}\right) .
\end{aligned}
$$

Скомбинировав результат с соотношениями (185) и (110) и воспользовавшись (186) и (191), выведем

$$
\begin{gathered}
\frac{d}{d t} \widetilde{\mathscr{V}}_{2}+K^{-1}\left(\|D(\check{\varkappa} \check{v})\|_{J}^{2}+\left\|\check{\eta}-\eta_{S}\right\|_{H^{1}(J)}^{2}+\left(\Delta p_{\Gamma}\right)^{2}+\|D \widetilde{\Phi}\|_{J}^{2}\right) \\
\leqslant K\left(\|\Delta \check{f}[\check{r}]\|_{J}^{2}+\left(\Delta p_{\Gamma}\right)^{2}+\left|p_{\Gamma}^{\prime}\right| \widetilde{\mathscr{V}}_{2}^{1 / 2}\right)
\end{gathered}
$$

вместе с

$$
\widetilde{\mathscr{V}}_{2}:=\widetilde{\mathscr{V}}_{1}+\varepsilon_{2}\|\widetilde{\Phi}\|_{J}^{2}+\left(\Delta p_{\Gamma}\right)^{2} \asymp\|\check{v}\|_{H^{1}(J)}^{2}+\left\|\check{\eta}-\eta_{S}\right\|_{H^{1}(J)}^{2}+\left(\Delta p_{\Gamma}\right)^{2}
$$

при достаточно малом $\varepsilon_{2}:=K^{-1}$. Таким образом, последняя из оценок в п. 1), а именно (170), также справедлива. Пункт 1) доказан.

Шаг 5. Пункт 2) доказывается аналогично и немного проше, чем соответствующий п. 2) в теореме 2.

В случае, когда выполнены условия п. 3), оценка снизу (175) верна при всех $t \geqslant 0$, см. неравенство (142). Промежуточное соотношение в оценке снизу (180) может быть опушено в силу условия (141), и эта оценка также выполняется при любых $t \geqslant 0$. Поскольку теперь нет необходимости в свойстве стабилизации (166), условие (72) и оценка (73) не требуются для доказательства оценки (167). В заключение напомним, что при вьполнении условия (22) имеем $\underline{\rho}=K^{-1}$ (см. [10; теорема 1, п. 3]) и, таким образом, величины $K$ и $K_{i}$ в оценках скорости стабилизации не зависят от данных. 
ЗАмечание 10. Неравенство (174) позволяет добавить слагаемое $\left|R(t)-R_{S}\right|$ в левую часть оценки (167). Кроме того, дополнительно к этой оценке имеем

$$
\widetilde{\delta}_{0}(t):=\breve{\delta}_{0}(t)+\left|\check{\rho}(M, t)-\bar{\rho}_{S}(M)\right| \leqslant \widetilde{d}_{0}\left(t, t_{0}\right),
$$

где $\widetilde{d}_{0}\left(t, t_{0}\right)$ отличается от $\check{d}_{0}\left(t, t_{0}\right)$ слагаемым $\widetilde{\delta}_{0}\left(t_{0}\right)$ в роли $\check{\delta}_{0}\left(t_{0}\right)$. Эта оценка возникает в результате комбинирования (181) и (93), а также применения равенств $\left.\left(\check{\rho}-\bar{\rho}_{S}\right)\right|_{\chi=M}=\rho_{R}-\rho_{\Gamma, S}$ и $\|v\|_{C\left(\bar{\Omega}_{t}\right)}=\|\check{v}\|_{C(\bar{J})}$.

УТВЕРЖДЕНИЕ 12. Пусть верны равномерные оценки $\rho \leqslant \rho(\cdot, t) \leqslant K$. Тогда ясно, что $\|v(\cdot, t)\|_{H^{j}\left(\Omega_{t}\right)} \asymp\|\check{v}(\cdot, t)\|_{H^{j}(J)}$ при $j=0 \overline{1}$, где, например, $H^{0}(J)=L^{2}(J)$. Более того, если $R(t) \leqslant R_{S}+\varepsilon_{S}$, то тажже

$$
\left\|\rho(\cdot, t)-\rho_{S}(\cdot)\right\|_{H^{j}\left(\Omega_{t}\right)} \asymp\left\|\check{\rho}(\cdot, t)-\bar{\rho}_{S}(\cdot)\right\|_{H^{j}(J)} \quad \text { npu } \quad j=0,1
$$

при условии, что в случае $j=1$ выполняются (17).

ДоКАЗАТЕЛЬСТВО. Положим $\check{\rho}_{S}:=\rho_{S}(\check{r})$. Заметим, что

$$
\begin{gathered}
\left\|\check{\rho}_{S}-\bar{\rho}_{S}\right\|_{C(\bar{J})} \leqslant\left\|\left(\rho_{S}\right)_{r}\right\|_{C\left[r_{0}, R_{S}+\varepsilon_{S}\right]} \Delta \\
\Delta:=\left\|\check{r}-r_{S}\right\|_{C(\bar{J})} \leqslant K\left\|\check{V}-V_{S}\right\|_{C(\bar{J})} \leqslant K_{1}\left\|\check{\rho}-\bar{\rho}_{S}\right\|_{J},
\end{gathered}
$$

см. (174). Более того, $D\left(\check{\rho}_{S}-\bar{\rho}_{S}\right)=\left(\left(\rho_{S}\right)_{r}(\check{r})-\left(\rho_{S}\right)_{r}\left(r_{S}\right)\right) D \check{r}+\left(\rho_{S}\right)_{r}\left(r_{S}\right) D\left(\check{r}-r_{S}\right)$ и

$$
\left|D\left(\check{r}-r_{S}\right)\right|=\left|\frac{1}{\check{\rho} \check{r}^{k}}-\frac{1}{\bar{\rho}_{S} r_{S}^{k}}\right| \leqslant K\left(\left|\check{r}-r_{S}\right|+\left|\check{\rho}-\bar{\rho}_{S}\right|\right) .
$$

При вьполнении условий (17) имеем

$$
\left|\left(\rho_{S}\right)_{r}(\check{r})-\left(\rho_{S}\right)_{r}\left(r_{S}\right)\right| \leqslant \int_{\max \left\{\check{r}-\Delta, r_{0}\right\}}^{\min \left\{\check{r}+\Delta, R_{S}+\varepsilon_{S}\right\}}\left|\left(\rho_{S}\right)_{r r}(q)\right| d q
$$

поэтому

$$
\begin{aligned}
\left\|D\left(\check{\rho}_{S}-\bar{\rho}_{S}\right)\right\|_{J} \leqslant & 2 \Delta\left\|\left(\rho_{S}\right)_{r r}\right\|_{\left(r_{0}, R_{S}+\varepsilon_{S}\right)}\|D \check{r}\|_{L^{\infty}(J)} \\
& +\left\|\left(\rho_{S}\right)_{r}\right\|_{C\left[r_{0}, R_{S}+\varepsilon_{S}\right]}\left\|D\left(\check{r}-r_{S}\right)\right\|_{J} \\
\leqslant & K\left(\Delta+\left\|\check{\rho}-\bar{\rho}_{S}\right\|_{J} \leqslant K_{1}\left\|\check{\rho}-\bar{\rho}_{S}\right\|_{J} .\right.
\end{aligned}
$$

Поскольку $\left\|\rho-\rho_{S}\right\|_{H^{j}\left(\Omega_{t}\right)} \asymp\left\|\check{\rho}-\check{\rho}_{S}\right\|_{H^{j}(J)}$, то по неравенству треугольника

$$
\left\|\rho-\rho_{S}\right\|_{H^{j}\left(\Omega_{t}\right)} \leqslant K\left\|\check{\rho}-\bar{\rho}_{S}\right\|_{H^{j}(J)}
$$

при $j=0,1$.

С другой стороны, аналогичньм образом можно установить оценки

$$
\begin{aligned}
\left\|\bar{\rho}_{S}(m)-\bar{\rho}_{S}\left(m_{S}\right)\right\|_{C\left(\bar{\Omega}_{t}\right)} \leqslant & \left\|D \bar{\rho}_{S}\right\|_{C(\bar{J})}\left\|m-m_{S}\right\|_{C\left(\bar{\Omega}_{t}\right)} \leqslant K\left\|\rho-\rho_{S}\right\|_{\Omega_{t}}, \\
\left\|\left(\bar{\rho}_{S}(m)-\bar{\rho}_{S}\left(m_{S}\right)\right)_{r}\right\|_{\Omega_{t}} \leqslant & \left\|D^{2} \bar{\rho}_{S}\right\|_{J}\left\|m-m_{S}\right\|_{C\left(\bar{\Omega}_{t}\right)}\left\|m_{r}\right\|_{C\left(\bar{\Omega}_{t}\right)} \\
& +\left\|D \bar{\rho}_{S}\right\|_{C(\bar{J})}\left\|m-m_{S}\right\|_{\Omega_{t}} \leqslant K\left\|\rho-\rho_{S}\right\|_{\Omega_{t}} .
\end{aligned}
$$

Поскольку $\left\|\check{\rho}-\bar{\rho}_{S}\right\|_{H^{j}(J)} \asymp\left\|\rho-\bar{\rho}_{S}(m)\right\|_{H^{j}\left(\Omega_{t}\right)}$ и $\bar{\rho}_{S}\left(m_{S}\right)=\rho_{S}$, то получим $\left\|\check{\rho}-\bar{\rho}_{S}\right\|_{H^{j}(J)} \leqslant K\left\|\rho-\rho_{S}\right\|_{H^{j}\left(\Omega_{t}\right)}$ при $j=0,1$, что завершает доказательство. 
Результат п. 2) в теореме 3 в случае немонотонной функции $p$ улучшает соответствуюший ему в [35] (устранением априорного условия на $p(\rho)$ ). Результат п. 3 ) восходит к работам [18], [17], [24]. Замечание 10 и утверждение 12 позволяют установить непосредственную связь между оценками скорости стабилизации в эйлеровых координатах в теореме 2 и соответствуюших им в лагранжевых координатах в теореме 3.

Сушественная часть этой работы была вьполнена в течение визита А. А. Злотника осенњю 2003 г. в Отделение теоретической и прикладной физики Комиссариата по атомной энергии Франции в Иль де Франс (г. Брюйер-ле-Шатель), которому он признателен за гостеприимство.

\section{Список литературы}

1. Чандрасекхар C. Введение в учение о строении звезд. М.: ИЛ, 1950.

2. Ledoux P. Stellar stability // Handbuch der Physik. V. 51. Berlin: Springer-Verlag, 1958. P. 605-688.

3. Ledoux P. Stellar stability // Stars and stellar structure / ed. L.H. Aller et al. Chicago: Univ. Chicago Press, 1965. P. 499-574.

4. Chiu H.- Y. Stellar physics. V. I. Blaisdell: Waltham, 1968.

5. Huntley J. M., Saslaw W. C. The distribution of stars in galactic nuclei: loaded polytropes // Astrophys. J. 1975. V. 199. P. 328-335.

6. Kimura H. A study of simple polytropes. Parts I, II // Publ. Astron. Soc. Japan. 1981. V. 33. P. 273-298; 299-312.

7. Ducomet B. Hydrodynamical models of gaseous stars // Rev. Math. Phys. 1996. V. 8. P. $957-1000$.

8. Lin S. S. Stability of gaseous stars in spherically symmetric motions // SIAM J. Math. Anal. 1997. V. 28. P. 539-569.

9. Heinzle J. M., Uggla C. Newtonian stellar models // Ann. Physics. 2003. V. 308. № 1. P. $18-61$.

10. Ducomet B., Zlotnik A. Viscous compressible barotropic symmetric flows with free boundary under general mass force. Part I: Uniform-in-time bounds and stabilization // Math. Methods Appl. Sci. 2005. V. 28. P. 827-863.

11. Злотник A. А., Дюкоме Б. Глобальное поведение симметричных течений вязкой сжимаемой баротропной жидкости со свободной границей для общей массовой силы // Докл. PAH. 2004. T. 398. № 4. C. 444-448.

12. Kuan W. C., Lin S. S. Numbers of equilibria for the equation of self-gravitating isentropic gas surrounding a solid ball // Japan J. Indust. Appl. Math. 1996. V. 13. P. 311-331.

13. Fu C.C., Lin S. S. On the critical mass of the collapse of a gaseous star in spherically symmetric and isentropic motion // Japan J. Indust. Appl. Math. 1998. V. 15. P. 461-469.

14. Strömer G., Zajaczkowski $W$. On the existence and properties of the rotationally symmetric equilibrium states of compressible barotropic self-gravitating fluids // Indiana Univ. Math. J. 1997. V. 46. P. 1181-1220.

15. Злотник А. А., Дюкоме Б. Задача симметричного равновесия сжимаемой баротропной жидкости со свободной границей для общей массовой силы // Докл. РАН. 2005. Т. 401. № 2. C. 154-159.

16. Злотник A.А., Дюкоме Б. Функционалы Ляпунова симметричных течений вязкой сжимаемой баротропной жидкости со свободной границей для общей массовой силы // Докл. РАН. 2005. Т. 402. №1. С. 14-19.

17. Злотник А. А., Нгуен ЖК Бао. Свойства и асимптотическое поведение решений одной задачи одномерного движения вязкого баротропного газа // Матем. заметки. 1994. Т. 55. № 5. C. 51-68.

18. Злотник A.A. Об уравнениях одномерного движения вязкого баротропного газа при наличии массовой силы // Сиб. матем. журн. 1992. Т. 33. № 5. С. 62-79.

19. Mucha P. Compressible Navier-Stokes system in 1-D // Math. Methods Appl. Sci. 2001. V. 21. P. $607-622$. 
20. Matsumura A., Yanagi S. Uniform boundedness of the solutions for a one-dimensional isentropic model system of a compressible viscous gas // Comm. Math. Phys. 1996. V. 175. P. 259-274.

21. Straškraba I., Zlotnik A. Global behavior of 1d-viscous compressible barotropic fluid with a free boundary and large data // J. Math. Fluid Mech. 2003. V. 5. P. 119-143; Erratum 2004. V. 6. P. 249-250.

22. Zlotnik A. Global behaviour of 1-D viscous compressible barotropic flows with free boundary and self-gravitation // Math. Methods Appl. Sci. 2003. V. 26. P. 671-690.

23. Злотник A. А. Об одной задаче Нишида // ЖВМиМФ. 1998. Т. 38. № 8. С. 1279-1286.

24. Злотник A. А., Нгуен Жа Бао. Глобальные свойства симметричных решений задачи движения вязкого баротропного газа со свободной границей // Вестн. МЭИ. 1998. Т. 5. №6. C. 52-61.

25. Злотник A. A. Равномерные оценки и стабилизация симметричных решений одной квазилинейной системы уравнений // Дифференц. уравнения. 2000. Т. 36. № 5. С. 634-646.

26. Zlotnik A. A. Global properties of a difference approximation of the free boundary problem for a symmetric motion of a viscous barotropic gas // Russian J. Numer. Anal. Math. Modelling. 1999. V. 14. P. 527-548.

27. Matsumura A. Large-time behavior of the spherically symmetric solutions of an isothermal model of compressible viscous gas // Transp. Theory Statist. Phys. 1992. V. 21. P. 579-592.

28. Yanagi $S$. Asymptotic stability of the spherically symmetric solutions for an isentropic model of compressible viscous gas // Japan J. Indust. Appl. Math. 1997. V. 14. P. 215-243.

29. Matušu-Nečasova S̆., Okada M., Makino T. Free boundary problem for the equation of spherically symmetric motion of a viscous gas. III // Japan J. Indust. Appl. Math. 1997. V. 14. P. 199-213.

30. Ducomet B. Some asymptotics for a reactive Navier-Stokes-Poisson system // Math. Models Methods. Appl. Sci. 1999. V. 9. P. 1039-1076.

31. Хартман П. Обыкновенные дифференциальные уравнения. М.: Мир, 1970.

32. Penel P., Straškraba I. Global behavior of compressible fluid with a free boundary and large data // Applied nonlinear analysis / ed. A. Sequeira et al. New York: Kluwer Academic, 1999. P. 427-442.

33. Zlotnik A. A., Maksimov M. V. On symmetric equilibrium of an isothermal gas with free boundary and mass force // Abstr. Appl. Anal. 2006 (to appear).

34. Ладыженская О. А., Солонников В. А., Уральцева Н. Н. Линейныеи квазилинейные уравнения параболического типа. М.: Наука, 1967.

35. Ducomet B., Zlotnik A. Remark on the stabilization of a viscous barotropic medium with a nonmonotone equation of state // Appl. Math. Lett. 2001. V. 14. P. 921-928.

Московский энергетический институт;

Département de Physique Théorique et Appliquée,

CEA/DAM Ile de France, France

E-mail: zlotnik@apmsun.mpei.ac.ru

bernard.ducomet@cea.fr
Поступила в редакцию 25.06 .2004 и 25.04 .2005 